\title{
Injective tests of low complexity in the plane
}

\author{
Dominique LECOMTE and Rafael ZAMORA ${ }^{1}$
}

July 3, 2021

- Université Paris 6, Institut de Mathématiques de Jussieu, Projet Analyse Fonctionnelle Couloir 16-26, 4ème étage, Case 247, 4, place Jussieu, 75252 Paris Cedex 05, France dominique.lecomte@upmc.fr

Université de Picardie, I.U.T. de l'Oise, site de Creil, 13, allée de la faïencerie, 60107 Creil, France

-1 Université Paris 6, Institut de Mathématiques de Jussieu, Projet Analyse Fonctionnelle Couloir 15-16, 5ème étage, Case 247, 4, place Jussieu, 75252 Paris Cedex 05, France rafael.zamora@imj-prg.fr

\begin{abstract}
We study injective versions of the characterization of sets potentially in a Wadge class of Borel sets, for the first Borel and Lavrentieff classes. We also study the case of oriented graphs in terms of continuous homomorphisms, injective or not.
\end{abstract}

2010 Mathematics Subject Classification. Primary: 03E15, Secondary: 26A21, 54H05

Keywords and phrases. acyclic, Borel, class, dichotomy, difference, homomorphism, injective, locally countable, oriented graph, reduction, Wadge 


\section{Introduction}

The reader should see $[\mathrm{K}]$ for the standard descriptive set theoretic notation used in this paper. This work is a contribution to the study of analytic subsets of the plane. We are looking for results of the following form: either a situation is simple, or it is more complicated than a situation in a collection of known complicated situations. The notion of complexity we consider is the following, and defined in [Lo3].

Definition 1.1 (Louveau) Let $X, Y$ be Polish spaces, $B$ be a Borel subset of $X \times Y$, and $\Gamma$ be a class of Borel sets closed under continuous pre-images. We say that $B$ is potentially in $\Gamma$ (denoted $B \in \operatorname{pot}(\boldsymbol{\Gamma}))$ if there are finer Polish topologies $\sigma$ and $\tau$ on $X$ and $Y$, respectively, such that $B$, viewed as a subset of the product $(X, \sigma) \times(Y, \tau)$, is in $\boldsymbol{\Gamma}$.

The quasi-order $\leq_{B}$ of Borel reducibility was intensively considered in the study of analytic equivalence relations during the last decades. The notion of potential complexity is a natural invariant for $\leq_{B}$ : if $E \leq_{B} F$ and $F \in \operatorname{pot}(\boldsymbol{\Gamma})$, then $E \in \operatorname{pot}(\boldsymbol{\Gamma})$ too. However, as shown in [L1]-[L6] and [L8], $\leq_{B}$ is not the right notion of comparison to study potential complexity, in the general context, because of cycle problems. A good notion of comparison is as follows. Let $X, Y, X^{\prime}, Y^{\prime}$ be topological spaces and $A, B \subseteq X \times Y, A^{\prime}, B^{\prime} \subseteq X^{\prime} \times Y^{\prime}$. We write

$$
\begin{aligned}
(X, Y, A, B) \leq & \left(X^{\prime}, Y^{\prime}, A^{\prime}, B^{\prime}\right) \Leftrightarrow \\
& \exists f: X \rightarrow X^{\prime} \exists g: Y \rightarrow Y^{\prime} \text { continuous with } A \subseteq(f \times g)^{-1}\left(A^{\prime}\right) \text { and } B \subseteq(f \times g)^{-1}\left(B^{\prime}\right) .
\end{aligned}
$$

Our motivating result is the following (see [L8]).

Definition 1.2 We say that a class $\boldsymbol{\Gamma}$ of subsets of zero-dimensional Polish spaces is a Wadge class of Borel sets if there is a Borel subset $\mathbf{A}$ of $\omega^{\omega}$ such that for any zero-dimensional Polish space $X$, and for any $A \subseteq X, A$ is in $\boldsymbol{\Gamma}$ if and only if there is $f: X \rightarrow \omega^{\omega}$ continuous such that $A=f^{-1}(\mathbf{A})$. In this case, we say that $\mathbf{A}$ is $\mathbf{\Gamma}$-complete.

If $\boldsymbol{\Gamma}$ is a class of sets, then $\check{\Gamma}:=\{\neg A \mid A \in \boldsymbol{\Gamma}\}$ is the dual class of $\boldsymbol{\Gamma}$, and $\boldsymbol{\Gamma}$ is self-dual if $\boldsymbol{\Gamma}=\check{\boldsymbol{\Gamma}}$. We set $\Delta(\boldsymbol{\Gamma}):=\boldsymbol{\Gamma} \cap \check{\Gamma}$.

Theorem 1.3 (Lecomte) Let $\boldsymbol{\Gamma}$ be a Wadge class of Borel sets, or the class $\boldsymbol{\Delta}_{\xi}^{0}$ for some countable ordinal $\xi \geq 1$. Then there are concrete disjoint Borel relations $\mathbb{S}_{0}, \mathbb{S}_{1}$ on $2^{\omega}$ such that, for any Polish spaces $X, Y$, and for any disjoint analytic subsets $A, B$ of $X \times Y$, exactly one of the following holds:

(a) the set $A$ is separable from $B$ by a pot $(\boldsymbol{\Gamma})$ set,

(b) $\left(2^{\omega}, 2^{\omega}, \mathbb{S}_{0}, \mathbb{S}_{1}\right) \leq(X, Y, A, B)$.

It is natural to ask whether we can have $f$ and $g$ injective if (b) holds. Debs proved that this is the case if $\boldsymbol{\Gamma}$ is a non self-dual Borel class of rank at least three (i.e., a class $\boldsymbol{\Sigma}_{\xi}^{0}$ or $\boldsymbol{\Pi}_{\xi}^{0}$ with $\xi \geq 3$ ). As mentioned in [L8], there is also an injectivity result for the non self-dual Wadge classes of Borel sets of level at least three. Some results in [L4] and [L8] show that we cannot have $f$ and $g$ injective if (b) holds and $\Gamma$ is a non self-dual Borel class of rank one or two, or the class of clopen sets, because of cycle problems again. 
The work of Kechris, Solecki and Todorčević indicates a way to try to solve this problem. Let us recall one of their results in this direction. All the relations considered in this paper will be binary.

Definition 1.4 Let $X$ be a set, and $A$ be a relation on $X$.

(a) $\Delta(X):=\left\{(x, y) \in X^{2} \mid x=y\right\}$ is the diagonal of $X$.

(b) We say that $A$ is irreflexive if $A$ does not meet $\Delta(X)$.

(c) $A^{-1}:=\left\{(x, y) \in X^{2} \mid(y, x) \in A\right\}$, and $s(A):=A \cup A^{-1}$ is the symmetrization of $A$.

(d) We say that $A$ is symmetric if $A=A^{-1}$.

(e) We say that $A$ is a graph if $A$ is irreflexive and symmetric.

(f) We say that $A$ is acyclic if there is no injective sequence $\left(x_{i}\right)_{i \leq n}$ of points of $X$ with $n \geq 2$, $\left(x_{i}, x_{i+1}\right) \in A$ for each $i<n$, and $\left(x_{n}, x_{0}\right) \in A$.

(g) We say that $A$ is locally countable if $A$ has countable horizontal and vertical sections (this also makes sense in a rectangular product $X \times Y$ ).

Notation. Let $\left(s_{n}\right)_{n \in \omega}$ be a sequence of finite binary sequences with the following properties:

(a) $\left(s_{n}\right)_{n \in \omega}$ is dense in $2^{<\omega}$. This means that for each $s \in 2^{<\omega}$, there is $n \in \omega$ such that $s_{n}$ extends $s$ (denoted $s \subseteq s_{n}$ ).

(b) $\left|s_{n}\right|=n$.

We put $\mathbb{G}_{0}:=\left\{\left(s_{n} 0 \gamma, s_{n} 1 \gamma\right) \mid n \in \omega \wedge \gamma \in 2^{\omega}\right\}$. The following result is proved in [K-S-T].

Theorem 1.5 (Kechris, Solecki, Todorčević) Let $X$ be a Polish space, and A be an analytic graph on $X$. We assume that $A$ is acyclic or locally countable. Then exactly one of the following holds:

(a) there is $c: X \rightarrow \omega$ Borel such that $A \subseteq(c \times c)^{-1}(\neg \Delta(\omega))$,

(b) there is $f: 2^{\omega} \rightarrow X$ injective continuous such that $s\left(\mathbb{G}_{0}\right) \subseteq(f \times f)^{-1}(A)$.

This seems to indicate that there is a hope to get $f$ and $g$ injective in Theorem 1.3 (b) for the first classes of the hierarchy if we assume acyclicity or local countability. This is the main purpose of this paper, and leads to the following notation. Let $X, Y, X^{\prime}, Y^{\prime}$ be topological spaces and $A, B \subseteq X \times Y$, $A^{\prime}, B^{\prime} \subseteq X^{\prime} \times Y^{\prime}$. We write

$$
\begin{aligned}
&(X, Y, A, B) \sqsubseteq\left(X^{\prime}, Y^{\prime}, A^{\prime}, B^{\prime}\right) \Leftrightarrow \\
& \quad \exists f: X \rightarrow X^{\prime} \quad \exists g: Y \rightarrow Y^{\prime} \text { injective continuous with } A \subseteq(f \times g)^{-1}\left(A^{\prime}\right) \text { and } B \subseteq(f \times g)^{-1}\left(B^{\prime}\right) .
\end{aligned}
$$

We want to study the Borel and Wadge classes of the locally countable Borel relations: the Borel classes of rank one or two, the Lavrentieff classes built with the open sets (the classes of differences of open sets), their dual classes and their ambiguous classes. We will also study the Lavrentieff classes built with the $F_{\sigma}$ sets and their dual classes.

Definition 1.6 Let $\eta<\omega_{1}$. If $\left(O_{\theta}\right)_{\theta<\eta}$ is an increasing sequence of subsets of a set $X$, then

$$
D\left(\left(O_{\theta}\right)_{\theta<\eta}\right):=\left\{x \in X \mid \exists \theta<\eta \text { parity }(\theta) \neq \operatorname{parity}(\eta) \text { and } x \in O_{\theta} \backslash\left(\bigcup_{\theta^{\prime}<\theta} O_{\theta^{\prime}}\right)\right\} .
$$

Now $D_{\eta}\left(\boldsymbol{\Sigma}_{\xi}^{0}\right)(X):=\left\{D\left(\left(O_{\theta}\right)_{\theta<\eta}\right) \mid \forall \theta<\eta \quad O_{\theta} \in \boldsymbol{\Sigma}_{\xi}^{0}(X)\right\}$, for each $1 \leq \xi<\omega_{1}$. The classes $D_{\eta}\left(\boldsymbol{\Sigma}_{\xi}^{0}\right), \check{D}_{\eta}\left(\boldsymbol{\Sigma}_{\xi}^{0}\right)$ and $\Delta\left(D_{\eta}\left(\boldsymbol{\Sigma}_{\xi}^{0}\right)\right)$ form the difference hierarchy. 
Some recent work of the first author shows that having $f$ and $g$ injective in Theorem 1.3 (b) can be used to get results of reduction on the whole product, under some acyclicity and also topological assumptions. Some of the results in the present paper will be used by the first author in a future article on this topic. This work is also motivated by the work of Louveau on oriented graphs in [Lo4].

Definition 1.7 Let $X$ be a set, and $A$ be a relation on $X$.

(a) We say that $A$ is antisymmetric if $A \cap A^{-1} \subseteq \Delta(X)$.

(b) We say that $A$ is an oriented graph if $A$ is irreflexive and antisymmetric.

It follows from results of Wadge and Martin that inclusion well-orders

$\{\boldsymbol{\Gamma} \cup \check{\boldsymbol{\Gamma}} \mid \boldsymbol{\Gamma}$ Wadge class of Borel sets $\}$,

giving rise to an ordinal assignment $w(\boldsymbol{\Gamma})$. If $G$ is an analytic oriented graph, then we can define $w(G)$ as the least $w(\boldsymbol{\Gamma})$ such that $G$ is separable from $G^{-1}$ by a $\operatorname{pot}(\boldsymbol{\Gamma})$ set $C$. It is well defined by the separation theorem. Moreover, it is useless in the definition to distinguish between dual classes, for if $C$ separates $G$ from $G^{-1}$, then so does $\neg C^{-1}$, which is potentially in $\check{\Gamma}$. The main property of this assignment is that $w(G) \leq w(H)$ if there is a Borel homomorphism from $G$ into $H$. Louveau also considers a rough approximation of $w(G)$, which is the least countable ordinal $\xi$ for which $G$ is separable from $G^{-1}$ by a $\operatorname{pot}\left(\Delta_{\xi}^{0}\right)$ set. He proves the following.

Theorem 1.8 (Louveau) Let $\xi \in\{1,2\}$. Then there is a concrete analytic oriented graph $\mathbb{G}_{\xi}$ on $2^{\omega}$ such that, for any Polish space $X$, and for any analytic oriented graph $G$ on $X$, exactly one of the following holds:

(a) the set $G$ is separable from $G^{-1}$ by a pot $\left(\boldsymbol{\Delta}_{\xi}^{0}\right)$ set,

(b) there is $f: 2^{\omega} \rightarrow X$ continuous such that $\mathbb{G}_{\xi} \subseteq(f \times f)^{-1}(G)$.

Our main results are the following.

- We generalize Theorem 1.8 to all the $\Delta_{\xi}^{0}$, s, and all the Wadge classes of Borel sets.

Theorem 1.9 Let $\boldsymbol{\Gamma}$ be a Wadge class of Borel sets, or the class $\boldsymbol{\Delta}_{\xi}^{0}$ for some countable ordinal $\xi \geq 1$. Then there is a concrete Borel oriented graph $\mathbb{G}_{\Gamma}$ on $2^{\omega}$ such that, for any Polish space $X$, and for any analytic oriented graph $G$ on $X$, exactly one of the following holds:

(a) the set $G$ is separable from $G^{-1}$ by a pot $(\boldsymbol{\Gamma})$ set,

(b) there is $f: 2^{\omega} \rightarrow X$ continuous such that $\mathbb{G}_{\Gamma} \subseteq(f \times f)^{-1}(G)$.

We also investigate the injective version of this, for the first classes of the hierarchies again.

- In the sequel, it will be very convenient to say that a relation $A$ on a set $X$ is s-acyclic if $s(A)$ is acyclic.

Theorem 1.10 Let $\boldsymbol{\Gamma} \in\left\{D_{\eta}\left(\boldsymbol{\Sigma}_{1}^{0}\right), \check{D}_{\eta}\left(\boldsymbol{\Sigma}_{1}^{0}\right), D_{n}\left(\boldsymbol{\Sigma}_{2}^{0}\right), \check{D}_{n}\left(\boldsymbol{\Sigma}_{2}^{0}\right) \mid 1 \leq \eta<\omega_{1}, 1 \leq n<\omega\right\} \cup\left\{\boldsymbol{\Delta}_{2}^{0}\right\}$. Then there are concrete disjoint Borel relations $\mathbb{S}_{0}, \mathbb{S}_{1}$ on $2^{\omega}$ such that, for any Polish space $X$, and for any disjoint analytic relations $A, B$ on $X$ with s-acyclic union, exactly one of the following holds:

(a) the set $A$ is separable from $B$ by a pot $(\boldsymbol{\Gamma})$ set,

(b) $\left(2^{\omega}, 2^{\omega}, \mathbb{S}_{0}, \mathbb{S}_{1}\right) \sqsubseteq(X, Y, A, B)$. 
In fact, we prove a number of extensions of this result. It also holds

- for $\eta=0$ if we replace $2^{\omega}$ with 1 ,

- with $f=g$ if $\boldsymbol{\Gamma} \notin\left\{D_{\eta}\left(\boldsymbol{\Sigma}_{1}^{0}\right), \check{D}_{\eta}\left(\boldsymbol{\Sigma}_{1}^{0}\right) \mid \eta<\omega_{1}\right\}$; if $\boldsymbol{\Gamma} \in\left\{D_{\eta}\left(\boldsymbol{\Sigma}_{1}^{0}\right), \check{D}_{\eta}\left(\boldsymbol{\Sigma}_{1}^{0}\right) \mid \eta<\omega_{1}\right\}$, then there is an antichain basis with two elements for the square reduction (it is rather unusual to have an antichain basis but no minimum object in this kind of dichotomy),

- if we assume that $A \cup B$ is locally countable instead of s-acyclic when $\Gamma \subseteq \Pi_{2}^{0}$ (this also holds in rectangular products $X \times Y$ ),

- if we only assume that $A$ is s-acyclic or locally countable when $\boldsymbol{\Gamma}=\mathbf{\Pi}_{2}^{0}$.

The situation is more complicated for the ambiguous classes.

Theorem 1.11 Let $\boldsymbol{\Gamma} \in\left\{\Delta\left(D_{\eta}\left(\boldsymbol{\Sigma}_{1}^{0}\right)\right) \mid 1 \leq \eta<\omega_{1}\right\}$. Then there is a concrete finite antichain $\mathcal{A}$, made of tuples $\left(2^{\omega}, 2^{\omega}, \mathbb{S}_{0}, \mathbb{S}_{1}\right)$ where $\mathbb{S}_{0}$, $\mathbb{S}_{1}$ are disjoint Borel relations $\mathbb{S}_{0}$, $\mathbb{S}_{1}$ on $2^{\omega}$, such that, for any Polish space $X$, and for any disjoint analytic relations $A, B$ on $X$ whose union is contained in a potentially closed s-acyclic relation $R$, exactly one of the following holds:

(a) the set $A$ is separable from $B$ by a pot $(\boldsymbol{\Gamma})$ set,

(b) there is $\left(2^{\omega}, 2^{\omega}, \mathbb{A}, \mathbb{B}\right) \in \mathcal{A}$ with $\left(2^{\omega}, 2^{\omega}, \mathbb{A}, \mathbb{B}\right) \sqsubseteq(X, Y, A, B)$.

Here again, we can say more. This also holds

- if we assume that $R$ is locally countable instead of s-acyclic (this also holds in rectangular products $X \times Y)$,

- in all those cases, $\mathcal{A}$ has size three if $\eta$ is a successor ordinal, and size one if $\eta$ is a limit ordinal (it is quite remarkable that the situation depends on the fact that $\eta$ is limit or not, it confirms the difference observed in the description of Wadge classes of Borel sets in terms of operations on sets present in [Lo1]),

- with $f=g$, but in order to ensure this $\mathcal{A}$ must have size six if $\eta$ is a successor ordinal, and size two if $\eta$ is a limit ordinal.

- We characterize when part (b) in the injective reduction property holds.

Theorem 1.12 Let $\boldsymbol{\Gamma} \in\left\{D_{\eta}\left(\boldsymbol{\Sigma}_{1}^{0}\right), \check{D}_{\eta}\left(\boldsymbol{\Sigma}_{1}^{0}\right), D_{n}\left(\boldsymbol{\Sigma}_{2}^{0}\right), \check{D}_{n}\left(\boldsymbol{\Sigma}_{2}^{0}\right) \mid 1 \leq \eta<\omega_{1}, 1 \leq n<\omega\right\} \cup\left\{\boldsymbol{\Delta}_{2}^{0}\right\}$. Then there are concrete disjoint Borel relations $\mathbb{S}_{0}, \mathbb{S}_{1}$ on $2^{\omega}$ such that, for any Polish space $X$, and for any disjoint analytic relations $A, B$ on $X$, the following are equivalent:

(1) there is an s-acyclic relation $R \in \boldsymbol{\Sigma}_{1}^{1}$ such that $A \cap R$ is not separable from $B \cap R$ by a pot $(\boldsymbol{\Gamma})$ set, (2) $\left(2^{\omega}, 2^{\omega}, \mathbb{S}_{0}, \mathbb{S}_{1}\right) \sqsubseteq(X, Y, A, B)$.

The same kind of extensions as before hold (except that we cannot assume local countability instead of s-acyclicity for the classes of rank two). 
Theorem 1.13 Let $\boldsymbol{\Gamma} \in\left\{\Delta\left(D_{\eta}\left(\boldsymbol{\Sigma}_{1}^{0}\right)\right) \mid 1 \leq \eta<\omega_{1}\right\}$. Then there is a concrete finite antichain $\mathcal{A}$, made of tuples $\left(2^{\omega}, 2^{\omega}, \mathbb{S}_{0}, \mathbb{S}_{1}\right)$ where $\mathbb{S}_{0}, \mathbb{S}_{1}$ are disjoint Borel relations $\mathbb{S}_{0}, \mathbb{S}_{1}$ on $2^{\omega}$, such that, for any Polish space $X$, and for any disjoint analytic relations $A, B$ on $X$, the following are equivalent: (1) there is a potentially closed s-acyclic relation $R \in \Sigma_{1}^{1}$ such that $A \cap R$ is not separable from $B \cap R$ by a $\operatorname{pot}(\boldsymbol{\Gamma})$ set,

(2) there is $\left(2^{\omega}, 2^{\omega}, \mathbb{A}, \mathbb{B}\right) \in \mathcal{A}$ with $\left(2^{\omega}, 2^{\omega}, \mathbb{A}, \mathbb{B}\right) \sqsubseteq(X, Y, A, B)$.

Here again, the same kind of extensions as before hold.

- The injective versions of Theorem 1.9 mentioned earlier are as follows.

Theorem 1.14 Let $\boldsymbol{\Gamma} \in\left\{D_{\eta}\left(\boldsymbol{\Sigma}_{1}^{0}\right), \check{D}_{\eta}\left(\boldsymbol{\Sigma}_{1}^{0}\right), D_{n}\left(\boldsymbol{\Sigma}_{2}^{0}\right), \check{D}_{n}\left(\boldsymbol{\Sigma}_{2}^{0}\right) \mid 1 \leq \eta<\omega_{1}, 1 \leq n<\omega\right\} \cup\left\{\boldsymbol{\Delta}_{2}^{0}\right\}$. Then there is a concrete Borel oriented graph $\mathbb{G}_{\Gamma}$ on $2^{\omega}$ such that, for any Polish space $X$, and for any analytic s-acyclic oriented graph $G$ on $X$, exactly one of the following holds:

(a) the set $G$ is separable from $G^{-1}$ by a pot $(\boldsymbol{\Gamma})$ set,

(b) there is $f: 2^{\omega} \rightarrow X$ injective continuous such that $\mathbb{G}_{\boldsymbol{\Gamma}} \subseteq(f \times f)^{-1}(G)$.

This result also holds if we assume that $G$ is locally countable instead of s-acyclic when $\mathbf{\Gamma} \subseteq \mathbf{\Pi}_{2}^{0}$.

Theorem 1.15 Let $\boldsymbol{\Gamma} \in\left\{\Delta\left(D_{\eta}\left(\boldsymbol{\Sigma}_{1}^{0}\right)\right) \mid 1 \leq \eta<\omega_{1}\right\}$. Then there is a concrete finite antichain $\mathcal{A}$, made of Borel oriented graphs on $2^{\omega}$, such that, for any Polish space $X$, and for any analytic oriented graph $G$ on $X$ contained in a potentially closed s-acyclic relation, exactly one of the following holds:

(a) the set $G$ is separable from $G^{-1}$ by a pot $(\boldsymbol{\Gamma})$ set,

(b) we can find $\mathbb{G}_{\boldsymbol{\Gamma}} \in \mathcal{A}$ and $f: 2^{\omega} \rightarrow X$ injective continuous such that $\mathbb{G}_{\boldsymbol{\Gamma}} \subseteq(f \times f)^{-1}(G)$.

The same kind of extensions as before hold, except that $\mathcal{A}$ has size three if $\eta$ is a successor ordinal, and size two if $\eta$ is a limit ordinal.

- At the end of the paper, we study the limits of our results and give negative results.

\section{Generalities}

\section{The acyclic and the locally countable cases}

In [K-S-T], Section 6, the authors introduce the notion of an almost acyclic analytic graph, in order to prove an injective version of the $\mathbb{G}_{0}$-dichotomy for acyclic or locally countable analytic graphs. We now give a similar definition, in order to prove injective versions of Theorem 1.3 for the first classes of the hierarchies. This definition is sufficient to cover all our cases, even if it is not always optimal.

Definition 2.1 Let $X$ be a Polish space, and $A$ be a relation on $X$. We say that $A$ is quasi-acyclic if there is a sequence $\left(C_{n}\right)_{n \in \omega}$ of pot $\left(\boldsymbol{\Pi}_{1}^{0}\right)$ relations on $X$ with disjoint union $A$ such that, for any $s(A)$ path $\left(z_{i}\right)_{i \leq 2}$ with $z_{0} \neq z_{2}$, and for any $n_{1}, \ldots, n_{k} \in \omega, C_{n_{i}}^{\prime} \in\left\{C_{n_{i}}, C_{n_{i}}^{-1}\right\}(1 \leq i \leq k), x_{1}, y_{1}, \ldots, x_{k}, y_{k}$ in $X \backslash\left\{z_{i} \mid i \leq 2\right\}$, if $\left(z_{0}, x_{1}\right),\left(z_{2}, y_{1}\right) \in C_{n_{1}}^{\prime},\left(x_{1}, x_{2}\right),\left(y_{1}, y_{2}\right) \in C_{n_{2}}^{\prime}, \ldots,\left(x_{k-1}, x_{k}\right),\left(y_{k-1}, y_{k}\right) \in C_{n_{k}}^{\prime}$ all hold, then $x_{k} \neq y_{k}$. 
Lemma 2.2 Let $X$ be a Polish space, and $A$ be a Borel relation on $X$. We assume that $A$ is either s-acyclic and pot $\left(\Sigma_{2}^{0}\right)$, or locally countable. Then $A$ is quasi-acyclic.

Proof. Assume first that $A$ is s-acyclic and $\operatorname{pot}\left(\boldsymbol{\Sigma}_{2}^{0}\right)$. Then we can write $A=\bigcup_{n \in \omega} C_{n}$, where $\left(C_{n}\right)_{n \in \omega}$ is a disjoint sequence of potentially closed relations on $X$. The acyclicity of $s(A)$ shows that $A$ is quasi-acyclic.

Assume now that $A$ is locally countable. By 18.10 in $[\mathrm{K}], A$ can be written as $\bigcup_{q \in \omega} G_{q}$, where $G_{q}$ is the Borel graph of a partial function $f_{q}$, and we may assume that the $G_{q}$ 's are pairwise disjoint. By 18.12 in [K], the projections of the $G_{q}$ 's are Borel. By Lemma 2.4.(a) in [L2], there is, for each $q$, a countable partition $\left(D_{p}^{q}\right)_{p \in \omega}$ of the domain of $f_{q}$ into Borel sets on which $f_{q}$ is injective. So the $C_{n}$ 's are the $\operatorname{Gr}\left(f_{q \mid D_{p}^{q}}\right)$ 's.

\section{$\underline{\text { Topologies }}$}

Let $Z$ be a recursively presented Polish space (see $[\mathrm{M}]$ for the basic notions of effective theory).

(1) The topology $\Delta_{Z}$ on $Z$ is generated by $\Delta_{1}^{1}(Z)$. This topology is Polish (see (iii) $\Rightarrow$ (i) in the proof of Theorem 3.4 in [Lo3]). The topology $\tau_{1}$ on $Z^{2}$ is $\Delta_{Z}^{2}$. If $2 \leq \xi<\omega_{1}^{\mathrm{CK}}$, then the topology $\tau_{\xi}$ on $Z^{2}$ is generated by $\Sigma_{1}^{1} \cap \boldsymbol{\Pi}_{<\xi}^{0}\left(\tau_{1}\right)$.

(2) The Gandy-Harrington topology on $Z$ is generated by $\Sigma_{1}^{1}(Z)$ and denoted $\Sigma_{Z}$. Recall the following facts about $\Sigma_{Z}$ (see [L7]).

(a) $\Sigma_{Z}$ is finer than the initial topology of $Z$.

(b) We set $\Omega_{Z}:=\left\{z \in Z \mid \omega_{1}^{z}=\omega_{1}^{\mathrm{CK}}\right\}$. Then $\Omega_{Z}$ is $\Sigma_{1}^{1}(Z)$ and dense in $\left(Z, \Sigma_{Z}\right)$.

(c) $W \cap \Omega_{Z}$ is a clopen subset of $\left(\Omega_{Z}, \Sigma_{Z}\right)$ for each $W \in \Sigma_{1}^{1}(Z)$.

(d) $\left(\Omega_{Z}, \Sigma_{Z}\right)$ is a zero-dimensional Polish space.

\section{The classes $D_{\eta}\left(\Sigma_{1}^{0}\right)$ and $\check{D}_{\eta}\left(\Sigma_{1}^{0}\right)$}

\section{Examples}

In Theorem 1.3, either $\mathbb{S}_{0}$ or $\mathbb{S}_{1}$ is not locally countable if $\boldsymbol{\Gamma}$ is not self-dual. If $\boldsymbol{\Gamma} \subseteq \boldsymbol{\Delta}_{2}^{0}$, we can find disjoint analytic locally countable relations $A, B$ on $2^{\omega}$ such that $A$ is not separable from $B$ by a $\operatorname{pot}(\boldsymbol{\Gamma})$ set, as we will see. This shows that, in order to get partial reductions with injectivity, we have to use examples different from those in [L8], so that we prove the following.

Notation. We introduce examples in the style of $\mathbb{G}_{0}$ in order to prove a dichotomy for the classes $D_{\eta}\left(\Sigma_{1}^{0}\right)$, where $\eta \geq 1$ is a countable ordinal.

- If $t \in 2^{<\omega}$, then $N_{t}:=\left\{\alpha \in 2^{\omega} \mid t \subseteq \alpha\right\}$ is the usual basic clopen set. 
- As in Section 2 in [L2] we inductively define $\varphi_{\eta}: \omega^{<\omega} \rightarrow\{-1\} \cup(\eta+1)$ by $\varphi_{\eta}(\emptyset)=\eta$ and

$$
\varphi_{\eta}(s n)=\left\{\begin{array}{l}
-1 \text { if } \varphi_{\eta}(s) \leq 0, \\
\theta \text { if } \varphi_{\eta}(s)=\theta+1, \\
\text { an odd ordinal such that the sequence }\left(\varphi_{\eta}(s n)\right)_{n \in \omega} \text { is cofinal in } \varphi_{\eta}(s) \\
\text { and strictly increasing if } \varphi_{\eta}(s)>0 \text { is limit. }
\end{array}\right.
$$

If no confusion is possible, then we will write $\varphi$ instead of $\varphi_{\eta}$. We set $T_{\eta}:=\left\{s \in \omega^{<\omega} \mid \varphi_{\eta}(s) \neq-1\right\}$, which is a wellfounded tree.

- Let $\left(p_{q}\right)_{q \in \omega}$ be the sequence of prime numbers, and $\left\langle.>_{\eta}: T_{\eta} \rightarrow \omega\right.$ be the following bijection. We define $I: T_{\eta} \rightarrow \omega$ by $I(\emptyset):=0$ and $I(s):=p_{0}^{s(0)+1} \ldots p_{|s|-1}^{s(|s|-1)+1}$ if $s \neq \emptyset$. As $I$ is injective, there is an increasing bijection $J: I\left[T_{\eta}\right] \rightarrow \omega$. We set $\left\langle.>_{\eta}:=J \circ I\right.$. Note that $\left\langle s q>_{\eta}-<s>_{\eta} \geq q+1\right.$ if $s q \in T_{\eta}$. Indeed, $I(s 0), \ldots, I(s(q-1))$ are strictly between $I(s)$ and $I(s q)$.

- Let $\psi: \omega \rightarrow 2^{<\omega}$ be the map defined by $\emptyset, \emptyset, 0,0,1,1,0^{2}, 0^{2}, 01,01,10,10,1^{2}, 1^{2}, \ldots$, so that $|\psi(q)| \leq q$ and $\psi[\{2 n \mid n \in \omega\}], \psi[\{2 n+1 \mid n \in \omega\}]=2^{<\omega}$.

- For each $s \in T_{\eta}$, we define $\left(t_{s}^{0}, t_{s}^{1}\right) \in(2 \times 2)^{<\omega}$ by $t_{\emptyset}^{\varepsilon}=\emptyset$, and $t_{s q}^{\varepsilon}=t_{s}^{\varepsilon} \psi(q) 0^{<s q>_{\eta}-<s>_{\eta}-|\psi(q)|-1_{\varepsilon}} \varepsilon$. Note that this is well defined, $\left|t_{s}^{\varepsilon}\right|=<s>_{\eta}$ and $\operatorname{Card}\left(\left\{l<<s>_{\eta} \mid t_{s}^{0}(l) \neq t_{s}^{1}(l)\right\}\right)=|s|$ for each $s \in T_{\eta}$.

- We set $\mathcal{T}^{\eta}:=\left\{\left(t_{s}^{0} w, t_{s}^{1} w\right) \mid s \in T_{\eta} \wedge w \in 2^{<\omega}\right\}$. The following properties are satisfied.

- $\mathcal{T}^{\eta}$ is a tree on $2 \times 2$, and $\left\lceil\mathcal{T}^{\eta}\right\rceil \subseteq \mathbb{E}_{0}:=\left\{(\alpha, \beta) \in 2^{\omega} \times 2^{\omega} \mid \exists m \in \omega \forall n>m \quad \alpha(n)=\beta(n)\right\}$ is locally countable.

- If $(s, t) \in \mathcal{T}^{\eta}$ and $s(l) \neq t(l)$, then $s(l)<t(l)$.

- For each $l \in \omega$, there is exactly one sequence $(u, v) \in \mathcal{T}^{\eta} \cap\left(2^{l+1} \times 2^{l+1}\right)$ such that $u(l) \neq v(l)$ since $t_{s q}^{0}\left(<s q>_{\eta}-1\right) \neq t_{s q}^{1}\left(<s q>_{\eta}-1\right)$ (in fact, $(u, v)$ is of the form $\left(t_{s}^{0}, t_{s}^{1}\right)$ for some $\left.s\right)$. In particular, $s\left(\mathcal{T}^{\eta} \cap\left(2^{l+1} \times 2^{l+1}\right)\right) \backslash \Delta\left(2^{l+1}\right)$ is a connected acyclic graph on $2^{l+1}$, inductively.

- We set, for $\varepsilon \in 2$,

$$
\mathbb{N}_{\varepsilon}^{\eta}:=\left\{\left(t_{s}^{0} \gamma, t_{s}^{1} \gamma\right) \mid s \in T_{\eta} \wedge \text { parity }(|s|)=\varepsilon \wedge \gamma \in 2^{\omega}\right\} .
$$

If $s \in T_{\eta}$, then $f_{s}: N_{t_{s}^{0}} \rightarrow N_{t_{s}^{1}}$ is the partial homeomophism with clopen domain and range defined by $f_{s}\left(t_{s}^{0} \gamma\right):=t_{s}^{1} \gamma$, so that $\mathbb{N}_{\varepsilon}^{\eta}=\bigcup_{s \in T_{\eta}, \text { parity }(|s|)=\varepsilon} \operatorname{Gr}\left(f_{s}\right)$. We set $C_{s}:=\bigcup_{q \in \omega} \operatorname{Gr}\left(f_{s q}\right)$ when it makes sense (i.e., when $\varphi_{\eta}(s) \geq 1$ ). For $\eta=0$, we set $\mathbb{N}_{0}^{\eta}:=1^{2}$ and $\mathbb{N}_{1}^{\eta}:=\emptyset\left(\right.$ in $1^{2}$ ).

Lemma 3.1 Let $\eta$ be a countable ordinal, and $C$ be a nonempty clopen subset of $2^{\omega}$.

(a) If $\varphi_{\eta}(s) \geq 1$ and $G$ is a dense $G_{\delta}$ subset of $2^{\omega}$, then $\overline{C_{s}} \cap(C \cap G)^{2} \subseteq \overline{C_{s} \cap(C \cap G)^{2}}$.

(b) $\mathbb{N}_{0}^{\eta} \cap C^{2}$ is not separable from $\mathbb{N}_{1}^{\eta} \cap C^{2}$ by a pot $\left(D_{\eta}\left(\Sigma_{1}^{0}\right)\right)$ set. 
Proof. (a) It is enough to prove that if $q \in \omega$, then $\operatorname{Gr}\left(f_{s q}\right) \cap C^{2} \subseteq \overline{\operatorname{Gr}\left(f_{s q}\right) \cap(C \cap G)^{2}}$. This comes from the proof of Lemma 3.5 in [L1], but we recall it for self-containedness. Let $U, V$ be open subsets of $C$ such that $\operatorname{Gr}\left(f_{s q}\right) \cap(U \times V) \neq \emptyset$. Then $N_{t_{s q}^{1}} \cap V \cap G$ is a dense $G_{\delta}$ subset of $N_{t_{s q}^{1}} \cap V$, so that $f_{s q}^{-1}(V \cap G)$ is a dense $G_{\delta}$ subset of $f_{s q}^{-1}(V)$. Thus $G \cap f_{s q}^{-1}(V)$ and $G \cap f_{s q}^{-1}(V \cap G)$ are dense $G_{\delta}$ subsets of $f_{s q}^{-1}(V)$. This gives $\alpha$ in this last set and $U \cap f_{s q}^{-1}(V)$. Therefore $\left(\alpha, f_{s q}(\alpha)\right)$ is in $\operatorname{Gr}\left(f_{s q}\right) \cap(C \cap G)^{2} \cap(U \times V)$.

(b) We may assume that $\eta \geq 1$. We argue by contradiction, which gives $P \in \operatorname{pot}\left(D_{\eta}\left(\Sigma_{1}^{0}\right)\right)$, and a dense $G_{\delta}$ subset of $2^{\omega}$ such that $P \cap G^{2} \in D_{\eta}\left(\Sigma_{1}^{0}\right)\left(G^{2}\right)$. So let $\left(O_{\theta}\right)_{\theta<\eta}$ be a sequence of open relations on

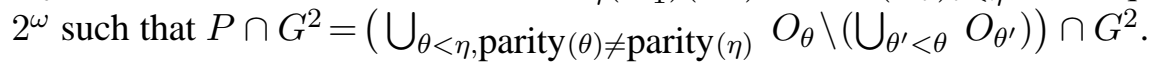

- Let us show that if $\theta \leq \eta, s \in T_{\eta}$ and $\varphi(s)=\theta$, then $\operatorname{Gr}\left(f_{s}\right) \cap(C \cap G)^{2} \subseteq \neg O_{\theta}$ if $\theta<\eta$, and $\operatorname{Gr}\left(f_{s}\right) \cap(C \cap G)^{2}$ is disjoint from $\bigcup_{\theta^{\prime}<\theta} O_{\theta^{\prime}}$ if $\theta=\eta$. The objects $s=\emptyset$ and $\theta=\eta$ will give the contradiction.

- We argue by induction on $\theta$. Note that if $s \in T_{\eta},|s|$ is even if and only if $\varphi(s)$ has the same parity as $\eta$. If $\theta=0$, then $|s|$ has the same parity as $\eta$, thus $\operatorname{Gr}\left(f_{s}\right) \cap(C \cap G)^{2} \subseteq \mathbb{N}_{\text {parity }(\eta)}^{\eta} \cap G^{2} \subseteq \neg O_{0}$.

- Assume that the result has been proved for $\theta^{\prime}<\theta$. If $\theta$ is the successor of $\theta^{\prime}$, then the induction assumption implies that $\operatorname{Gr}\left(f_{s q}\right) \cap(C \cap G)^{2} \subseteq \neg O_{\theta^{\prime}}$ for each q. So $C_{s} \cap(C \cap G)^{2} \subseteq \neg O_{\theta^{\prime}}$ and $\overline{C_{s} \cap(C \cap G)^{2}} \subseteq \neg O_{\theta^{\prime}}$. By (a), we get $\overline{C_{s}} \cap(C \cap G)^{2} \subseteq \overline{C_{s} \cap(C \cap G)^{2}}$, which gives the desired inclusion if $\theta=\eta$ since $\operatorname{Gr}\left(f_{s}\right) \subseteq \overline{C_{s}}$.

If $\theta<\eta$ and $|s|$ is even, then $\varphi(s)$ has the same parity as $\eta$ and the parity of $\theta^{\prime}$ is opposite to that of

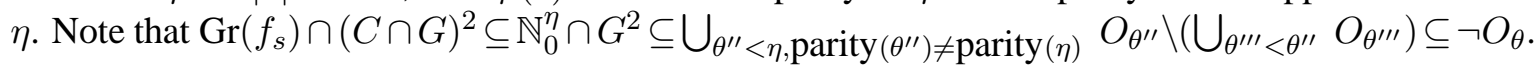

If $|s|$ is odd, then the parity of $\varphi(s)$ is opposite to that of $\eta$ and $\theta^{\prime}$ has the same parity as $\eta$. But if $s \in T_{\eta}$ has odd length, then

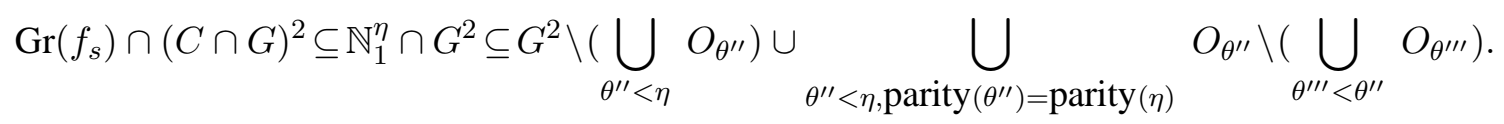

This gives the result.

- If $\theta$ is limit, then $(\varphi(s n))_{n \in \omega}$ is cofinal in $\varphi(s)$, and $\operatorname{Gr}\left(f_{s n}\right) \cap(C \cap G)^{2} \subseteq \neg O_{\varphi(s n)}$ by the induction assumption. If $\theta_{0}<\varphi(s)$, then there is $n\left(\theta_{0}\right)$ such that $\varphi(s n)>\theta_{0}$ if $n \geq n\left(\theta_{0}\right)$. Thus $\operatorname{Gr}\left(f_{s n}\right) \cap(C \cap G)^{2} \subseteq \neg O_{\theta_{0}}$ as soon as $n \geq n\left(\theta_{0}\right)$. But

$\operatorname{Gr}\left(f_{s}\right) \cap(C \cap G)^{2} \subseteq(C \cap G)^{2} \cap \overline{C_{s}} \backslash C_{s}=\overline{C_{s} \cap(C \cap G)^{2}} \backslash C_{s} \subseteq \overline{\bigcup_{n \geq n\left(\theta_{0}\right)} \operatorname{Gr}\left(f_{s n}\right) \cap(C \cap G)^{2}} \subseteq \neg O_{\theta_{0}}$.

Thus $\operatorname{Gr}\left(f_{s}\right) \cap(C \cap G)^{2} \subseteq \neg\left(\bigcup_{\theta^{\prime}<\theta} O_{\theta^{\prime}}\right)$.

If $\theta<\eta$, as $|s|$ has the same parity as $\eta$, we get $\operatorname{Gr}\left(f_{s}\right) \cap(C \cap G)^{2} \subseteq \mathbb{N}_{\text {parity }(\eta)}^{\eta} \cap G^{2}$, so that $\operatorname{Gr}\left(f_{s}\right) \cap(C \cap G)^{2} \subseteq \neg O_{\theta}$. 


\section{A topological characterization}

Notation. Let $1 \leq \xi<\omega_{1}^{\mathrm{CK}}$. Theorem 4.1 in [L6] shows that if $A_{0}, A_{1}$ are disjoint $\Sigma_{1}^{1}$ relations on $\omega^{\omega}$, then $A_{0}$ is separable from $A_{1}$ by a $\operatorname{pot}\left(\boldsymbol{\Sigma}_{\xi}^{0}\right)$ set exactly when $A_{0} \cap{\overline{A_{1}}}^{\tau_{\xi}}=\emptyset$. We now define the versions of $A_{0} \cap{\overline{A_{1}}}^{\tau_{\xi}}$ for the classes $D_{\eta}\left(\boldsymbol{\Sigma}_{\xi}^{0}\right)$. So let $\varepsilon \in 2$ and $\eta<\omega_{1}^{\mathrm{CK}}$. We define $\bigcap_{\theta<0} F_{\theta, \xi}^{\varepsilon}:=\left(\omega^{\omega}\right)^{2}$, and, inductively,

$$
F_{\eta, \xi}^{\varepsilon}:={\overline{A_{|\operatorname{parity}(\eta)-\varepsilon|} \cap \bigcap_{\theta<\eta} F_{\theta, \xi}^{\varepsilon}}}_{\tau_{\xi}} .
$$

We will sometimes denote by $F_{\eta, \xi}^{\varepsilon}\left(A_{0}, A_{1}\right)$ the sets $F_{\eta, \xi}^{\varepsilon}$ previously defined. By induction, we can check that $F_{\eta, \xi}^{\varepsilon}\left(A_{1}, A_{0}\right)=F_{\eta, \xi}^{1-\varepsilon}\left(A_{0}, A_{1}\right)$.

Fix a bijection $l \mapsto\left((l)_{0},(l)_{1}\right)$ from $\omega$ onto $\omega^{2}$, with inverse map $(m, p) \mapsto<m, p>$. We define, for $u \in \omega^{\leq \omega}$ and $n \in \omega,(u)_{n} \in \omega^{\leq \omega}$ by $(u)_{n}(p):=u(<n, p>)$ if $<n, p><|u|$.

Theorem 3.2 Let $1 \leq \xi<\omega_{1}^{C K}, \eta=\lambda+2 k+\varepsilon<\omega_{1}^{C K}$ with $\lambda$ limit, $k \in \omega$ and $\varepsilon \in 2$, and $A_{0}, A_{1}$ be disjoint $\Sigma_{1}^{1}$ relations on $\omega^{\omega}$. Then the following are equivalent:

(1) the set $A_{0}$ is not separable from $A_{1}$ by a pot $\left(D_{\eta}\left(\Sigma_{\xi}^{0}\right)\right)$ set,

(2) the $\Sigma_{1}^{1}$ set $F_{\eta, \xi}^{\varepsilon}$ is not empty.

Proof. This result is essentially proved in [L8]. However, the formula for $F_{\eta, \xi}^{\varepsilon}$ is more concrete here, since the more general and abstract case of Wadge classes is considered in [L8]. So we give some details.

- In [Lo-SR], the following class of sets is introduced. Let $1 \leq \xi<\omega_{1}$ and $\boldsymbol{\Gamma}, \boldsymbol{\Gamma}^{\prime}$ be two classes of sets. Then $A \in S_{\xi}\left(\boldsymbol{\Gamma}, \boldsymbol{\Gamma}^{\prime}\right) \Leftrightarrow A=\bigcup_{p \geq 1}\left(A_{p} \cap C_{p}\right) \cup\left(B \backslash \bigcup_{p \geq 1} C_{p}\right)$, where $A_{p} \in \boldsymbol{\Gamma}, B \in \boldsymbol{\Gamma}^{\prime}$, and $\left(C_{p}\right)_{p \geq 1}$ is a sequence of pairwise disjoint $\Sigma_{\xi}^{0}$ sets. The authors prove the following:

$$
\begin{gathered}
\left.\boldsymbol{\Sigma}_{\xi}^{0}=S_{\xi}(\check{\{} \emptyset\},\{\emptyset\}\right), \\
D_{\theta+1}\left(\boldsymbol{\Sigma}_{\xi}^{0}\right)=S_{\xi}\left(\check{D}_{\theta}\left(\boldsymbol{\Sigma}_{\xi}^{0}\right), \boldsymbol{\Sigma}_{\xi}^{0}\right) \text { if } \theta<\omega_{1}, \\
D_{\lambda}\left(\boldsymbol{\Sigma}_{\xi}^{0}\right)=S_{\xi}\left(\bigcup_{p \geq 1} D_{\theta_{p}}\left(\boldsymbol{\Sigma}_{\xi}^{0}\right),\{\emptyset\}\right) \text { if } \lambda=\sup _{p \geq 1} \theta_{p} \text { is limit. }
\end{gathered}
$$

They also code the non self-dual Wadge classes of Borel sets by elements of $\omega_{1}^{\omega}$ as follows (we sometimes identify $\omega_{1}^{\omega}$ with $\left.\left(\omega_{1}^{\omega}\right)^{\omega}\right)$. The relations " $u$ is a second type description" and " $u$ describes $\Gamma$ " (written $u \in \mathcal{D}$ and $\boldsymbol{\Gamma}_{u}=\boldsymbol{\Gamma}$ - ambiguously) are the least relations satisfying the following properties.

(a) If $u=0^{\infty}$, then $u \in \mathcal{D}$ and $\boldsymbol{\Gamma}_{u}=\{\emptyset\}$.

(b) If $u=\xi \frown 1 \frown v$, with $v \in \mathcal{D}$ and $v(0)=\xi$, then $u \in \mathcal{D}$ and $\boldsymbol{\Gamma}_{u}=\check{\boldsymbol{\Gamma}}_{v}$.

(c) If $u=\xi \frown 2 \frown<u_{p}>$ satisfies $\xi \geq 1, u_{p} \in \mathcal{D}$, and $u_{p}(0) \geq \xi$ or $u_{p}(0)=0$, then $u \in \mathcal{D}$ and $\boldsymbol{\Gamma}_{u}=S_{\xi}\left(\bigcup_{p \geq 1} \boldsymbol{\Gamma}_{u_{p}}, \boldsymbol{\Gamma}_{u_{0}}\right)$.

They prove that $\boldsymbol{\Gamma}$ is a non self-dual Wadge class of Borel sets exactly when there is $u \in \mathcal{D}$ such that $\boldsymbol{\Gamma}\left(\omega^{\omega}\right)=\boldsymbol{\Gamma}_{u}\left(\omega^{\omega}\right)$. 
- In [L8], the elements of $\mathcal{D}$ are coded by elements of $\omega^{\omega}$. An inductive operator $\mathfrak{H}$ over $\omega^{\omega}$ is defined and there is a partial function $c: \omega^{\omega} \rightarrow \omega_{1}^{\omega}$ with $c\left[\mathfrak{H}^{\infty}\right]=\mathcal{D}$ (see Lemma 6.2 in [L8]). Another operator $\mathfrak{J}$ on $\left(\omega^{\omega}\right)^{3}$ is defined in [L8] to code the non self-dual Wadge classes of Borel sets and their elements (see Lemma 6.5 in [L8]). We will need a last inductive operator $\mathfrak{K}$, on $\left(\omega^{\omega}\right)^{6}$, to code the sets that will play the role of the $\Sigma_{1}^{1}$ sets $F_{\eta, \xi}^{\varepsilon}$ 's, via a universal set $\mathcal{U}$ for the class $\Pi_{1}^{1}\left(\omega^{\omega} \times \omega^{\omega}\right)$. More precisely, if $\left(\alpha, a_{0}, a_{1}, b_{0}, b_{1}, r\right) \in \mathfrak{K}^{\infty}$, then $b_{0}, b_{1}$ and $r$ are completely determined by $\left(\alpha, a_{0}, a_{1}\right)$ and in practice $\alpha$ will be in $\mathfrak{H}^{\infty}$, so that we will write $r=r\left(\alpha, a_{0}, a_{1}\right)=r\left(u, a_{0}, a_{1}\right)$ if $u=c(\alpha)$. Our $\Sigma_{1}^{1}$ sets $A_{0}, A_{1}$ are coded by $a_{0}, a_{1}$, in the sense that $A_{\varepsilon}=\neg \mathcal{U}_{a_{\varepsilon}}$. By Lemma 6.6 in [L8], there is a recursive map $\mathcal{A}:\left(\omega^{\omega}\right)^{2} \rightarrow \omega^{\omega}$ such that $\neg \mathcal{U}_{\mathcal{A}(\alpha, r)}=\left(\neg \mathcal{U}_{(r)_{0}}\right) \cap \bigcap_{p \geq 1}{\overline{\neg \mathcal{U}_{(r)} p}}^{\tau}$ if $\alpha \in \Delta_{1}^{1}$ codes a wellordering, where $r \mapsto\left((r)_{p}\right)_{p \in \omega}$ is a bijection from $\omega^{\omega}$ onto $\left(\omega^{\omega}\right)^{\omega}$. In the sequel, all the closures will be for $\tau_{\xi}$.

- We argue by induction on $\eta$. As $D_{0}\left(\boldsymbol{\Sigma}_{\xi}^{0}\right)=\{\emptyset\}, A_{0}$ is separable from $A_{1}$ by a $D_{0}\left(\boldsymbol{\Sigma}_{\xi}^{0}\right)$ set when $A_{0}=\emptyset$, which is equivalent to $F_{0, \xi}^{0}=\overline{A_{0}}=\emptyset$. As $D_{1}\left(\boldsymbol{\Sigma}_{\xi}^{0}\right)=\boldsymbol{\Sigma}_{\xi}^{0}, A_{0}$ is separable from $A_{1}$ by a $D_{1}\left(\boldsymbol{\Sigma}_{\xi}^{0}\right)$ set when $A_{0} \cap \overline{A_{1}}=\emptyset$ by Theorem 4.1 in [L6], which is equivalent to $F_{1, \xi}^{1}=\overline{A_{0} \cap \overline{A_{1}}}=\emptyset$.

Let us do these two basic cases in the spirit of the material from [L8] previously described, which will be done also for the other more complex cases.

- Note that $D_{0}\left(\boldsymbol{\Sigma}_{\xi}^{0}\right)=\{\emptyset\}=\boldsymbol{\Gamma}_{0 \infty}$. Let $\alpha \in \Delta_{1}^{1}$ such that $(\alpha)_{n}$ codes a wellordering of order type 0 for each $n \in \omega$. A look at the definition of $\mathfrak{H}$ shows that $\alpha \in \mathfrak{H}^{\infty}$. Another look at Definition 6.3 in [L8] shows that $\alpha$ is normalized (this will never be a problem in the sequel as well). Lemma 6.5 in [L8] gives $\beta, \gamma \in \omega^{\omega}$ with $(\alpha, \beta, \gamma) \in \mathfrak{J}^{\infty}$. Lemma 6.7 in [L8] gives $b_{0}, b_{1}, r \in \omega^{\omega}$ with $\left(\alpha, a_{1}, a_{0}, b_{0}, b_{1}, r\right) \in \mathfrak{K}^{\infty}$. By Theorem 6.10 in [L8], $A_{1}$ is separable from $A_{0}$ by a $\operatorname{pot}\left(\check{D}_{0}\left(\boldsymbol{\Sigma}_{\xi}^{0}\right)\right)$ set if and only if $\neg \mathcal{U}_{r}=\emptyset$. A look at the definition of $\mathfrak{K}$ shows that $r=a_{0}$, so that $\neg \mathcal{U}_{r}=A_{0}$.

- Now $\left.D_{1}\left(\boldsymbol{\Sigma}_{\xi}^{0}\right)=\boldsymbol{\Sigma}_{\xi}^{0}=S_{\xi}(\check{\{} \emptyset\},\{\emptyset\}\right)=S_{\xi}\left(\boldsymbol{\Gamma}_{010^{\infty}}, \boldsymbol{\Gamma}_{0^{\infty}}\right)=S_{\xi}\left(\bigcup_{p \geq 1} \boldsymbol{\Gamma}_{010^{\infty}}, \boldsymbol{\Gamma}_{0^{\infty}}\right)=\boldsymbol{\Gamma}_{v_{1}}$, where $v_{1}:=\xi 2<0^{\infty}, 010^{\infty}, 010^{\infty}, \ldots>$. As above, $A_{1}$ is separable from $A_{0}$ by a $\operatorname{pot}\left(\check{D}_{1}\left(\boldsymbol{\Sigma}_{\xi}^{0}\right)\right)$ set if and only if $\neg \mathcal{U}_{r}=\emptyset$. A look at the definition of $\mathfrak{K}$ shows that $r=b_{0}=\mathcal{A}\left(\alpha_{1},<a_{0}, a_{1}, a_{1}, \ldots>\right)$, where $\left|\alpha_{1}\right|=\xi$. Thus $\neg \mathcal{U}_{r}=A_{0} \cap \overline{A_{1}}$.

In the general case, there is $v_{\eta} \in \mathcal{D}$ such that $D_{\eta}\left(\Sigma_{\xi}^{0}\right)=\boldsymbol{\Gamma}_{v_{\eta}}$ and $A_{1}$ is separable from $A_{0}$ by a $\operatorname{pot}\left(\check{D}_{\eta}\left(\boldsymbol{\Sigma}_{\xi}^{0}\right)\right)$ set if and only if $\neg \mathcal{U}_{r\left(v_{\eta}, a_{1}, a_{0}\right)}=\emptyset$. Moreover,

(a) if $v_{\eta}=0^{\infty}$, then $r\left(v_{\eta}, a_{1}, a_{0}\right)=a_{0}$,

(b) if $v_{\eta}=\xi \frown 1 \frown v$, then $r\left(v_{\eta}, a_{1}, a_{0}\right)=a_{1}$,

(c) if $v_{\eta}=\xi \frown 2 \frown<u_{p}>$ and $r_{p}=r\left(u_{p}, a_{1}, a_{0}\right)$, then $r\left(v_{\eta}, a_{1}, a_{0}\right)=r\left(u_{0}, b_{1}, b_{0}\right)$, where by definition $b_{i}:=\mathcal{A}\left(\alpha_{1},<a_{i}, r_{1}, r_{2}, \ldots>\right)$.

It is enough to prove that $F_{\eta, \xi}^{\varepsilon}=\overline{\neg \mathcal{U}_{r\left(v_{\eta}, a_{1}, a_{0}\right)}}$, and we may assume that $\eta \geq 2$ by the previous discussion.

- If $\eta$ is a limit ordinal, then fix a sequence $\left(\eta_{p}\right)_{p \in \omega}$ of even ordinals cofinal in $\eta$. Note that

$$
D_{\eta}\left(\boldsymbol{\Sigma}_{\xi}^{0}\right)=S_{\xi}\left(\bigcup_{p \geq 1} D_{\eta_{p}}\left(\boldsymbol{\Sigma}_{\xi}^{0}\right),\{\emptyset\}\right)=S_{\xi}\left(\bigcup_{p \geq 1} \boldsymbol{\Gamma}_{u_{p}}, \boldsymbol{\Gamma}_{u_{0}}\right)=\boldsymbol{\Gamma}_{v_{\eta}},
$$

where $v_{\eta}=\xi \frown 2 \frown<u_{p}>$. 
Therefore, if $r_{p}:=r\left(u_{p}, a_{1}, a_{0}\right)$, then $F_{\theta_{p}, \xi}^{\varepsilon}=\overline{\neg \mathcal{U}_{r_{p}}}$ if $p \geq 1$, by the induction hypothesis. On the other hand, $r\left(u_{0}, b_{1}, b_{0}\right)=b_{0}$. But $b_{0}=\mathcal{A}\left(\alpha_{1},<a_{0}, r_{1}, r_{2}, \ldots>\right)$, so that

$$
\neg \mathcal{U}_{b_{0}}=\left(\neg \mathcal{U}_{a_{0}}\right) \cap \bigcap_{p \geq 1} \overline{\neg \mathcal{U}_{r_{p}}}
$$

as required.

- If $\eta=\theta+1$, then

$$
D_{\eta}\left(\boldsymbol{\Sigma}_{\xi}^{0}\right)=S_{\xi}\left(\check{D}_{\theta}\left(\boldsymbol{\Sigma}_{\xi}^{0}\right), \boldsymbol{\Sigma}_{\xi}^{0}\right)=S_{\xi}\left(\bigcup_{p \geq 1} \boldsymbol{\Gamma}_{u_{p}}, \boldsymbol{\Gamma}_{u_{0}}\right)=\boldsymbol{\Gamma}_{v_{\eta}},
$$

where $v_{\eta}=\xi^{\frown} 2 \frown\left\langle u_{p}>\right.$. Therefore, if $r_{p}:=r\left(u_{p}, a_{1}, a_{0}\right)$, then $F_{\theta, \xi}^{\varepsilon}=\overline{\neg \mathcal{U}_{r_{p}}}$ if $p \geq 1$, by the induction hypothesis (there is a double inversion of the superscript, one because the parity of $\theta$ is different from that of $\eta$, and the other one because there is a complement, so that the roles of $A_{0}, A_{1}$ are exchanged). By the case $\eta=1$ applied to $b_{0}$ and $b_{1}, \neg \mathcal{U}_{r\left(u_{0}, b_{1}, b_{0}\right)}=\neg \mathcal{U}_{b_{0}} \cap \overline{\neg \mathcal{U}_{b_{1}}}$. Note that

$$
\neg \mathcal{U}_{b_{i}}=\left(\neg \mathcal{U}_{a_{i}}\right) \cap \bigcap_{p \geq 1} \overline{\neg \mathcal{U}_{r_{p}}}=\left(\neg \mathcal{U}_{a_{i}}\right) \cap F_{\theta, \xi}^{\varepsilon}
$$

since $b_{i}=\mathcal{A}\left(\alpha_{1},<a_{i}, r_{1}, r_{2}, \ldots>\right)$. If $r:=r\left(v_{\eta}, a_{1}, a_{0}\right)$, then

$$
\neg U_{r}=\left(\neg \mathcal{U}_{a_{0}}\right) \cap F_{\theta, \xi}^{\varepsilon} \cap \overline{\neg \mathcal{U}_{a_{1}} \cap F_{\theta, \xi}^{\varepsilon}}=A_{0} \cap F_{\theta, \xi}^{\varepsilon},
$$

because $F_{\theta, \xi}^{\varepsilon}=\overline{A_{1} \cap \bigcap_{\rho<\theta} F_{\rho, \xi}^{\varepsilon}} \subseteq \overline{A_{1} \cap \overline{A_{1} \cap \bigcap_{\rho<\theta} F_{\rho, \xi}^{\varepsilon}}} \subseteq \overline{A_{1} \cap F_{\theta, \xi}^{\varepsilon}}$ (since the parity of $\theta$ is different from $\varepsilon$ ). Finally, $\overline{\neg U_{r}}=\overline{A_{0} \cap F_{\theta, \xi}^{\varepsilon}}=F_{\eta, \xi}^{\varepsilon}$, as required.

\section{The main result}

We set, for $\eta<\omega_{1}$ and $\varepsilon \in 2, \mathbb{B}_{\varepsilon}^{\eta}:=\left\{(0 \alpha, 1 \beta) \mid(\alpha, \beta) \in \mathbb{N}_{\varepsilon}^{\eta}\right\}$.

Theorem 3.3 Let $\eta \geq 1$ be a countable ordinal, $X$ be a Polish space, and $A_{0}, A_{1}$ be disjoint analytic relations on $X$ such that $A_{0} \cup A_{1}$ is quasi-acyclic. The following are equivalent:

(1) the set $A_{0}$ is not separable from $A_{1}$ by a pot $\left(D_{\eta}\left(\Sigma_{1}^{0}\right)\right)$ set,

(2) there is $\left(\mathbb{A}_{0}, \mathbb{A}_{1}\right) \in\left\{\left(\mathbb{N}_{0}^{\eta}, \mathbb{N}_{1}^{\eta}\right),\left(\mathbb{B}_{0}^{\eta}, \mathbb{B}_{1}^{\eta}\right)\right\}$ such that $\left(2^{\omega}, 2^{\omega}, \mathbb{A}_{0}, \mathbb{A}_{1}\right) \sqsubseteq\left(X, X, A_{0}, A_{1}\right)$, via a square map,

(3) $\left(2^{\omega}, 2^{\omega}, \mathbb{N}_{0}^{\eta}, \mathbb{N}_{1}^{\eta}\right) \sqsubseteq\left(X, X, A_{0}, A_{1}\right)$.

Proof. (1) $\Rightarrow(2)$ Let $\varepsilon:=\operatorname{parity}(\eta)$, and $\left(C_{p}\right)_{p \in \omega}$ be a witness for the quasi-acyclicity of $A_{0} \cup A_{1}$. We may assume that $X=\omega^{\omega}$. Indeed, we may assume that $X$ is zero-dimensional, and thus a closed subset of $\omega^{\omega}$. As $A_{0}$ is not separable from $A_{1}$ by a $\operatorname{pot}\left(D_{\eta}\left(\Sigma_{1}^{0}\right)\right)$ set in $X^{2}$, it is also the case in $\left(\omega^{\omega}\right)^{2}$, which gives $f: 2^{\omega} \rightarrow \omega^{\omega}$. As $\Delta\left(2^{\omega}\right) \subseteq \mathbb{N}_{0}^{\eta}$ and $\left\{(0 \alpha, 1 \alpha) \mid \alpha \in 2^{\omega}\right\} \subseteq \mathbb{B}_{0}^{\eta}$, the range of $\Delta\left(2^{\omega}\right)$ by $f \times f$ is a subset of $X^{2}$, so that $f$ takes values in $X$. We may also assume that $A_{0}, A_{1}$ are $\Sigma_{1}^{1}$, and that the relation " $(x, y) \in C_{p}$ " is $\Delta_{1}^{1}$ in $(x, y, p)$. By Theorem 3.2

$$
F_{\eta}^{\varepsilon}={\overline{A_{0} \cap \bigcap_{\theta<\eta} F_{\theta}^{\varepsilon}}}^{\tau_{1}}
$$

is a nonempty $\Sigma_{1}^{1}$ relation on $X$ (where $F_{\eta}^{\varepsilon}:=F_{\eta, 1}^{\varepsilon}$, for simplicity). 
We set, for $\theta \leq \eta, F_{\theta}:=A_{\mid \text {parity }(\theta)-\varepsilon \mid} \cap \bigcap_{\theta^{\prime}<\theta} F_{\theta^{\prime}}^{\varepsilon}$, so that $F_{\theta}^{\varepsilon}={\overline{F_{\theta}}}^{\tau_{1}}$. We put, for $\theta \leq \eta$,

$$
D_{\theta}:=\left\{\left(t_{s}^{0} w, t_{s}^{1} w\right) \in \mathcal{T}^{\eta} \mid \varphi(s)=\theta\right\}
$$

so that $\left(D_{\theta}\right)_{\theta \leq \eta}$ is a partition of $\mathcal{T}^{\eta}$. As $D_{\eta}=\Delta\left(2^{<\omega}\right), G_{l+1}:=s\left(\left(\bigcup_{\theta<\eta} D_{\theta}\right) \cap\left(2^{l+1} \times 2^{l+1}\right)\right)$ is a connected acyclic graph on $2^{l+1}$ for each $l \in \omega$.

Case $1 F_{\eta} \not \Delta(X)$.

Let $(x, y) \in F_{\eta} \backslash \Delta(X)$, and $O_{0}, O_{1}$ be disjoint $\Delta_{1}^{0}$ sets with $(x, y) \in O_{0} \times O_{1}$. We can replace $F_{\eta}, A_{0}$ and $A_{1}$ with their intersection with $O_{0} \times O_{1}$ if necessary and assume that they are contained in $O_{0} \times O_{1}$.

- We construct the following objects:

- sequences $\left(x_{s}\right)_{s \in 2^{<\omega}},\left(y_{s}\right)_{s \in 2<\omega}$ of points of $X$,

- sequences $\left(X_{s}\right)_{s \in 2^{<\omega}},\left(Y_{s}\right)_{s \in 2^{<\omega}}$ of $\Sigma_{1}^{1}$ subsets of $X$,

- a sequence $\left(U_{s, t}\right)_{(s, t) \in \mathcal{T}^{\eta}}$ of $\Sigma_{1}^{1}$ subsets of $X^{2}$, and $\Phi: \mathcal{T}^{\eta} \rightarrow \omega$.

We want these objects to satisfy the following conditions:

(1) $x_{s} \in X_{s} \wedge y_{s} \in Y_{s} \wedge\left(x_{s}, y_{t}\right) \in U_{s, t}$

(2) $X_{s \varepsilon} \subseteq X_{s} \subseteq \Omega_{X} \cap O_{0} \wedge Y_{s \varepsilon} \subseteq Y_{s} \subseteq \Omega_{X} \cap O_{1} \wedge U_{s, t} \subseteq C_{\Phi(s, t)} \cap \Omega_{X^{2}} \cap\left(X_{s} \times Y_{t}\right)$

(3) $\operatorname{diam}_{\mathrm{GH}}\left(X_{s}\right), \operatorname{diam}_{\mathrm{GH}}\left(Y_{s}\right), \operatorname{diam}_{\mathrm{GH}}\left(U_{s, t}\right) \leq 2^{-|s|}$

(4) $X_{s 0} \cap X_{s 1}=Y_{s 0} \cap Y_{s 1}=\emptyset$

(5) $U_{s \varepsilon, t \varepsilon} \subseteq U_{s, t}$

(6) $U_{s, t} \subseteq F_{\theta}$ if $(s, t) \in D_{\theta}$

- Assume that this has been done. Let $\alpha \in 2^{\omega}$. The sequence $\left(X_{\alpha \mid n}\right)_{n \in \omega}$ is a decreasing sequence of nonempty clopen subsets of $\Omega_{X}$ with vanishing diameters, which defines $f_{0}(\alpha) \in \bigcap_{n \in \omega} X_{\alpha \mid n}$. As the Gandy-Harrington topology is finer than the original topology, $f_{0}: 2^{\omega} \rightarrow O_{0}$ is continuous. By (4), $f_{0}$ is injective. Similarly, we define $f_{1}: 2^{\omega} \rightarrow O_{1}$ injective continuous. Finally, we define $f: 2^{\omega} \rightarrow X$ by $f(\varepsilon \alpha):=f_{\varepsilon}(\alpha)$, so that $f$ is also injective continuous since $O_{0}, O_{1}$ are disjoint.

If $(0 \alpha, 1 \beta) \in \mathbb{B}_{0}^{\eta}$, then there is $\theta \leq \eta$ of the same parity as $\eta$ such that $(\alpha, \beta) \mid n \in D_{\theta}$ if $n \geq n_{0}$. In this case, by (1)-(3) and (5)-(6), $\left(U_{(\alpha, \beta) \mid n}\right)_{n \geq n_{0}}$ is a decreasing sequence of nonempty clopen subsets of $A_{0} \cap \Omega_{X^{2}}$ with vanishing diameters, so that its intersection is a singleton $\{F(\alpha, \beta)\} \subseteq A_{0}$. As $\left(x_{\alpha \mid n}, y_{\beta \mid n}\right)$ converges (for $\Sigma_{X^{2}}$, and thus for $\left.\Sigma_{X}^{2}\right)$ to $F(\alpha, \beta),(f(0 \alpha), f(1 \beta))=F(\alpha, \beta) \in A_{0}$. If $(0 \alpha, 1 \beta) \in \mathbb{B}_{1}^{\eta}$, then the parity of $\theta$ is opposite to that of $\eta$ and, similarly, $(f(0 \alpha), f(1 \beta)) \in A_{1}$.

- So let us prove that the construction is possible. Note that $\left(t_{\emptyset}^{0}, t_{\emptyset}^{1}\right)=(\emptyset, \emptyset), \mathcal{T}^{\eta} \cap\left(2^{0} \times 2^{0}\right)=\{(\emptyset, \emptyset)\}$ and $(\emptyset, \emptyset) \in D_{\eta}$. Let $\left(x_{\emptyset}, y_{\emptyset}\right) \in F_{\eta} \cap \Omega_{X^{2}}$, and $\Phi(\emptyset, \emptyset) \in \omega$ such that $\left(x_{\emptyset}, y_{\emptyset}\right) \in C_{\Phi(\emptyset, \emptyset)}$. As $\Omega_{X^{2}} \subseteq \Omega_{X}^{2}$, $x_{\emptyset}, y_{\emptyset} \in \Omega_{X}$. We choose $\Sigma_{1}^{1}$ subsets $X_{\emptyset}, Y_{\emptyset}$ of $X$ with GH-diameter at most 1 such that

$$
\left(x_{\emptyset}, y_{\emptyset}\right) \in X_{\emptyset} \times Y_{\emptyset} \subseteq\left(\Omega_{X} \cap O_{0}\right) \times\left(\Omega_{X} \cap O_{1}\right),
$$

as well as a $\Sigma_{1}^{1}$ subset $U_{\emptyset, \emptyset}$ of $X^{2}$ with GH-diameter at most 1 such that

$$
\left(x_{\emptyset}, y_{\emptyset}\right) \in U_{\emptyset, \emptyset} \subseteq F_{\eta} \cap C_{\Phi(\emptyset, \emptyset)} \cap \Omega_{X^{2}} \cap\left(X_{\emptyset} \times Y_{\emptyset}\right),
$$

which completes the construction for the length $l=0$. 
Assume that we have constructed our objects for the sequences of length $l$. Let $u \in \omega^{<\omega}$ and $q \in \omega$ with $l+1=<u q>_{\eta}$, which gives $w \in \omega^{<\omega}$ with $\left(t_{u q}^{0}, t_{u q}^{1}\right)=\left(t_{u}^{0} w 0, t_{u}^{1} w 1\right)$. We set

$$
\begin{aligned}
& U:=\left\{x \in X \mid \exists\left(x_{s}^{\prime}\right)_{s \in 2^{l}} \in \Pi_{s \in 2^{l}} X_{s} \exists\left(y_{s}^{\prime}\right)_{s \in 2^{l}} \in \Pi_{s \in 2^{l}} Y_{s} \quad x=x_{t_{u}^{0} w}^{\prime} \wedge\right. \\
& \left.\forall(s, t) \in \mathcal{T}^{\eta} \cap\left(2^{l} \times 2^{l}\right)\left(x_{s}^{\prime}, y_{t}^{\prime}\right) \in U_{s, t}\right\}, \\
& V:=\left\{y \in X \mid \exists\left(x_{s}^{\prime}\right)_{s \in 2^{l}} \in \Pi_{s \in 2^{l}} X_{s} \exists\left(y_{s}^{\prime}\right)_{s \in 2^{l}} \in \Pi_{s \in 2^{l}} Y_{s} \quad y=y_{t_{u}^{1} w}^{\prime} \wedge\right. \\
& \left.\forall(s, t) \in \mathcal{T}^{\eta} \cap\left(2^{l} \times 2^{l}\right) \quad\left(x_{s}^{\prime}, y_{t}^{\prime}\right) \in U_{s, t}\right\} .
\end{aligned}
$$

Note that $U, V$ are $\Sigma_{1}^{1}$ and $\left(x_{t_{u}^{0} w}, y_{t_{u}^{1} w}\right) \in F_{\varphi(u)} \cap(U \times V) \subseteq \bigcap_{\theta<\varphi(u)}{\overline{F_{\theta}}}^{\tau_{1}} \cap(U \times V)$. This gives $\left(x_{t_{u}^{0} w 0}, y_{t_{u}^{1} w 1}\right) \in F_{\varphi(u q)} \cap(U \times V) \cap \Omega_{X^{2}}$. Let $\left(x_{s 0}\right)_{s \in 2^{l} \backslash\left\{t_{u}^{0} w\right\}}$ be witnesses for the fact that $x_{t_{u}^{0} w 0} \in U$, and $\left(x_{s 1}\right)_{s \in 2^{l} \backslash\left\{t_{u}^{1} w\right\}}$ be witnesses for the fact that $x_{t_{u}^{1} w 1} \in V$.

We need to show that $x_{s 0} \neq x_{s 1}$ (and similarly for $y_{s 0}$ and $y_{s 1}$ ). First observe that if $s \neq t \in 2^{l}$, then $x_{s \varepsilon} \in X_{s}$ and $x_{t \varepsilon^{\prime}} \in X_{t}$, so that $x_{s \varepsilon} \neq x_{t \varepsilon^{\prime}}$ by condition 4. Similarly, $y_{s \varepsilon} \neq y_{t \varepsilon^{\prime}}$. As $\varphi(u)$ and $\varphi(u q)$ do not have the same parity, there is $\epsilon \in 2$ such that $\left(x_{t_{u}^{0} w 0}, y_{t_{u}^{1} w 1}\right) \in A_{\epsilon}$ and

$$
\left(x_{t_{u}^{0} w 1}, y_{t_{u}^{1} w 1}\right) \in U_{t_{u}^{0} w, t_{u}^{1} w} \subseteq A_{1-\epsilon} .
$$

As $A_{0}$ and $A_{1}$ are disjoint, $x_{t_{u}^{0} w 0} \neq x_{t_{u}^{0} w 1}$. Similarly, $y_{t_{u}^{0} w 0} \neq y_{t_{u}^{0} w 1}$.

So we may assume that $l \geq 1$ and $s \neq t_{u}^{0} w$. The fact that $G_{l}$ is a connected graph provides a $G_{l}$-path from $s$ to $t_{u}^{0} w$. This path gives us two $s\left(A_{0} \cup A_{1}\right)$-paths by the definition of $U$ and $V$, one from $y_{s 0}$ to $x_{t_{u}^{0} w 0}$, and another one from $y_{s 1}$ to $x_{t_{u}^{0} w 1}$. Moreover, the same $\Phi\left(s^{\prime}, t^{\prime}\right)$ 's are involved in these two pathes since they are induced by the same $G_{l}$-path. Observe that $\left(x_{t_{u}^{0} w 0}, y_{t_{u}^{1} w 1}\right),\left(x_{t_{u}^{0} w 1}, y_{t_{u} w 1}\right)$ are in $s\left(A_{0} \cup A_{1}\right)$. Also, since $x_{s \varepsilon} \in O_{0}$ and $y_{t \varepsilon^{\prime}} \in O_{1}$, no " $x$ " is equal to no " $y$ ". Thus, by quasi-acyclicity, $y_{s 0} \neq y_{s 1}$. Similarly, one can prove that $x_{s 0} \neq x_{s 1}$. The following picture illustrates the situation when $l=1$ :

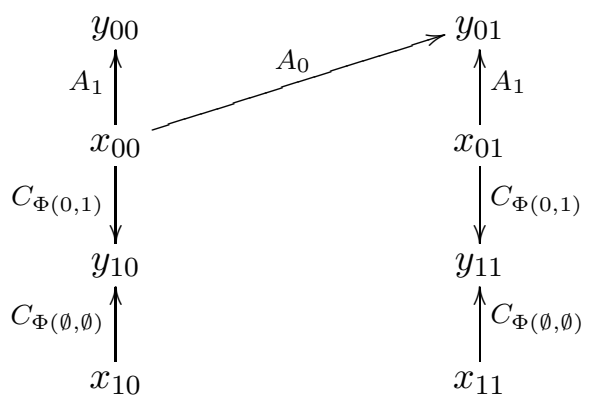

Let $\Phi\left(t_{u}^{0} w 0, t_{u}^{1} w 1\right) \in \omega$ such that $\left(x_{t_{u}^{0} w 0}, y_{t_{u}^{1} w 1}\right) \in C_{\Phi\left(t_{u}^{0} w 0, t_{u}^{1} w 1\right)}$, and $\Phi(s \varepsilon, t \varepsilon):=\Phi(s, t)$ if $(s, t)$ is in $\mathcal{T}^{\eta} \cap\left(2^{l} \times 2^{l}\right)$ and $\varepsilon \in 2$. It remains to take disjoint $\Sigma_{1}^{1}$ sets $X_{s 0}, X_{s 1} \subseteq X_{s}$ (respectively $Y_{s 0}, Y_{s 1} \subseteq Y_{s}$ ) with the required properties, as well as $V_{s \varepsilon, t \varepsilon^{\prime}}$, accordingly.

Case $2 F_{\eta} \subseteq \Delta(X)$.

Let us indicate the differences with Case 1. We set $S:=\left\{x \in X \mid(x, x) \in F_{\eta}\right\}$, which is a nonempty $\Sigma_{1}^{1}$ set by our assumption. 
- We construct the following objects:

- a sequence $\left(x_{s}\right)_{s \in 2<\omega}$ of points of $S$,

- a sequence $\left(X_{s}\right)_{s \in 2<\omega}$ of $\Sigma_{1}^{1}$ subsets of $X$,

- a sequence $\left(U_{s, t}\right)_{(s, t) \in \mathcal{T}^{\eta}}$ of $\Sigma_{1}^{1}$ subsets of $X^{2}$, and $\Phi: \mathcal{T}^{\eta} \rightarrow \omega$.

We want these objects to satisfy the following conditions:

(1) $x_{s} \in X_{s} \wedge\left(x_{s}, x_{t}\right) \in U_{s, t}$

(2) $X_{s \varepsilon} \subseteq X_{s} \subseteq \Omega_{X} \cap S \wedge U_{s, t} \subseteq C_{\Phi(s, t)} \cap \Omega_{X^{2}} \cap\left(X_{s} \times X_{t}\right)$

(3) $\operatorname{diam}_{\mathrm{GH}}\left(X_{s}\right), \operatorname{diam}_{\mathrm{GH}}\left(U_{s, t}\right) \leq 2^{-|s|}$

(4) $X_{s 0} \cap X_{s 1}=\emptyset$

(5) $U_{s \varepsilon, t \varepsilon} \subseteq U_{s, t}$

(6) $U_{s, t} \subseteq F_{\theta}$ if $(s, t) \in D_{\theta}$

- Assume that this has been done. As in Case 1, we get $f: 2^{\omega} \rightarrow X$ injective continuous such that $\mathbb{N}_{\epsilon}^{\eta} \subseteq(f \times f)^{-1}\left(A_{\epsilon}\right)$ for each $\epsilon \in 2$.

- So let us prove that the construction is possible. Let $\left(x_{\emptyset}, y_{\emptyset}\right) \in F_{\eta} \cap \Omega_{X^{2}}$. As $F_{\eta} \subseteq \Delta(X)$, $y_{\emptyset}=x_{\emptyset} \in S$. Let $\Phi(\emptyset, \emptyset) \in \omega$ with $\left(x_{\emptyset}, x_{\emptyset}\right) \in C_{\Phi(\emptyset, \emptyset)}$. As $\Omega_{X^{2}} \subseteq \Omega_{X}^{2}, x_{\emptyset} \in \Omega_{X}$. We choose a $\Sigma_{1}^{1}$ subset $X_{\emptyset}$ of $X$ with GH-diameter at most 1 such that $x_{\emptyset} \in X_{\emptyset} \subseteq \Omega_{X} \cap S$, as well as a $\Sigma_{1}^{1}$ subset $U_{\emptyset, \emptyset}$ of $X^{2}$ with GH-diameter at most 1 such that $\left(x_{\emptyset}, x_{\emptyset}\right) \in U_{\emptyset, \emptyset} \subseteq F_{\eta} \cap C_{\Phi(\emptyset, \emptyset)} \cap \Omega_{X^{2}} \cap\left(X_{\emptyset} \times X_{\emptyset}\right)$, which completes the construction for the length $l=0$.

For the inductive step, we set

$U:=\left\{x \in X \mid \exists\left(x_{s}^{\prime}\right)_{s \in 2^{l}} \in \Pi_{s \in 2^{l}} X_{s} \quad x=x_{t_{u}^{0} w}^{\prime} \wedge \forall(s, t) \in \mathcal{T}^{\eta} \cap\left(2^{l} \times 2^{l}\right)\left(x_{s}^{\prime}, x_{t}^{\prime}\right) \in U_{s, t}\right\}$, $V:=\left\{x \in X \mid \exists\left(x_{s}^{\prime}\right)_{s \in 2^{l}} \in \Pi_{s \in 2^{l}} X_{s} \quad x=x_{t_{u}^{1} w}^{\prime} \wedge \forall(s, t) \in \mathcal{T}^{\eta} \cap\left(2^{l} \times 2^{l}\right)\left(x_{s}^{\prime}, x_{t}^{\prime}\right) \in U_{s, t}\right\}$.

Again, we need to check that $x_{t_{q}^{0}} \neq x_{t_{q}^{1}}$ if $q \in \omega$. Note first that $A_{1} \cap S^{2}$ is irreflexive, since otherwise it contains $(x, x) \in A_{1} \cap F_{\eta} \subseteq A_{1} \cap A_{0}$. By construction, $\left(x_{t_{q}^{0}}, x_{t_{q}^{1}}\right) \in F_{\varphi(q)} \subseteq A_{1}$, and we are done.

$(2) \Rightarrow(3)$ Note that $\left(2^{\omega}, 2^{\omega}, \mathbb{N}_{0}^{\eta}, \mathbb{N}_{1}^{\eta}\right) \sqsubseteq\left(2^{\omega}, 2^{\omega}, \mathbb{B}_{0}^{\eta}, \mathbb{B}_{1}^{\eta}\right)$, with witnesses $\alpha \rightarrow 0 \alpha$ and $\beta \rightarrow 1 \beta$.

(3) $\Rightarrow$ (1) This comes from Lemma 3.1

Proposition 3.4 Let $\eta$ be a countable ordinal. The pairs $\left(\mathbb{N}_{0}^{\eta}, \mathbb{N}_{1}^{\eta}\right)$ and $\left(\mathbb{B}_{0}^{\eta}, \mathbb{B}_{1}^{\eta}\right)$ are incomparable for the square reduction.

Proof. There is no map $f: 2^{\omega} \rightarrow 2^{\omega}$ such that $\mathbb{N}_{\varepsilon}^{\eta} \subseteq(f \times f)^{-1}\left(\mathbb{B}_{\varepsilon}^{\eta}\right)$ since $\Delta\left(2^{\omega}\right)$ is a subset of $\mathbb{N}_{0}^{\eta}$.

There is no injection $f: 2^{\omega} \rightarrow 2^{\omega}$ for which there is $\alpha \in 2^{\omega}$ such that $f(0 \alpha)=f(1 \alpha)$. Using this fact, assume, towards a contradiction, that there is $f: 2^{\omega} \rightarrow 2^{\omega}$ injective continuous such that $\mathbb{B}_{\varepsilon}^{\eta} \subseteq(f \times f)^{-1}\left(\mathbb{N}_{\varepsilon}^{\eta}\right)$. Let $\left(0 t_{s}^{0} \gamma, 1 t_{s}^{1} \gamma\right) \in \mathbb{B}_{\varepsilon}^{\eta}$, so that $\left(f\left(0 t_{s}^{0} \gamma\right), f\left(1 t_{s}^{1} \gamma\right)\right)=\left(t_{v}^{0} \gamma^{\prime}, t_{v}^{1} \gamma^{\prime}\right) \in \mathbb{N}_{\varepsilon}^{\eta}$. 
We claim that $\varphi(s) \leq \varphi(v)$. We proceed by induction on $\varphi(s)$. Notice that is is obvious for $\varphi(s)=0$. Suppose that it holds for all $\theta<\varphi(s)$. Note that we can find $p_{k} \in \omega$ and $\gamma_{k} \in 2^{\omega}$ such that $\left(t_{s p_{k}}^{0} \gamma_{k}, t_{s p_{k}}^{1} \gamma_{k}\right) \in \mathbb{N}_{1-\varepsilon}^{\eta}$ and $\left(t_{s p_{k}}^{0} \gamma_{k}, t_{s p_{k}}^{1} \gamma_{k}\right) \rightarrow\left(t_{s}^{0} \gamma, t_{s}^{1} \gamma\right)$. By continuity,

$$
\left(t_{v_{k}}^{0} \gamma^{\prime}, t_{v_{k}}^{1} \gamma^{\prime}\right):=\left(f\left(0 t_{s p_{k}}^{0} \gamma_{k}\right), f\left(1 t_{s p_{k}}^{1} \gamma_{k}\right)\right) \rightarrow\left(t_{v}^{0} \gamma^{\prime}, t_{v}^{1} \gamma^{\prime}\right)
$$

In particular, for $k$ large, $\left(t_{v}^{0}, t_{v}^{1}\right) \subseteq\left(t_{v_{k}}^{0}, t_{v_{k}}^{1}\right)$. This implies that the sequence $v_{k}$ is a strict extension of $v$. Therefore $\varphi\left(v_{k}\right)<\varphi(v)$. By the induction hypothesis, $\varphi\left(s p_{k}\right) \leq \varphi\left(v_{k}\right)<\varphi(v)$. If $\varphi(s)=\theta+1$, then $\theta=\varphi\left(s p_{k}\right)<\varphi(v)$, so we are done. If $\varphi(s)$ is a limit ordinal, then $\left(\varphi\left(s p_{k}\right)\right)_{k \in \omega}$ is cofinal in it, so we are done too.

Finally, let $\alpha \in 2^{\omega}$, so that $(0 \alpha, 1 \alpha)=\left(0 t_{\emptyset}^{0} \alpha, 1 t_{\emptyset}^{1} \alpha\right) \in \mathbb{B}_{0}^{\eta}$. Then $(f(0 \alpha), f(1 \alpha))=\left(t_{v}^{0} \gamma^{\prime}, t_{v}^{1} \gamma^{\prime}\right)$ with $\varphi(v)=\eta$, so that $v=\emptyset$, which contradicts the injectivity of $f$.

\section{Consequences}

Lemma 3.5 Let $\boldsymbol{\Gamma}$ be a class of sets contained in $\boldsymbol{\Delta}_{2}^{0}$ which is either a Wadge class or $\boldsymbol{\Delta}_{2}^{0}$, X be a Polish space, and $A, B$ be disjoint analytic relations on $X$. Then exactly one of the following holds:

(a) the set $A$ is separable from $B$ by a pot $(\boldsymbol{\Gamma})$ set,

(b) there are $K_{\sigma}$ sets $A^{\prime} \subseteq A$ and $B^{\prime} \subseteq B$ such that $A^{\prime}$ is not separable from $B^{\prime}$ by a pot $(\boldsymbol{\Gamma})$ set.

Proof. Assume that (a) does not hold. Theorems 1.9 and 1.10 in [L8] give $\boldsymbol{\Sigma}_{2}^{0}$ relations $\mathbb{S}_{0}, \mathbb{S}_{1}$ on $2^{\omega}$ and $g, h: 2^{\omega} \rightarrow X$ continuous with $\mathbb{S}_{0} \subseteq(g \times h)^{-1}(A)$ and $\mathbb{S}_{1} \subseteq(g \times h)^{-1}(B)$. We set $A^{\prime}:=(g \times h)\left[\mathbb{S}_{0}\right]$ and $B^{\prime}:=(g \times h)\left[\mathbb{S}_{1}\right]$.

Corollary 3.6 Let $\eta<\omega_{1}, X$ be a Polish space, and $A, B$ be disjoint analytic relations on $X$ such that $A \cup B$ is s-acyclic or locally countable. Then exactly one of the following holds:

(a) the set $A$ is separable from $B$ by a pot $\left(D_{\eta}\left(\Sigma_{1}^{0}\right)\right)$ set,

(b) $\left(2^{\omega}, 2^{\omega}, \mathbb{N}_{0}^{\eta}, \mathbb{N}_{1}^{\eta}\right) \sqsubseteq(X, X, A, B)$ if $\eta \geq 1$ and $\left(1,1, \mathbb{N}_{0}^{\eta}, \mathbb{N}_{1}^{\eta}\right) \sqsubseteq(X, X, A, B)$ if $\eta=0$.

Proof. By Lemma 3.1, $\mathbb{N}_{0}^{\eta}$ is not separable from $\mathbb{N}_{1}^{\eta}$ by a $\operatorname{pot}\left(D_{\eta}\left(\boldsymbol{\Sigma}_{1}^{0}\right)\right)$ set. This shows that (a) and (b) cannot hold simultaneously. So assume that (a) does not hold. We may assume that $\eta \geq 1$. By Lemma 3.5. we may assume that $A, B$ are $\boldsymbol{\Sigma}_{2}^{0}$. By Lemma 2.2, we may also assume that $A \cup B$ is quasi-acyclic. It remains to apply Theorem 3.3 .

Corollary 3.7 Let $\eta$ be a countable ordinal, $X, Y$ be Polish spaces, and A, B be disjoint analytic subsets of $X \times Y$ such that $A \cup B$ is locally countable. Then exactly one of the following holds:

(a) the set $A$ is separable from $B$ by a pot $\left(D_{\eta}\left(\Sigma_{1}^{0}\right)\right)$ set,

(b) $\left(2^{\omega}, 2^{\omega}, \mathbb{N}_{0}^{\eta}, \mathbb{N}_{1}^{\eta}\right) \sqsubseteq(X, Y, A, B)$ if $\eta \geq 1$ and $\left(1,1, \mathbb{N}_{0}^{\eta}, \mathbb{N}_{1}^{\eta}\right) \sqsubseteq(X, Y, A, B)$ if $\eta=0$.

Proof. We may assume that $\eta \geq 1$. As in the proof of Corollary 3.6, (a) and (b) cannot hold simultaneously. So assume that (a) does not hold. We put $Z:=X \oplus Y, A^{\prime}:=\left\{((x, 0),(y, 1)) \in Z^{2} \mid(x, y) \in A\right\}$ and $B^{\prime}:=\left\{((x, 0),(y, 1)) \in Z^{2} \mid(x, y) \in B\right\}$. Then $Z$ is Polish, $A^{\prime}, B^{\prime}$ are disjoint analytic relations on $Z, A^{\prime} \cup B^{\prime}$ is locally countable, and $A^{\prime}$ is not separable from $B^{\prime}$ by $\operatorname{art}\left(D_{\eta}\left(\Sigma_{1}^{0}\right)\right)$ set. 
Corollary 3.6 gives $f^{\prime}, g^{\prime}: 2^{\omega} \rightarrow Z$ injective continuous such that $\mathbb{N}_{0}^{\eta} \subseteq\left(f^{\prime} \times g^{\prime}\right)^{-1}\left(A^{\prime}\right)$, and also $\mathbb{N}_{1}^{\eta} \subseteq\left(f^{\prime} \times g^{\prime}\right)^{-1}\left(B^{\prime}\right)$. We set $f(\alpha):=\Pi_{0}\left[f^{\prime}(\alpha)\right]$, and $g(\beta):=\Pi_{0}\left[g^{\prime}(\beta)\right]$. As $\Delta\left(2^{\omega}\right) \subseteq \mathbb{N}_{0}^{\eta}, f^{\prime}$ takes values in $X \times\{0\}$ and $g^{\prime}$ takes values in $Y \times\{1\}$. This implies that $f: 2^{\omega} \rightarrow X, g: 2^{\omega} \rightarrow Y$ are injective continuous. We are done since $\mathbb{N}_{0}^{\eta} \subseteq(f \times g)^{-1}(A)$ and $\mathbb{N}_{1}^{\eta} \subseteq(f \times g)^{-1}(B)$.

Notation. If $A$ is a relation on $2^{\omega}$, then we set $G_{A}:=\{(0 \alpha, 1 \beta) \mid(\alpha, \beta) \in A\}$.

Lemma 3.8 Let $A$ be an antisymmetric s-acyclic relation on $2^{\omega}$. Then $G_{A}$ is s-acyclic.

Proof. We argue by contradiction, which gives $n \geq 2$ and an injective $s\left(G_{A}\right)$-path $\left(\varepsilon_{i} z_{i}\right)_{i \leq n}$ such that $\left(\varepsilon_{0} z_{0}, \varepsilon_{n} z_{n}\right) \in s\left(G_{A}\right)$. This implies that $\varepsilon_{i} \neq \varepsilon_{i+1}$ if $i<n$ and $n$ is odd. Thus $\left(z_{i}\right)_{i \leq n}$ is a $s(A)$ path such that $\left(z_{2 j}\right)_{2 j \leq n}$ and $\left(z_{2 j+1}\right)_{2 j+1 \leq n}$ are injective and $\left(z_{0}, z_{n}\right) \in s(A)$. As $s(A)$ is acyclic, the sequence $\left(z_{i}\right)_{i \leq n}$ is not injective. We erase $z_{2 j+1}$ from this sequence if $z_{2 j+1} \in\left\{z_{2 j}, z_{2 j+2}\right\}$ and $2 j+1 \leq n$, which gives a sequence $\left(z_{i}^{\prime}\right)_{i \leq n^{\prime}}$ which is still a $s(A)$-path with $\left(z_{0}^{\prime}, z_{n^{\prime}}^{\prime}\right) \in s(A)$, and moreover satisfies $z_{i}^{\prime} \neq z_{i+1}^{\prime}$ if $i<n^{\prime}$.

If $n^{\prime}<2$, then $n=3, z_{0}=z_{1}$ and $z_{2}=z_{3}$. As $A$ is antisymmetric and $\varepsilon_{3}=\varepsilon_{1} \neq \varepsilon_{2}=\varepsilon_{0}$, we get $z_{0}=z_{2}$, which is absurd. If $n^{\prime} \geq 2$, then $\left(z_{i}^{\prime}\right)_{i \leq n^{\prime}}$ is not injective again. We choose a subsequence of it with at least three elements, made of consecutive elements, such that the first and the last elements are equal, and of minimal length with these properties. The acyclicity of $s(A)$ implies that this subsequence has exactly three elements, say $\left(z_{i}^{\prime}, z_{i+1}^{\prime}, z_{i+2}^{\prime}=z_{i}^{\prime}\right)$.

If $z_{i}^{\prime}=z_{2 j+1}$, then $z_{i+1}^{\prime}=z_{2 j+2}, z_{i+2}^{\prime}=z_{2 j+4}$ and $z_{2 j+3}=z_{2 j+2}$. As $A$ is antisymmetric and $\varepsilon_{2 j+3}=\varepsilon_{2 j+1} \neq \varepsilon_{2 j+2}=\varepsilon_{2 j+4}$, we get $z_{2 j+2}=z_{2 j+4}$, which is absurd. If $z_{i}^{\prime}=z_{2 j}$, then $z_{i+1}^{\prime}=z_{2 j+2}$, and $z_{i+2}^{\prime}=z_{2 j+3}$. As $A$ is antisymmetric and $\varepsilon_{2 j+3}=\varepsilon_{2 j+1} \neq \varepsilon_{2 j+2}=\varepsilon_{2 j}$, we get $z_{2 j}=z_{2 j+2}$, which is absurd.

Corollary 3.9 Let $\eta \geq 1$ be a countable ordinal, $X$ be a Polish space, and A, B be disjoint analytic relations on $X$. The following are equivalent:

(1) there is an s-acyclic relation $R \in \Sigma_{1}^{1}$ such that $A \cap R$ is not separable from $B \cap R$ by a pot $\left(D_{\eta}\left(\Sigma_{1}^{0}\right)\right)$ set,

(2) there is a locally countable relation $R \in \Sigma_{1}^{1}$ such that $A \cap R$ is not separable from $B \cap R$ by a $\operatorname{pot}\left(D_{\eta}\left(\Sigma_{1}^{0}\right)\right)$ set,

(3) $\left(2^{\omega}, 2^{\omega}, \mathbb{N}_{0}^{\eta}, \mathbb{N}_{1}^{\eta}\right) \sqsubseteq(X, X, A, B)$,

(4) there is $\left(\mathbb{A}_{0}, \mathbb{A}_{1}\right) \in\left\{\left(\mathbb{N}_{0}^{\eta}, \mathbb{N}_{1}^{\eta}\right),\left(\mathbb{B}_{0}^{\eta}, \mathbb{B}_{1}^{\eta}\right)\right\}$ such that $\left(2^{\omega}, 2^{\omega}, \mathbb{A}_{0}, \mathbb{A}_{1}\right) \sqsubseteq(X, X, A, B)$, via a square map.

A similar result holds for $\eta=0$ with 1 instead of $2^{\omega}$.

Proof. (1) $\Rightarrow(3),(4)$ and (2) $\Rightarrow(3),(4)$ This is a consequence of Corollary 3.6 and its proof.

(4) $\Rightarrow$ (1) By the remarks before Lemma 3.1, $\mathbb{N}_{0}^{\eta} \cup \mathbb{N}_{1}^{\eta}$ has s-acyclic levels. This implies that $\mathbb{N}_{0}^{\eta} \cup \mathbb{N}_{1}^{\eta}$ is s-acyclic. As $\mathbb{N}_{0}^{\eta} \cup \mathbb{N}_{1}^{\eta}$ is antisymmetric, $\mathbb{B}_{0}^{\eta} \cup \mathbb{B}_{1}^{\eta}$ is s-acyclic too, by Lemma3.8. Thus we can take $R:=(f \times f)\left[\mathbb{A}_{0} \cup \mathbb{A}_{1}\right]$ since the s-acyclicity is preserved by images by the square of an injection, and by Lemma 3.1 
(4) $\Rightarrow$ (2) We can take $R:=(f \times f)\left[\mathbb{A}_{0} \cup \mathbb{A}_{1}\right]$ since $\mathbb{A}_{0} \cup \mathbb{A}_{1}$ is locally countable, by Lemma 3.1.

(3) $\Rightarrow$ (2) We can take $R:=(f \times f)\left[\mathbb{N}_{0}^{\eta} \cup \mathbb{N}_{1}^{\eta}\right]$ since $\mathbb{N}_{0}^{\eta} \cup \mathbb{N}_{1}^{\eta}$ is locally countable, by Lemma 3.1

Remark. There is a version of Corollary 3.9 for $\check{D}_{\eta}\left(\Sigma_{1}^{0}\right)$ instead of $D_{\eta}\left(\Sigma_{1}^{0}\right)$, obtained by exchanging the roles of $A$ and $B$. This symmetry is also present in Theorem 3.3 .

We now give some complements when $\eta=1$. At the beginning of this section, we mentioned the fact that our examples are in the style of $\mathbb{G}_{0}$. If $\eta=1$, then $\mathbb{G}_{0}$ itself is involved.

Corollary 3.10 Let $X$ be a Polish space, and $A, B$ be disjoint analytic relations on $X$ such that

- either $A \cup B$ is s-acyclic or locally countable,

- or A is contained in a potentially closed s-acyclic or locally countable relation.

Then exactly one of the following holds:

(a) the set $A$ is separable from $B$ by a pot $\left(\Pi_{1}^{0}\right)$ set,

(b) $\left(2^{\omega}, 2^{\omega}, \mathbb{G}_{0}, \Delta\left(2^{\omega}\right)\right) \sqsubseteq(X, X, A, B)$.

Corollary 3.11 Let $X, Y$ be Polish spaces, and $A, B$ be disjoint analytic subsets of $X \times Y$ such that $A \cup B$ is locally countable or $A$ is contained in a potentially closed locally countable set. Then exactly one of the following holds:

(a) the set $A$ is separable from $B$ by a pot $\left(\Pi_{1}^{0}\right)$ set,

(b) $\left(2^{\omega}, 2^{\omega}, \mathbb{G}_{0}, \Delta\left(2^{\omega}\right)\right) \sqsubseteq(X, Y, A, B)$.

\section{The class $\Delta\left(D_{\eta}\left(\Sigma_{1}^{0}\right)\right)$}

\section{Examples}

Notation. We set, for each countable ordinal $\eta \geq 1$ and each $\varepsilon \in 2$,

$$
\mathbb{S}_{\varepsilon}^{\eta}:=\left\{\left(t_{s}^{0} \gamma, t_{s}^{1} \gamma\right) \mid s \in T_{\eta} \backslash\{\emptyset\} \wedge \text { parity }(|s|)=1-|\operatorname{parity}(s(0))-\varepsilon| \wedge \gamma \in 2^{\omega}\right\} .
$$

Lemma 4.1 Let $\eta \geq 1$ be a countable ordinal, and $C$ be a nonempty clopen subset of $2^{\omega}$. Then $\mathbb{S}_{0}^{\eta} \cap C^{2}$ is not separable from $\mathbb{S}_{1}^{\eta} \cap C^{2}$ by a pot $\left(\Delta\left(D_{\eta}\left(\Sigma_{1}^{0}\right)\right)\right)$ set.

Proof. We use the notation in the proof of Lemma 3.1. We argue by contradiction, which gives $P$ in $\operatorname{pot}\left(\Delta\left(D_{\eta}\left(\Sigma_{1}^{0}\right)\right)\right)$, and a dense $G_{\delta}$ subset of $2^{\omega}$ such that $P \cap G^{2}, G^{2} \backslash P \in D_{\eta}\left(\Sigma_{1}^{0}\right)\left(G^{2}\right)$. So let, for each $\varepsilon \in 2,\left(O_{\theta}^{\varepsilon}\right)_{\theta<\eta}$ be a sequence of open relations on $2^{\omega}$ such that

$$
P \cap G^{2}=\left(\underset{\theta<\eta, p a r i t y}{\bigcup_{(\theta) \neq \operatorname{parity}(\eta)}} O_{\theta}^{0} \backslash\left(\bigcup_{\theta^{\prime}<\theta} O_{\theta^{\prime}}^{0}\right)\right) \cap G^{2}
$$

and $G^{2} \backslash P=\left(\bigcup_{\theta<\eta, \text { parity }(\theta) \neq \operatorname{parity}(\eta)} O_{\theta}^{1} \backslash\left(\bigcup_{\theta^{\prime}<\theta} O_{\theta^{\prime}}^{1}\right)\right) \cap G^{2}$. 
- Note that $\mathbb{S}_{\varepsilon}^{\eta}=\bigcup_{s \in T_{\eta} \backslash\{\emptyset\} \text {,parity }(|s|)=1-|\operatorname{parity}(s(0))-\varepsilon|} \operatorname{Gr}\left(f_{s}\right)$. Let us show that if $\theta \leq \eta, s \in T_{\eta}$ and $\varphi(s)=\theta$, then $\operatorname{Gr}\left(f_{s}\right) \cap(C \cap G)^{2} \subseteq \neg O_{\theta}^{1-\text { parity }(s(0))}$ if $\theta<\eta$, and $\operatorname{Gr}\left(f_{s}\right) \cap(C \cap G)^{2}$ is disjoint from $\bigcup_{\theta^{\prime}<\theta}\left(O_{\theta^{\prime}}^{0} \cup O_{\theta^{\prime}}^{1}\right)$ if $\theta=\eta$. The objects $s=\emptyset$ and $\theta=\eta$ will give the contradiction.

- We argue by induction on $\theta$. Note that $\operatorname{Gr}\left(f_{s}\right) \cap(C \cap G)^{2} \subseteq \mathbb{S}_{1-\mid \text { parity }(|s|)-\operatorname{parity}(s(0)) \mid}^{\eta} \cap G^{2}$ if $\theta=0$ since $s \neq \emptyset$. As $\mathbb{S}_{\varepsilon}^{\eta} \cap G^{2} \subseteq \neg O_{0}^{\mid \text {parity }(\eta)-\varepsilon \mid}$ for each $\varepsilon \in 2$ and $|s|$ has the same parity as $\eta$ if $\theta=0$, we are done.

- Assume that the result has been proved for $\theta^{\prime}<\theta$. If $\theta$ is the successor of $\theta^{\prime}$, then the induction assumption implies that $\operatorname{Gr}\left(f_{s q}\right) \cap(C \cap G)^{2} \subseteq \neg O_{\theta^{\prime}}^{1-p a r i t y}((s q)(0))$ for each $q$. We set, for each $\varepsilon \in 2$, $C_{s}^{\varepsilon}:=\bigcup_{k \in \omega} \operatorname{Gr}\left(f_{s(2 k+\varepsilon)}\right)$, so that $\operatorname{Gr}\left(f_{s}\right) \subseteq \overline{C_{s}^{\varepsilon}}$, by the choice of $\psi$. If $s=\emptyset$, then

$$
C_{\emptyset}^{\varepsilon} \cap(C \cap G)^{2} \subseteq \neg O_{\theta^{\prime}}^{1-\varepsilon},
$$

$\operatorname{Gr}\left(f_{s}\right) \cap(C \cap G)^{2} \subseteq \overline{C_{\emptyset}^{\varepsilon}} \cap(C \cap G)^{2} \subseteq \overline{C_{\emptyset}^{\varepsilon} \cap(C \cap G)^{2}} \subseteq \neg O_{\theta^{\prime}}^{1-\varepsilon}$, which gives the desired inclusion for $\theta=\eta$.

$$
\begin{aligned}
& \text { If } s \neq \emptyset \text {, then } \operatorname{Gr}\left(f_{s q}\right) \cap(C \cap G)^{2} \subseteq \neg O_{\theta^{\prime}}^{1-\operatorname{parity}(s(0))} \text { for each } q \text {, so that } \\
& \qquad \operatorname{Gr}\left(f_{s}\right) \cap(C \cap G)^{2} \subseteq \overline{C_{s}} \cap(C \cap G)^{2} \subseteq \overline{C_{s} \cap(C \cap G)^{2}} \subseteq \neg O_{\theta^{\prime}}^{1-\operatorname{parity}(s(0))} .
\end{aligned}
$$

Thus

$\operatorname{Gr}\left(f_{s}\right) \cap(C \cap G)^{2} \subseteq\left(G^{2} \backslash O_{\theta^{\prime}}^{1-\operatorname{parity}(s(0))}\right) \cap \neg\left(O_{\theta}^{1-\operatorname{parity}(s(0))} \backslash O_{\theta^{\prime}}^{1-\operatorname{parity}(s(0))}\right) \subseteq \neg O_{\theta}^{1-\operatorname{parity}(s(0))}$ since $\operatorname{parity}(\theta)=|\operatorname{parity}(|s|)-\operatorname{parity}(\eta)|$.

- If $\theta$ is limit, then $(\varphi(s n))_{n \in \omega}$ is cofinal in $\varphi(s)$, and $\operatorname{Gr}\left(f_{s n}\right) \cap(C \cap G)^{2} \subseteq \neg O_{\varphi(s n)}^{1-\text { parity }((s n)(0))}$, by the induction assumption. If $\theta_{0}<\varphi(s)$, then there is $n\left(\theta_{0}\right)$ such that $\varphi(s n)>\theta_{0}$ if $n \geq n\left(\theta_{0}\right)$. Thus $\operatorname{Gr}\left(f_{s n}\right) \cap(C \cap G)^{2} \subseteq \neg O_{\theta_{0}}^{1-\operatorname{parity}((s n)(0))}$ if $n \geq n\left(\theta_{0}\right)$. If $s=\emptyset$, then, for each $\varepsilon \in 2$,

$$
\begin{aligned}
\operatorname{Gr}\left(f_{s}\right) \cap(C \cap G)^{2} & \subseteq(C \cap G)^{2} \cap \overline{C_{s}^{\varepsilon}} \backslash C_{s}^{\varepsilon}=\overline{C_{s}^{\varepsilon} \cap(C \cap G)^{2}} \backslash C_{s}^{\varepsilon} \\
& \subseteq \bigcup_{n \geq n\left(\theta_{0}\right), \operatorname{parity}(n)=\varepsilon} \operatorname{Gr}\left(f_{s n}\right) \cap(C \cap G)^{2} \subseteq \neg O_{\theta_{0}}^{1-\varepsilon} .
\end{aligned}
$$

Thus $\operatorname{Gr}\left(f_{s}\right) \cap(C \cap G)^{2} \subseteq \neg\left(\bigcup_{\theta^{\prime}<\eta}\left(O_{\theta^{\prime}}^{0} \cup O_{\theta^{\prime}}^{1}\right)\right)$. If $s \neq \emptyset$, then $\operatorname{Gr}\left(f_{s n}\right) \cap(C \cap G)^{2} \subseteq \neg O_{\theta_{0}}^{1-\text { parity }(s(0))}$ for each $n$, so that $\operatorname{Gr}\left(f_{s}\right) \cap(C \cap G)^{2} \subseteq \overline{C_{s}} \cap(C \cap G)^{2} \subseteq \overline{C_{s} \cap(C \cap G)^{2}} \subseteq \neg O_{\theta_{0}}^{1-\operatorname{parity}(s(0))}$. As $\operatorname{parity}(|s|)=\operatorname{parity}(\eta), \operatorname{Gr}\left(f_{s}\right) \cap(C \cap G)^{2} \subseteq \neg O_{\theta}^{1-\operatorname{parity}(s(0))}$ as above.

\section{$\underline{\text { A topological characterization }}$}

Notation. We define, for $1 \leq \xi<\omega_{1}^{\mathrm{CK}}$ and $\eta<\omega_{1}^{\mathrm{CK}}, \bigcap_{\theta<0} G_{\theta, \xi}:=\left(\omega^{\omega}\right)^{2}$, and, inductively,

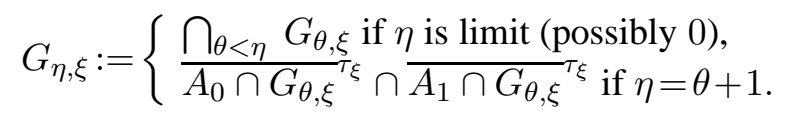


Theorem 4.2 Let $1 \leq \xi<\omega_{1}^{C K}, 1 \leq \eta=\lambda+2 k+\varepsilon<\omega_{1}^{C K}$ with $\lambda$ limit, $k \in \omega$ and $\varepsilon \in 2$, and $A_{0}, A_{1}$ be disjoint $\Sigma_{1}^{1}$ relations on $\omega^{\omega}$. Then the following are equivalent:

(1) the set $A_{0}$ is not separable from $A_{1}$ by a pot $\left(\Delta\left(D_{\eta}\left(\Sigma_{\xi}^{0}\right)\right)\right)$ set,

(2) the $\Sigma_{1}^{1}$ set $G_{\eta, \xi}$ is not empty.

Proof. The proof is in the spirit of that of Theorem 3.2. The proof of Theorem 1.10.(2) in [L8] gives $\alpha$ suitable such that $c(\alpha)$ codes the class $D_{\eta}\left(\boldsymbol{\Sigma}_{\xi}^{0}\right)$. By Theorem 6.26 in [L8] and Theorem 3.2, (1) is equivalent to $R^{\prime}\left(\alpha, a_{0}, a_{1}\right) \neq \emptyset$, where

$$
R^{\prime}\left(\alpha, a_{0}, a_{1}\right):=\left\{\begin{array}{l}
F_{\theta, \xi}^{0} \cap F_{\theta, \xi}^{1} \text { if } \eta=\theta+1, \\
\bigcap_{p \geq 1} F_{\theta_{p}, \xi}^{0} \text { if } \eta=\sup _{p \geq 1} \theta_{p} \text { is limit } \wedge \theta_{p} \text { is odd. }
\end{array}\right.
$$

So it is enough to prove that

$$
G_{\eta, \xi}=\left\{\begin{array}{l}
F_{\theta, \xi}^{0} \cap F_{\theta, \xi}^{1} \text { if } \eta=\theta+1, \\
\bigcap_{p \geq 1} F_{\theta_{p}, \xi}^{0} \text { if } \eta=\sup _{p \geq 1} \theta_{p} \text { is limit } \wedge \theta_{p} \text { is odd. }
\end{array}\right.
$$

We argue by induction on $\eta$. Note first that $G_{1, \xi}=\overline{A_{0}} \cap \overline{A_{1}}=F_{0, \xi}^{0} \cap F_{0, \xi}^{1}$. Then, inductively,

$$
\begin{aligned}
G_{\theta+2, \xi} & =\overline{A_{0} \cap G_{\theta+1, \xi}} \cap \overline{A_{1} \cap G_{\theta+1, \xi}}=\overline{A_{0} \cap F_{\theta, \xi}^{0} \cap F_{\theta, \xi}^{1}} \cap \overline{A_{1} \cap F_{\theta, \xi}^{0} \cap F_{\theta, \xi}^{1}} \\
& =\overline{A_{0} \cap F_{\theta, \xi}^{1-\text { parity }(\theta)}} \cap \overline{A_{1} \cap F_{\theta, \xi}^{\text {parity }(\theta)}}=F_{\theta+1, \xi}^{0} \cap F_{\theta+1, \xi}^{1} .
\end{aligned}
$$

If $\lambda$ is limit, then

$$
\begin{aligned}
& G_{\lambda+1, \xi}=\overline{A_{0} \cap G_{\lambda, \xi}} \cap \overline{A_{1} \cap G_{\lambda, \xi}}=\overline{A_{0} \cap \bigcap_{\theta<\lambda} G_{\theta, \xi}} \cap \overline{A_{1} \cap \bigcap_{\theta<\lambda} G_{\theta, \xi}} \\
& =\overline{A_{0} \cap \bigcap_{\theta<\lambda} G_{\theta+1, \xi}} \cap \overline{A_{1} \cap \bigcap_{\theta<\lambda} G_{\theta+1, \xi}}
\end{aligned}
$$

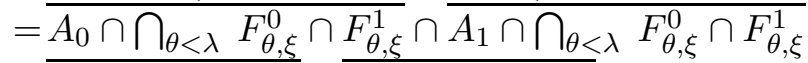

$$
\begin{aligned}
& =\overline{A_{0} \cap \bigcap_{\theta<\lambda} F_{\theta, \xi}^{0}} \cap \overline{A_{1} \cap \bigcap_{\theta<\lambda} F_{\theta, \xi}^{1}}=F_{\lambda, \xi}^{0} \cap F_{\lambda, \xi}^{1}
\end{aligned}
$$

and $G_{\lambda, \xi}=\bigcap_{\theta<\lambda} G_{\theta, \xi}=\bigcap_{\theta<\lambda} G_{\theta+1, \xi}=\bigcap_{\theta<\lambda} F_{\theta, \xi}^{0} \cap F_{\theta, \xi}^{1}=\bigcap_{\theta<\lambda} F_{\theta, \xi}^{0}=\bigcap_{p \geq 1} F_{\theta p}^{0}$.

\section{The main result}

We prove a version of Theorem 3.3 for the class $\Delta\left(D_{\eta}\left(\Sigma_{1}^{0}\right)\right)$. We set, for $1 \leq \eta<\omega_{1}$ and $\varepsilon \in 2$, $\mathbb{C}_{\varepsilon}^{\eta}:=\left\{(0 \alpha, 1 \beta) \mid(\alpha, \beta) \in \mathbb{S}_{\varepsilon}^{\eta}\right\}$.

Theorem 4.3 Let $\eta \geq 1$ be a countable ordinal, $X$ be a Polish space, and $A_{0}, A_{1}$ be disjoint analytic relations on $X$ such that $A_{0} \cup A_{1}$ is contained in a potentially closed quasi-acyclic relation. The following are equivalent:

(1) the set $A_{0}$ is not separable from $A_{1}$ by a pot $\left(\Delta\left(D_{\eta}\left(\Sigma_{1}^{0}\right)\right)\right)$ set,

(2) there is $\left(\mathbb{A}_{0}, \mathbb{A}_{1}\right) \in\left\{\left(\mathbb{N}_{1}^{\eta}, \mathbb{N}_{0}^{\eta}\right),\left(\mathbb{B}_{1}^{\eta}, \mathbb{B}_{0}^{\eta}\right),\left(\mathbb{N}_{0}^{\eta}, \mathbb{N}_{1}^{\eta}\right),\left(\mathbb{B}_{0}^{\eta}, \mathbb{B}_{1}^{\eta}\right),\left(\mathbb{S}_{0}^{\eta}, \mathbb{S}_{1}^{\eta}\right),\left(\mathbb{C}_{0}^{\eta}, \mathbb{C}_{1}^{\eta}\right)\right\}$ for which the inequality $\left(2^{\omega}, 2^{\omega}, \mathbb{A}_{0}, \mathbb{A}_{1}\right) \sqsubseteq\left(X, X, A_{0}, A_{1}\right)$ holds, via a square map,

(3) there is $\left(\mathbb{A}_{0}, \mathbb{A}_{1}\right) \in\left\{\left(\mathbb{N}_{1}^{\eta}, \mathbb{N}_{0}^{\eta}\right),\left(\mathbb{N}_{0}^{\eta}, \mathbb{N}_{1}^{\eta}\right),\left(\mathbb{S}_{0}^{\eta}, \mathbb{S}_{1}^{\eta}\right)\right\}$ such that $\left(2^{\omega}, 2^{\omega}, \mathbb{A}_{0}, \mathbb{A}_{1}\right) \sqsubseteq\left(X, X, A_{0}, A_{1}\right)$. 
Proof. $(1) \Rightarrow(2)$ The proof is partly similar to that of Theorem 3.3 Let $R$ be a potentially closed quasi-acyclic relation containing $A_{0} \cup A_{1}$, and $\left(C_{n}\right)_{n \in \omega}$ be a witness for the fact that $R$ is quasiacyclic. We may assume that $X$ is zero-dimensional (and thus a closed subset of $\omega^{\omega}$ ) and $R$ is closed. In fact, we may assume that $X=\omega^{\omega}$. Indeed, as $A_{0}$ is not separable from $A_{1}$ by a $\operatorname{pot}\left(\Delta\left(D_{\eta}\left(\Sigma_{1}^{0}\right)\right)\right)$ set in $X^{2}$, it is also the case in $\left(\omega^{\omega}\right)^{2}$, which gives $f: 2^{\omega} \rightarrow \omega^{\omega}$. Note that

$$
\mathbb{A}_{0} \cup \mathbb{A}_{1} \subseteq(f \times f)^{-1}\left(A_{0} \cup A_{1}\right) \subseteq(f \times f)^{-1}\left(X^{2}\right),
$$

which implies that $\overline{\mathbb{A}_{0} \cup \mathbb{A}_{1}} \subseteq(f \times f)^{-1}\left(X^{2}\right)$. As $\Delta\left(2^{\omega}\right) \subseteq \mathbb{N}_{0}^{\eta} \cap \overline{\mathbb{S}_{0}^{\eta} \cup \mathbb{S}_{1}^{\eta}}$ and

$$
\left\{(0 \alpha, 1 \alpha) \mid \alpha \in 2^{\omega}\right\} \subseteq \mathbb{B}_{0}^{\eta} \cap \overline{\mathbb{C}_{0}^{\eta} \cup \mathbb{C}_{1}^{\eta}},
$$

the range of $\Delta\left(2^{\omega}\right)$ by $f \times f$ is a subset of $X^{2}$, so that $f$ takes values in $X$. We may also assume that $A_{0}, A_{1}$ are $\Sigma_{1}^{1}$, and that the relation " $(x, y) \in C_{p}$ " is $\Delta_{1}^{1}$ in $(x, y, p)$. By Theorem 4.2, $G_{\eta}$ is a nonempty $\Sigma_{1}^{1}$ relation on $X$ (we denote $G_{\eta}:=G_{\eta, 1}$ and $F_{\eta}^{\varepsilon}:=F_{\eta, 1}^{\varepsilon}$, for simplicity). We also consider $F_{\theta}$ with $F_{\theta}^{\varepsilon}:={\overline{F_{\theta}}}^{\tau_{1}}$. In the sequel, all the closures will refer to the topology $\tau_{1}$, so that, for example,

$$
G_{\eta} \cup A_{0} \cup A_{1} \subseteq \overline{A_{0} \cup A_{1}} \subseteq R=\bigcup_{n \in \omega} C_{n} .
$$

- Let us show that $A_{\epsilon} \cap G_{\eta} \subseteq F_{\eta}^{\mid \text {parity }(\eta)-\epsilon \mid}$ if $\epsilon \in 2$. We argue by induction on $\eta$. If $\eta=1$, then $A_{\epsilon} \cap G_{1} \subseteq A_{\epsilon} \cap \overline{A_{1-\epsilon}} \subseteq F_{1}^{1-\epsilon}$. If $\eta$ is limit, then $A_{\epsilon} \cap G_{\eta} \subseteq A_{\epsilon} \cap \bigcap_{\theta<\eta} F_{\theta}^{\epsilon} \subseteq F_{\eta}^{\epsilon}$. Finally, if $\eta=\theta+1$, then without loss of generality suppose that $\theta$ is even, so that $\eta$ is odd and

$$
A_{\epsilon} \cap G_{\eta} \subseteq A_{\epsilon} \cap \overline{A_{1-\epsilon} \cap G_{\theta}} \subseteq A_{\epsilon} \cap F_{\theta}^{1-\epsilon} .
$$

Note that this last set is contained in $F_{\eta}^{1-\epsilon}$, as required.

So, if $A_{\epsilon} \cap G_{\eta} \neq \emptyset$ for some $\epsilon \in 2$ and $e$ is the correct digit, then $F_{\eta}^{e} \neq \emptyset$. Theorem 3.3 gives $\left(\mathbb{A}_{0}, \mathbb{A}_{1}\right) \in\left\{\left(\mathbb{N}_{1}^{\eta}, \mathbb{N}_{0}^{\eta}\right),\left(\mathbb{B}_{1}^{\eta}, \mathbb{B}_{0}^{\eta}\right),\left(\mathbb{N}_{0}^{\eta}, \mathbb{N}_{1}^{\eta}\right),\left(\mathbb{B}_{0}^{\eta}, \mathbb{B}_{1}^{\eta}\right)\right\}$ for which $\left(2^{\omega}, 2^{\omega}, \mathbb{A}_{0}, \mathbb{A}_{1}\right) \sqsubseteq\left(X, X, A_{0}, A_{1}\right)$, via a square map.

- Thus, in the sequel, we suppose that $G_{\eta} \cap\left(A_{0} \cup A_{1}\right)=\emptyset$. We put

$$
D_{\eta}:=\left\{\left(t_{s}^{0} w, t_{s}^{1} w\right) \in \mathcal{T}^{\eta} \mid s=\emptyset\right\}=\Delta\left(2^{<\omega}\right)
$$

and, for $\theta<\eta$ and $\epsilon \in 2$,

$$
D_{\theta}^{\epsilon}:=\left\{\left(t_{s}^{0} w, t_{s}^{1} w\right) \in \mathcal{T}^{\eta}\left|s \in T_{\eta} \backslash\{\emptyset\} \wedge \varphi(s)=\theta \wedge \operatorname{parity}(|s|)=1-\right| \operatorname{parity}(s(0))-\epsilon \mid\right\},
$$

so that $\left\{D_{\eta}\right\} \cup\left\{D_{\theta}^{\epsilon} \mid \theta<\eta \wedge \epsilon \in 2\right\}$ defines a partition of $\mathcal{T}^{\eta}$.

Case $1 G_{\eta} \not \Delta(X)$.

Let $(x, y) \in G_{\eta} \backslash \Delta(X)$, and $O_{0}, O_{1}$ be disjoint $\Delta_{1}^{0}$ sets with $(x, y) \in O_{0} \times O_{1}$. We can replace $G_{\eta}, A_{0}$ and $A_{1}$ with their intersection with $O_{0} \times O_{1}$ if necessary and assume that they are contained in $O_{0} \times O_{1}$. Let us indicate the differences with the proof of Theorem 3.3 
- Condition (6) is changed as follows:

$$
\text { (6) } U_{s, t} \subseteq\left\{\begin{array}{l}
G_{\eta} \text { if }(s, t) \in D_{\eta} \\
A_{\epsilon} \cap G_{\theta} \text { if }(s, t) \in D_{\theta}^{\epsilon}
\end{array}\right.
$$

- If $(0 \alpha, 1 \beta) \in \mathbb{C}_{\epsilon}^{\eta}$, then there is $\theta<\eta$ such that $(\alpha, \beta) \mid n \in D_{\theta}^{\epsilon}$ if $n \geq n_{0}$. In this case, $\left(U_{(\alpha, \beta) \mid n}\right)_{n>n_{0}}$ is a decreasing sequence of nonempty clopen subsets of $A_{\epsilon} \cap \Omega_{X^{2}}$ with vanishing diameters, so that its intersection is a singleton $\{F(\alpha, \beta)\} \subseteq A_{\epsilon}$, and $(f(0 \alpha), f(1 \beta))=F(\alpha, \beta) \in A_{\epsilon}$.

- So let us prove that the construction is possible. Let $\left(x_{\emptyset}, y_{\emptyset}\right) \in G_{\eta} \cap \Omega_{X^{2}}$. We choose a $\Sigma_{1}^{1}$ subset $U_{\emptyset, \emptyset}$ of $X^{2}$ such that $\left(x_{\emptyset}, y_{\emptyset}\right) \in U_{\emptyset, \emptyset} \subseteq G_{\eta} \cap C_{\Phi(\emptyset, \emptyset)} \cap \Omega_{X^{2}} \cap\left(X_{\emptyset} \times Y_{\emptyset}\right)$, which completes the construction for the length $l=0$. Assume that we have constructed our objects for the sequences of length $l$. Note that $\left(x_{t_{u}^{0} w}, y_{t_{u}^{1} w}\right) \in G_{\varphi(u)} \cap(U \times V) \subseteq G_{\varphi(u q)+1} \cap(U \times V) \subseteq \overline{A_{\epsilon} \cap G_{\varphi(u q)}} \cap(U \times V)$, where $\epsilon$ satisfies $\left(t_{u q}^{0}, t_{u q}^{1}\right) \in D_{\varphi(u q)}^{\epsilon}$. This gives $\left(x_{t_{u}^{0} w 0}, y_{t_{u}^{1} w 1}\right) \in A_{\epsilon} \cap G_{\varphi(u q)} \cap(U \times V) \cap \Omega_{X^{2}}$. If $u=\emptyset$, then $\left(t_{u}^{0} w 1, t_{u}^{1} w 1\right) \in D_{\eta}$, so that $\left(x_{t_{u}^{0} w 1}, y_{t_{u}^{1} w 1}\right) \in U_{t_{u}^{0} w, t_{u}^{1} w} \subseteq G_{\eta}$ and $\left(x_{t_{u}^{0} w 0}, y_{t_{u}^{1} w 1}\right) \in A_{\epsilon}$. As $G_{\eta} \cap\left(A_{0} \cup A_{1}\right)=\emptyset, x_{t_{u}^{0} w 0} \neq x_{t_{u}^{0} w 1}$. Similarly, $y_{t_{u}^{0} w 0} \neq y_{t_{u}^{0} w 1}$. If $u \neq \emptyset$, then we argue as in the proof of Theorem 3.3 to see that $x_{s 0} \neq x_{s 1}$ (and similarly for $y_{s 0}$ and $y_{s 1}$ ).

Case $2 G_{\eta} \subseteq \Delta(X)$.

Let us indicate the differences with the proof of Theorem 3.3 and Case 1. We set

$$
S:=\left\{x \in X \mid(x, x) \in G_{\eta}\right\},
$$

which is a nonempty $\Sigma_{1}^{1}$ set by our assumption. We get $f: 2^{\omega} \rightarrow X$ injective continuous such that $\mathbb{S}_{\epsilon}^{\eta} \subseteq(f \times f)^{-1}\left(A_{\epsilon}\right)$ for each $\epsilon \in 2$. In this case, $A_{0} \cap S^{2}$ and $A_{1} \cap S^{2}$ are irreflexive.

$(2) \Rightarrow(3)$ Note that $\left(2^{\omega}, 2^{\omega}, \mathbb{N}_{0}^{\eta}, \mathbb{N}_{1}^{\eta}\right) \sqsubseteq\left(2^{\omega}, 2^{\omega}, \mathbb{B}_{0}^{\eta}, \mathbb{B}_{1}^{\eta}\right)$ and $\left(2^{\omega}, 2^{\omega}, \mathbb{S}_{0}^{\eta}, \mathbb{S}_{1}^{\eta}\right) \sqsubseteq\left(2^{\omega}, 2^{\omega}, \mathbb{C}_{0}^{\eta}, \mathbb{C}_{1}^{\eta}\right)$, with witnesses $\alpha \rightarrow 0 \alpha$ and $\beta \rightarrow 1 \beta$.

(3) $\Rightarrow$ (1) This comes from Lemmas 3.1 and 4.1

Proposition 4.4 Let $\eta \geq 1$ be a countable ordinal.

(a) If $\eta$ is a successor ordinal, then the pairs $\left(\mathbb{N}_{1}^{\eta}, \mathbb{N}_{0}^{\eta}\right),\left(\mathbb{B}_{1}^{\eta}, \mathbb{B}_{0}^{\eta}\right),\left(\mathbb{N}_{0}^{\eta}, \mathbb{N}_{1}^{\eta}\right),\left(\mathbb{B}_{0}^{\eta}, \mathbb{B}_{1}^{\eta}\right),\left(\mathbb{S}_{0}^{\eta}, \mathbb{S}_{1}^{\eta}\right)$ and $\left(\mathbb{C}_{0}^{\eta}, \mathbb{C}_{1}^{\eta}\right)$ are incomparable for the square reduction.

(b) If $\eta$ is a limit ordinal, then $\left(2^{\omega}, 2^{\omega}, \mathbb{S}_{0}^{\eta}, \mathbb{S}_{1}^{\eta}\right) \sqsubseteq\left(2^{\omega}, 2^{\omega}, \mathbb{N}_{1}^{\eta}, \mathbb{N}_{0}^{\eta}\right),\left(2^{\omega}, 2^{\omega}, \mathbb{N}_{0}^{\eta}, \mathbb{N}_{1}^{\eta}\right)$ and

$$
\left(2^{\omega}, 2^{\omega}, \mathbb{C}_{0}^{\eta}, \mathbb{C}_{1}^{\eta}\right) \sqsubseteq\left(2^{\omega}, 2^{\omega}, \mathbb{B}_{1}^{\eta}, \mathbb{B}_{0}^{\eta}\right),\left(2^{\omega}, 2^{\omega}, \mathbb{B}_{0}^{\eta}, \mathbb{B}_{1}^{\eta}\right),
$$

via a square map, and the pairs $\left(\mathbb{S}_{0}^{\eta}, \mathbb{S}_{1}^{\eta}\right)$ and $\left(\mathbb{C}_{0}^{\eta}, \mathbb{C}_{1}^{\eta}\right)$ are incomparable for the square reduction.

Proof. (a) We set, for $\theta \leq \eta, C_{\theta}:=\bigcup_{\varphi(s) \geq \theta} \operatorname{Gr}\left(f_{s}\right)$.

Claim. Let $\theta \leq \eta$. Then $C_{\theta}$ is a closed relation on $2^{\omega}$.

Indeed, this is inspired by the proof of Theorem 2.3 in [L2]. 
We first show that $C^{l}:=\bigcup_{s \in \omega \leq l, \varphi(s) \geq \theta} \operatorname{Gr}\left(f_{s}\right)$ is closed, by induction on $l \in \omega$. This is clear for $l=0$. Assume that the statement is true for $l$. Note that $C^{l+1}=C^{l} \cup \bigcup_{s \in \omega^{l+1}, \varphi(s) \geq \theta} \operatorname{Gr}\left(f_{s}\right)$. Let $p_{m} \in C^{l+1}$ such that $\left(p_{m}\right)_{m \in \omega}$ converges to $p$. By induction assumption, we may assume that, for each $m$, there is $\left(s_{m}, n_{m}\right) \in \omega^{l} \times \omega$ such that $\varphi\left(s_{m} n_{m}\right) \geq \theta$ and $p_{m} \in \operatorname{Gr}\left(f_{s_{m} n_{m}}\right)$. As the $\operatorname{Gr}\left(f_{s n}\right)$ 's are closed, we may assume that there is $i \leq l$ such that the sequence $\left(\left(s_{m} n_{m}\right) \mid i\right)_{m \in \omega}$ is constant and the sequence $\left(\left(s_{m} n_{m}\right)(i)\right)_{m \in \omega}$ tends to infinity. This implies that $p \in \operatorname{Gr}\left(f_{\left(s_{0} n_{0}\right) \mid i} \subseteq \subseteq C^{l+1}\right.$, which is therefore closed.

Now let $p_{m} \in C_{\theta}$ such that $\left(p_{m}\right)_{m \in \omega}$ converges to $p$. The previous fact implies that we may assume that, for each $m$, there is $s_{m}^{\prime}$ such that $\varphi\left(s_{m}^{\prime}\right) \geq \theta$ and $p_{m} \in \operatorname{Gr}\left(f_{s_{m}^{\prime}}\right)$, and that the sequence $\left(\left|s_{m}^{\prime}\right|\right)_{m \in \omega}$ tends to infinity. Note that there is $l$ such that the set of $s_{m}^{\prime}(l)$ 's is infinite. Indeed, assume, towards a contradiction, that this is not the case. Then $\left\{s \in T_{\eta} \mid \exists m \in \omega s \subseteq s_{m}^{\prime}\right\}$ is an infinite finitely branching subtree of $T_{\eta}$. By König's lemma, it has an infinite branch, which contradicts the wellfoundedness of $T_{\eta}$. So we may assume that there is $l$ such that the sequence $\left(s_{m}^{\prime} \mid l\right)_{m \in \omega}$ is constant and the sequence $\left(s_{m}^{\prime}(l)\right)_{m \in \omega}$ tends to infinity. This implies that $p \in \operatorname{Gr}\left(f_{s_{0}^{\prime} \mid l}\right) \subseteq C_{\theta}$.

- By Lemma 3.1, $\mathbb{N}_{0}^{\eta}$ is not separable from $\mathbb{N}_{1}^{\eta}$ by a $\operatorname{pot}\left(D_{\eta}\left(\boldsymbol{\Sigma}_{1}^{0}\right)\right)$ set, and, by Lemma 4.1, $\mathbb{S}_{0}^{\eta}$ is not separable from $\mathbb{S}_{1}^{\eta}$ by a $\operatorname{pot}\left(\Delta\left(D_{\eta}\left(\Sigma_{1}^{0}\right)\right)\right)$ set.

- Let us show that $\mathbb{N}_{0}^{\eta}$ is separable from $\mathbb{N}_{1}^{\eta}$ by a $\check{D}_{\eta}\left(\Sigma_{1}^{0}\right)$ set. In fact, it is enough to see that $\mathbb{N}_{0}^{\eta} \in \check{D}_{\eta}\left(\boldsymbol{\Sigma}_{1}^{0}\right)$ if $\eta$ is odd and $\mathbb{N}_{1}^{\eta} \in D_{\eta}\left(\boldsymbol{\Sigma}_{1}^{0}\right)$ if $\eta$ is even. If $\eta$ is odd, then

$$
\mathbb{N}_{0}^{\eta}=\bigcup_{s \in T_{\eta}, \varphi(s) \text { odd }} \operatorname{Gr}\left(f_{s}\right)=C_{\eta} \cup \bigcup_{\theta<\eta, \theta \text { odd }} C_{\theta} \backslash C_{\theta+1} .
$$

We set, for $\theta<\eta, O_{\theta}:=\neg C_{\theta+1}$, which defines an increasing sequence of open relations on $2^{\omega}$ with $\mathbb{N}_{0}^{\eta}=\neg O_{\eta-1} \cup \bigcup_{\theta<\eta, \theta}$ odd $O_{\theta} \backslash O_{\theta-1}$. Thus $\mathbb{N}_{0}^{\eta} \in \check{D}_{\eta}\left(\Sigma_{1}^{0}\right)$. Similarly, if $\eta$ is even, then

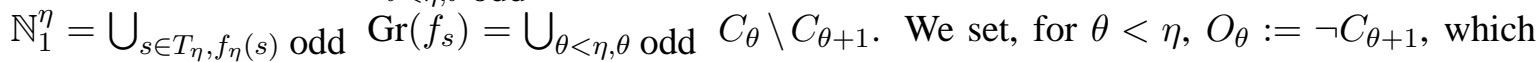
defines an increasing sequence of open relations on $2^{\omega}$ with $\mathbb{N}_{1}^{\eta}=\bigcup_{\theta<\eta, \theta \text { odd }} O_{\theta} \backslash O_{\theta-1}$. Thus $\mathbb{N}_{1}^{\eta} \in D_{\eta}\left(\Sigma_{1}^{0}\right)$. This shows that $\left(2^{\omega}, 2^{\omega}, \mathbb{N}_{1}^{\eta}, \mathbb{N}_{0}^{\eta}\right)$ is not $\sqsubseteq$-below $\left(2^{\omega}, 2^{\omega}, \mathbb{N}_{0}^{\eta}, \mathbb{N}_{1}^{\eta}\right)$, and consequently that $\left(2^{\omega}, 2^{\omega}, \mathbb{N}_{0}^{\eta}, \mathbb{N}_{1}^{\eta}\right)$ is not $\sqsubseteq$-below $\left(2^{\omega}, 2^{\omega}, \mathbb{N}_{1}^{\eta}, \mathbb{N}_{0}^{\eta}\right)$.

- Let us show that $\mathbb{S}_{\varepsilon}^{\eta}$ is separable from $\mathbb{S}_{1-\varepsilon}^{\eta}$ by a $\check{D}_{\eta}\left(\boldsymbol{\Sigma}_{1}^{0}\right)$ set if $\varepsilon \in 2$. We set, for $\theta \leq \eta$,

As in the claim, $\left(C_{\theta}^{\varepsilon}\right)_{\theta \leq \eta}$ is a decreasing sequence of closed sets. 
Note that

$$
\begin{aligned}
& \mathbb{S}_{\varepsilon}^{\eta}=\bigcup_{s \in T_{\eta} \backslash\{\emptyset\}, \text { parity }(|s|)=1-|\operatorname{parity}(s(0))-\varepsilon|} \operatorname{Gr}\left(f_{s}\right) \\
& =\bigcup_{s \in T_{\eta} \backslash\{\emptyset\},|\operatorname{parity}(\varphi(s))-\operatorname{parity}(\eta)|=1-|\operatorname{parity}(s(0))-\varepsilon|} \operatorname{Gr}\left(f_{s}\right) \\
& =\bigcup_{s \in T_{\eta} \backslash\{\emptyset\} \text {, parity }(s(0))=|1-||\operatorname{parity}(\varphi(s))-\operatorname{parity}(\eta)|-\varepsilon \|} \operatorname{Gr}\left(f_{s}\right) \\
& =\bigcup_{\theta<\eta, \varphi(s)=\theta} \bigcup_{\text {parity }(s(0))=|1-||\operatorname{parity}(\theta)-\operatorname{parity}(\eta)|-\varepsilon||} \operatorname{Gr}\left(f_{s}\right) \\
& =\bigcup_{\theta<\eta}\left(\bigcup_{\varphi(s) \geq \theta \text {, parity }(s(0))=|1-| \mid \text { parity }(\theta)-\text { parity }(\eta)|-\varepsilon| \mid} \operatorname{Gr}\left(f_{s}\right)\right) \backslash \\
& \left(\bigcup_{\varphi(s) \geq \theta+1, \operatorname{parity}(s(0))=|1-||\operatorname{parity}(\theta)-\operatorname{parity}(\eta)|-\varepsilon||} \operatorname{Gr}\left(f_{s}\right)\right) \\
& =\bigcup_{\theta<\eta} C_{\theta}^{1-|| \operatorname{parity}(\theta)-\operatorname{parity}(\eta)|-\varepsilon|} \backslash C_{\theta+1}^{1-|| \operatorname{parity}(\theta)-\operatorname{parity}(\eta)|-\varepsilon|} \text {. }
\end{aligned}
$$

Assume first that $\eta=\theta_{0}+1$ is a successor ordinal. We define an increasing sequence $\left(O_{\theta}\right)_{\theta<\eta}$ of open sets as follows:

$$
O_{\theta}:=\left\{\begin{array}{l}
\neg\left(C_{\theta+1}^{1-\varepsilon} \cup C_{\theta}^{\varepsilon}\right) \text { if } \theta<\theta_{0}, \\
\neg C_{\theta}^{\varepsilon} \text { if } \theta=\theta_{0},
\end{array}\right.
$$

so that $D:=\neg D\left(\left(O_{\theta}\right)_{\theta<\eta}\right) \in \check{D}_{\eta}\left(\Sigma_{1}^{0}\right)$.

We now check that $D$ separates $\mathbb{S}_{\varepsilon}^{\eta}$ from $\mathbb{S}_{1-\varepsilon}^{\eta}$. If $\theta<\eta$ has a parity opposite to that of $\eta$, then either $\theta=\theta_{0}$ and $C_{\theta}^{\varepsilon} \backslash C_{\theta+1}^{\varepsilon} \subseteq C_{\theta_{0}}^{\varepsilon} \subseteq \neg\left(\bigcup_{\theta^{\prime}<\eta} O_{\theta^{\prime}}\right) \subseteq D$. Or $\theta<\theta_{0}, \theta+1<\theta_{0}<\eta$ has the same parity as $\eta$, and $C_{\theta}^{\varepsilon} \backslash C_{\theta+1}^{\varepsilon} \subseteq O_{\theta+1} \backslash\left(\bigcup_{\theta^{\prime} \leq \theta} O_{\theta^{\prime}}\right) \subseteq D$. If now $\theta<\eta$ has the same parity as $\eta$, then $C_{\theta}^{1-\varepsilon} \backslash C_{\theta+1}^{1-\varepsilon} \subseteq O_{\theta} \backslash\left(\bigcup_{\theta^{\prime}<\theta} O_{\theta^{\prime}}\right) \subseteq D$. Thus $\mathbb{S}_{\varepsilon}^{\eta} \subseteq D$. Similarly, $\mathbb{S}_{1-\varepsilon}^{\eta} \subseteq \neg D$. If $\eta$ is a limit ordinal, then we set $O_{\theta}:=\neg\left(C_{\theta+1}^{1-\varepsilon} \cup C_{\theta}^{\varepsilon}\right)$ and argue similarly. This shows that $\left(2^{\omega}, 2^{\omega}, \mathbb{N}_{\varepsilon}^{\eta}, \mathbb{N}_{1-\varepsilon}^{\eta}\right)$ is not $\sqsubseteq$-below $\left(2^{\omega}, 2^{\omega}, \mathbb{S}_{0}^{\eta}, \mathbb{S}_{1}^{\eta}\right)$ for each $\varepsilon \in 2$.

- Let us prove that $\left(2^{\omega}, 2^{\omega}, \mathbb{S}_{0}^{\eta}, \mathbb{S}_{1}^{\eta}\right)$ is not $\sqsubseteq$-below $\left(2^{\omega}, 2^{\omega}, \mathbb{N}_{\varepsilon}^{\eta}, \mathbb{N}_{1-\varepsilon}^{\eta}\right)$ if $\varepsilon \in 2$ and $\eta$ is a successor ordinal. Let us do it for $\varepsilon=0$, the other case being similar. We argue by contradiction, which gives $f, g$ injective continuous with $\mathbb{S}_{\varepsilon}^{\eta} \subseteq(f \times g)^{-1}\left(\mathbb{N}_{\varepsilon}^{\eta}\right)$ for each $\varepsilon \in 2$. We set, for $\theta<\eta$ and $\varepsilon \in 2$,

$$
U_{\theta}^{\varepsilon}:=\underset{\theta \leq \theta^{\prime}<\eta, \varphi(s)=\theta^{\prime}, \operatorname{parity}(s(0))=|1-|\left|\operatorname{parity}\left(\theta^{\prime}\right)-\operatorname{parity}(\eta)\right|-\varepsilon||}{\bigcup} \operatorname{Gr}\left(f_{s}\right) .
$$

Note that the sequence $\left(U_{\theta}^{\varepsilon}\right)_{\theta<\eta}$ is decreasing, $\mathbb{S}_{\varepsilon}^{\eta}=U_{0}^{\varepsilon}$,

$$
\overline{U_{\theta}^{0} \cup U_{\theta}^{1}}=C_{\theta}^{0} \cup C_{\theta}^{1}=U_{\theta}^{0} \cup U_{\theta}^{1} \cup \Delta\left(2^{\omega}\right)=C_{\theta},
$$

and $C_{\theta+1}^{0} \cup C_{\theta+1}^{1}=\overline{U_{\theta}^{0}} \cap \overline{U_{\theta}^{1}}$ if $\theta<\eta$ since

$$
\overline{U_{\theta}^{\varepsilon}}=C_{\theta+1}^{0} \cup C_{\theta+1}^{1} \cup \bigcup_{\varphi(s)=\theta, \operatorname{parity}(s(0))=|1-||\operatorname{parity}(\theta)-\operatorname{parity}(\eta)|-\varepsilon||} \operatorname{Gr}\left(f_{s}\right),
$$

as in the claim. Let us prove that $U_{\theta}^{0} \cup U_{\theta}^{1} \subseteq(f \times g)^{-1}\left(C_{\theta}\right)$ if $\theta<\eta$. We argue by induction on $\theta$, and the result is clear for $\theta=0$. If $\theta=\theta^{\prime}+1$ is a successor ordinal, then

$$
U_{\theta}^{0} \cup U_{\theta}^{1} \subseteq C_{\theta}^{0} \cup C_{\theta}^{1}=\overline{U_{\theta^{\prime}}^{0}} \cap \overline{U_{\theta^{\prime}}^{1}} \subseteq(f \times g)^{-1}\left(\overline{\mathbb{N}_{0}^{\eta} \cap C_{\theta^{\prime}}} \cap \overline{\mathbb{N}_{1}^{\eta} \cap C_{\theta^{\prime}}}\right) \subseteq(f \times g)^{-1}\left(C_{\theta}\right) .
$$

If $\theta$ is a limit ordinal, then $U_{\theta}^{0} \cup U_{\theta}^{1} \subseteq \bigcap_{\theta^{\prime}<\theta}\left(U_{\theta^{\prime}}^{0} \cup U_{\theta^{\prime}}^{1}\right) \subseteq(f \times g)^{-1}\left(\bigcap_{\theta^{\prime}<\theta} C_{\theta^{\prime}}\right)=(f \times g)^{-1}\left(C_{\theta}\right)$. This implies that $C_{\eta}^{0} \cup C_{\eta}^{1} \subseteq(f \times g)^{-1}\left(C_{\eta}\right)$. In particular, $\Delta\left(2^{\omega}\right)$ is sent into itself by $f \times g$ and $f=g$. As $\eta=\theta+1$ is a successor ordinal, $U_{\theta}^{0} \subseteq(f \times f)^{-1}\left(\mathbb{N}_{0}^{\eta} \cap C_{\theta}\right) \subseteq(f \times f)^{-1}\left(\Delta\left(2^{\omega}\right)\right)$, which contradicts the injectivity of $f$. 
- So we proved that $\mathcal{A}:=\left\{\left(\mathbb{N}_{1}^{\eta}, \mathbb{N}_{0}^{\eta}\right),\left(\mathbb{N}_{0}^{\eta}, \mathbb{N}_{1}^{\eta}\right),\left(\mathbb{S}_{0}^{\eta}, \mathbb{S}_{1}^{\eta}\right)\right\}$ is a $\sqsubseteq$-antichain if $\eta$ is a successor ordinal. For the same reasons, $\mathcal{B}:=\left\{\left(\mathbb{B}_{1}^{\eta}, \mathbb{B}_{0}^{\eta}\right),\left(\mathbb{B}_{0}^{\eta}, \mathbb{B}_{1}^{\eta}\right),\left(\mathbb{C}_{0}^{\eta}, \mathbb{C}_{1}^{\eta}\right)\right\}$ is a $\sqsubseteq$-antichain if $\eta$ is a successor ordinal. Moreover, no pair in $\mathcal{A}$ is below a pair in $\mathcal{B}$ for the square reduction since $\Delta\left(2^{\omega}\right) \subseteq \mathbb{N}_{0}^{\eta} \cap \overline{\mathbb{S}_{0}^{\eta} \cup \mathbb{S}_{1}^{\eta}}$ and the element of the pairs in $\mathcal{B}$ are contained in the clopen set $N_{0} \times N_{1}$.

It remains to prove that we cannot find $(\mathbb{A}, \mathbb{B}),\left(\mathbb{A}^{\prime}, \mathbb{B}^{\prime}\right) \in \mathcal{A}$ and a continuous injection $f: 2^{\omega} \rightarrow 2^{\omega}$ such that $G_{\mathbb{A}} \subseteq(f \times f)^{-1}\left(\mathbb{A}^{\prime}\right)$ and $G_{\mathbb{B}} \subseteq(f \times f)^{-1}\left(\mathbb{B}^{\prime}\right)$. We argue by contradiction. If $(\mathbb{A}, \mathbb{B}) \neq\left(\mathbb{A}^{\prime}, \mathbb{B}^{\prime}\right)$ and $\varepsilon \in 2$, then we define continuous injections $f_{\varepsilon}: 2^{\omega} \rightarrow 2^{\omega}$ by $f_{\varepsilon}(\alpha):=f(\varepsilon \alpha)$. Note that $f_{0} \times f_{1}$ reduces $(\mathbb{A}, \mathbb{B})$ to $\left(\mathbb{A}^{\prime}, \mathbb{B}^{\prime}\right)$, which contradicts the fact that $\mathcal{A}$ is a $\sqsubseteq$-antichain. Thus $(\mathbb{A}, \mathbb{B})=\left(\mathbb{A}^{\prime}, \mathbb{B}^{\prime}\right)$, and $(\mathbb{A}, \mathbb{B})=\left(\mathbb{S}_{0}^{\eta}, \mathbb{S}_{1}^{\eta}\right)$ by Proposition 3.4. As in the proof of Proposition 3.4 $\varphi(s) \leq \varphi(v)$. If $\alpha \in 2^{\omega}$, then $(0 \alpha, 1 \alpha)$ is the limit of $\left(0 t_{p_{k}}^{0} \gamma_{k}, 1 t_{p_{k}}^{1} \gamma_{k}\right)$. Note that $\left(f\left(0 t_{p_{k}}^{0} \gamma_{k}\right), f\left(1 t_{p_{k}}^{1} \gamma_{k}\right)\right)=\left(t_{v_{k}}^{0} \gamma_{k}^{\prime}, t_{v_{k}}^{1} \gamma_{k}^{\prime}\right)$ and $\varphi\left(p_{k}\right) \leq \varphi\left(v_{k}\right)$. As $\left(\varphi\left(p_{k}\right)\right)_{k \in \omega}$ is cofinal in $\varphi(\emptyset)=\eta$, so is $\left(\varphi\left(v_{k}\right)\right)_{k \in \omega}$. This implies that $(f(0 \alpha), f(1 \alpha)) \in \Delta\left(2^{\omega}\right)$, which contradicts the injectivity of $f$.

(b) Let us prove that $\left(2^{\omega}, 2^{\omega}, \mathbb{S}_{0}^{\eta}, \mathbb{S}_{1}^{\eta}\right) \sqsubseteq\left(2^{\omega}, 2^{\omega}, \mathbb{N}_{\varepsilon}^{\eta}, \mathbb{N}_{1-\varepsilon}^{\eta}\right)$ with a square map if $\varepsilon \in 2$. Let us do it for $\varepsilon=0$, the other case being similar. We construct a map $\phi: 2^{<\omega} \rightarrow 2^{<\omega}$ satisfying the following:

(1) $\forall l \in \omega \quad \exists k_{l} \in \omega \quad \phi\left[2^{l}\right] \subseteq 2^{k_{l}}$

(2) $\phi(s) \varsubsetneqq \phi(s \varepsilon)$

(3) $\phi(s 0) \neq \phi(s 1)$

(4) $\forall s \in T_{\eta} \backslash\{\emptyset\} \quad($ parity $(|s|)=1-|\operatorname{parity}(s(0))-\varepsilon|) \Rightarrow \exists v_{s} \in T_{\eta}$ parity $\left(\left|v_{s}\right|\right)=\varepsilon \wedge$

(a) $\forall w \in 2^{<\omega} \exists w^{\prime} \in 2^{<\omega} \quad\left(\phi\left(t_{s}^{0} w\right), \phi\left(t_{s}^{1} w\right)\right)=\left(t_{v_{s}}^{0} w^{\prime}, t_{v_{s}}^{1} w^{\prime}\right)$

(b) $\varphi(s) \leq \varphi\left(v_{s}\right)$

Assume that this is done. Then the map $f: \alpha \mapsto \lim _{n \rightarrow \infty} \phi(\alpha \mid n)$ is as desired. So let us check that the construction of $\phi$ is possible. We construct $\phi(s)$ by induction on the length of $s$.

- We set $k_{0}:=0$ and $\phi(\emptyset):=\emptyset$.

- Note that $<0>_{\eta}=1$ and $\left(t_{0}^{0}, t_{0}^{1}\right)=(0,1)$. As $\eta \geq 1$ is limit, $\varphi(1)>\varphi(0)$ are odd ordinals, so that $\varphi(10) \geq \varphi(0)$ is an even ordinal. We set $k_{1}:=<10>_{\eta}, \phi(\varepsilon):=t_{10}^{\varepsilon}$ and $v_{0}:=10$. This completes the construction of $\phi\left[2^{1}\right]$, and our conditions are satisfied since $k_{1}>0$.

- We next want to construct $\phi(s)$ for $s \in 2^{l+1}$, with $l \geq 1$, assuming that we have constructed $\phi(s)$ if $|s| \leq l$. Note that there is exactly one sequence $u$ such that $\left(t_{u}^{0}, t_{u}^{1}\right) \in 2^{l+1}$. We first define simultaneously $\phi\left(t_{u}^{0}\right)$ and $\phi\left(t_{u}^{1}\right)$, and then extend the definition to the other sequences in $2^{l+1}$.

If $|u| \geq 2$, then there are $u_{0} \in \omega^{<\omega}$ and $w \in 2^{<\omega}$ such that $t_{u}^{\varepsilon}=t_{u_{0}}^{\varepsilon} w \varepsilon$. By condition (4), $\left(\phi\left(t_{u_{0}}^{0} w\right), \phi\left(t_{u_{0}}^{0} w\right)\right)=\left(t_{v}^{0} w^{\prime}, t_{v}^{1} w^{\prime}\right)$ for some $v \in \omega^{<\omega}$ and $w^{\prime} \in 2^{<\omega}$. Let $q \in \omega$ such that $w^{\prime} \subseteq \psi(q)$ and $\varphi(u) \leq \varphi(v q)$. We can find such a $q$ because if $\varphi(v)=\nu+1$, then $\varphi(v q)=\nu$, but $\varphi(u)<\varphi\left(u_{0}\right) \leq \nu+1$ so that $\varphi(u) \leq \nu$. If $\varphi(v)$ is limit, then $(\varphi(v q))_{q \in \omega}$ is cofinal in $\varphi(v)$ and $\varphi(u)<\varphi\left(u_{0}\right) \leq \varphi(v)$. We set $\phi\left(t_{u_{0}}^{\varepsilon} w \varepsilon\right):=t_{v q}^{\varepsilon}$. By definition, there is $N \in \omega$ such that $t_{v q}^{\varepsilon}=t_{v}^{\varepsilon} w^{\prime} 0^{N} \varepsilon$. We set $\phi(s \varepsilon):=\phi(s) 0^{N} \varepsilon$, for any $s \in 2^{l}$. Conditions (1)-(3) clearly hold. So let us check condition (4). First note that $\left(\phi\left(t_{u}^{0}\right), \phi\left(t_{u}^{1}\right)\right)=\left(t_{v q}^{0}, t_{v q}^{1}\right)$ by definition, so that (4) holds for $u$ since $|u|-\left|u_{0}\right|=|v q|-|v|=1$. 
Suppose now that there are $u_{1} \in \omega^{<\omega}, z \in 2^{<\omega}$ and $e \in 2$ such that $(s, t)=\left(t_{u_{1}}^{0} z e, t_{u_{1}}^{1} z e\right)$. By the induction hypothesis, $\left(\phi\left(t_{u_{1}}^{0} z e\right), \phi\left(t_{u_{1}}^{1} z e\right)\right)=\left(\phi\left(t_{u_{1}}^{0} z\right) 0^{N} e, \phi\left(t_{u_{1}}^{1} z\right) 0^{N} e\right)=\left(t_{v_{u_{1}}}^{0} z^{\prime} 0^{N} e, t_{v_{u_{1}}}^{1} z^{\prime} 0^{N} e\right)$. Thus conditions (4) is checked.

Otherwise, $|u|=1$ and $u=\left\langle p>\right.$ for some $p \in \omega \backslash\{0\}$. Let $\left.w:=t_{u}^{0}\right| l$. Note there are infinitely many $q$ 's such that $\phi(w) \subseteq \psi(q)$. As $\eta$ is a limit ordinal, $(\varphi(q))_{q \in \omega}$ is strictly increasing. Thus $q$ can be chosen so that $\varphi(p) \leq \varphi(q)$. If $p$ is odd, then we set $\phi\left(t_{u}^{\varepsilon}\right):=t_{<q>}^{\varepsilon}$. If $p$ is even, then we set $\phi\left(t_{u}^{\varepsilon}\right):=t_{q 0}^{\varepsilon}$. Let $w^{0}$ and $w^{1}$ be the sequences such that $\phi\left(t_{u}^{\varepsilon}\right)=\phi(w) w^{\varepsilon} \varepsilon$. Note that they are different if $p$ is even. As in the previous case, we define $\phi(s \varepsilon):=\phi(s) w^{\varepsilon} \varepsilon$, for any $s \in 2^{l}$. Notice how the choice of $w^{\varepsilon}$ only depends on the last coordinate of $s \varepsilon$. The conditions are verified as before for $\left(\phi\left(t_{u}^{0}\right), \phi\left(t_{u}^{1}\right)\right)$. For the other cases,

$$
\left(\phi\left(t_{u_{1}}^{0} z e\right), \phi\left(t_{u_{1}}^{1} z e\right)\right)=\left(\phi\left(t_{u_{1}}^{0} z\right) w^{e} e, \phi\left(t_{u_{1}}^{1} z\right) w^{e} e\right)=\left(t_{v_{u_{1}}}^{0} w^{\prime} w^{e} e, t_{v_{u_{1}}}^{1} w^{\prime} w^{e} e\right)
$$

by the induction hypothesis. So the conditions are checked.

It remains to note that $\left(2^{\omega}, 2^{\omega}, \mathbb{C}_{0}^{\eta}, \mathbb{C}_{1}^{\eta}\right) \sqsubseteq\left(2^{\omega}, 2^{\omega}, \mathbb{B}_{\varepsilon}^{\eta}, \mathbb{B}_{1-\varepsilon}^{\eta}\right)$ with a square map if $\varepsilon \in 2$, with witness $\varepsilon \alpha \mapsto \varepsilon f(\alpha)$.

\section{Consequences}

Corollary 4.5 Let $\eta \geq 1$ be a countable ordinal, $X$ be a Polish space, and $A, B$ be disjoint analytic relations on $X$ such that $A \cup B$ is contained in a potentially closed s-acyclic or locally countable relation. Then exactly one of the following holds:

(a) the set $A$ is separable from $B$ by a pot $\left(\Delta\left(D_{\eta}\left(\Sigma_{1}^{0}\right)\right)\right)$ set,

(b) there is $\left(\mathbb{A}_{0}, \mathbb{A}_{1}\right) \in\left\{\left(\mathbb{N}_{1}^{\eta}, \mathbb{N}_{0}^{\eta}\right),\left(\mathbb{N}_{0}^{\eta}, \mathbb{N}_{1}^{\eta}\right),\left(\mathbb{S}_{0}^{\eta}, \mathbb{S}_{1}^{\eta}\right)\right\}$ with $\left(2^{\omega}, 2^{\omega}, \mathbb{A}_{0}, \mathbb{A}_{1}\right) \sqsubseteq(X, X, A, B)$.

Proof. By Lemmas 3.1 and 4.1, (a) and (b) cannot hold simultaneously. So assume that (a) does not hold. By Lemma 2.2, we may assume that $A \cup B$ is contained in a potentially closed quasi-acyclic relation. It remains to apply Theorem 4.3 .

Corollary 4.6 Let $\eta \geq 1$ be a countable ordinal, $X, Y$ be Polish spaces, and $A, B$ be disjoint analytic subsets of $X \times Y$ such that $A \cup B$ is contained in a potentially closed locally countable set. Then exactly one of the following holds:

(a) the set $A$ is separable from $B$ by a pot $\left(\Delta\left(D_{\eta}\left(\boldsymbol{\Sigma}_{1}^{0}\right)\right)\right)$ set,

(b) there is $\left(\mathbb{A}_{0}, \mathbb{A}_{1}\right) \in\left\{\left(\mathbb{N}_{1}^{\eta}, \mathbb{N}_{0}^{\eta}\right),\left(\mathbb{N}_{0}^{\eta}, \mathbb{N}_{1}^{\eta}\right),\left(\mathbb{S}_{0}^{\eta}, \mathbb{S}_{1}^{\eta}\right)\right\}$ with $\left(2^{\omega}, 2^{\omega}, \mathbb{A}_{0}, \mathbb{A}_{1}\right) \sqsubseteq(X, X, A, B)$.

Proof. As in the proof of Corollary 4.5, (a) and (b) cannot hold simultaneously. Then we argue as in the proof of Corollary 3.7. $A^{\prime} \cup B^{\prime}$ is contained in a potentially closed locally countable relation, and $A^{\prime}$ is not separable from $B^{\prime}$ by a $\operatorname{pot}\left(\Delta\left(D_{\eta}\left(\boldsymbol{\Sigma}_{1}^{0}\right)\right)\right)$ set. Corollary 4.5 gives $f^{\prime}, g^{\prime}: 2^{\omega} \rightarrow Z$. 
Corollary 4.7 Let $\eta \geq 1$ be a countable ordinal, $X$ be a Polish space, and $A, B$ be disjoint analytic relations on $X$. The following are equivalent:

(1) there is a potentially closed s-acyclic relation $R \in \Sigma_{1}^{1}$ such that $A \cap R$ is not separable from $B \cap R$ by $a \operatorname{pot}\left(\Delta\left(D_{\eta}\left(\Sigma_{1}^{0}\right)\right)\right)$ set,

(2) there is a potentially closed locally countable relation $R \in \Sigma_{1}^{1}$ such that $A \cap R$ is not separable from $B \cap R$ by a pot $\left(\Delta\left(D_{\eta}\left(\Sigma_{1}^{0}\right)\right)\right)$ set,

(3) there is $\left(\mathbb{A}_{0}, \mathbb{A}_{1}\right) \in\left\{\left(\mathbb{N}_{1}^{\eta}, \mathbb{N}_{0}^{\eta}\right),\left(\mathbb{N}_{0}^{\eta}, \mathbb{N}_{1}^{\eta}\right),\left(\mathbb{S}_{0}^{\eta}, \mathbb{S}_{1}^{\eta}\right)\right\}$ with $\left(2^{\omega}, 2^{\omega}, \mathbb{A}_{0}, \mathbb{A}_{1}\right) \sqsubseteq(X, X, A, B)$,

(4) there is $\left(\mathbb{A}_{0}, \mathbb{A}_{1}\right) \in\left\{\left(\mathbb{N}_{1}^{\eta}, \mathbb{N}_{0}^{\eta}\right),\left(\mathbb{B}_{1}^{\eta}, \mathbb{B}_{0}^{\eta}\right),\left(\mathbb{N}_{0}^{\eta}, \mathbb{N}_{1}^{\eta}\right),\left(\mathbb{B}_{0}^{\eta}, \mathbb{B}_{1}^{\eta}\right),\left(\mathbb{S}_{0}^{\eta}, \mathbb{S}_{1}^{\eta}\right),\left(\mathbb{C}_{0}^{\eta}, \mathbb{C}_{1}^{\eta}\right)\right\}$ such that the inequality $\left(2^{\omega}, 2^{\omega}, \mathbb{A}_{0}, \mathbb{A}_{1}\right) \sqsubseteq(X, X, A, B)$ holds, via a square map.

Proof. (1) $\Rightarrow(3),(4)$ and (2) $\Rightarrow(3),(4)$ This is a consequence of Corollary 4.5 and its proof.

(4) $\Rightarrow$ (1) By the remarks before Lemma 3.1 $\mathbb{N}_{0}^{\eta} \cup \mathbb{N}_{1}^{\eta}$ has s-acyclic levels. This implies that $\mathbb{N}_{0}^{\eta} \cup \mathbb{N}_{1}^{\eta}$ and $\mathbb{S}_{0}^{\eta} \cup \mathbb{S}_{1}^{\eta}$ are s-acyclic. As $\mathbb{N}_{0}^{\eta} \cup \mathbb{N}_{1}^{\eta}$ is antisymmetric, $\mathbb{B}_{0}^{\eta} \cup \mathbb{B}_{1}^{\eta}$ and $\mathbb{C}_{0}^{\eta} \cup \mathbb{C}_{1}^{\eta}$ are s-acyclic too, by Lemma 3.8. Thus we can take $R:=(f \times f)\left[\mathbb{A}_{0} \cup \mathbb{A}_{1}\right]$ since the s-acyclicity is preserved by images by the square of an injection, and by Lemmas 3.1 and 4.1 .

(3),(4) $\Rightarrow(2)$ We can take $R:=(f \times f)\left[\mathbb{A}_{0} \cup \mathbb{A}_{1}\right]$ since $\mathbb{A}_{0} \cup \mathbb{A}_{1}$ is locally countable, by Lemmas 3.1 and 4.1 .

\section{Background}

We now give some material to prepare the study of the Borel classes of rank two.

\section{$\underline{\text { Potential Wadge classes }}$}

In Theorem 1.3, $\mathbb{S}_{0} \cup \mathbb{S}_{1}$ is a subset of the body of a tree $T$ on $2^{2}$ which does not depend on $\boldsymbol{\Gamma}$. We first describe a simple version of $T$, which is sufficient to study the Borel classes (see [L6]). We identify $\left(2^{l}\right)^{2}$ and $\left(2^{2}\right)^{l}$, for each $l \in \omega+1$.

Definition 5.1 (1) Let $\mathcal{F} \subseteq \bigcup_{l \in \omega}\left(2^{l}\right)^{2} \equiv\left(2^{2}\right)^{<\omega}$. We say that $\mathcal{F}$ is a frame if

(a) $\forall l \in \omega \exists !\left(s_{l}, t_{l}\right) \in \mathcal{F} \cap\left(2^{l}\right)^{2}$,

(b) $\forall p, q \in \omega \forall w \in 2^{<\omega} \exists N \in \omega\left(s_{q} 0 w 0^{N}, t_{q} 1 w 0^{N}\right) \in \mathcal{F}$ and $\left(\left|s_{q} 0 w 0^{N}\right|-1\right)_{0}=p$,

(c) $\forall l>0 \exists q<l \exists w \in 2^{<\omega}\left(s_{l}, t_{l}\right)=\left(s_{q} 0 w, t_{q} 1 w\right)$.

(2) If $\mathcal{F}=\left\{\left(s_{l}, t_{l}\right) \mid l \in \omega\right\}$ is a frame, then we will call $T$ the tree on $2^{2}$ generated by $\mathcal{F}$ :

$$
T:=\left\{(s, t) \in\left(2^{2}\right)^{<\omega} \mid s=\emptyset \vee\left(\exists q \in \omega \exists w \in 2^{<\omega}(s, t)=\left(s_{q} 0 w, t_{q} 1 w\right)\right)\right\} .
$$

The existence condition in (a) and the density condition (b) ensure that $\lceil T\rceil$ is big enough to contain sets of arbitrary high potential complexity. The uniqueness condition in (a) and condition (c) ensure that $\lceil T\rceil$ is small enough to make the reduction in Theorem 1.3 possible. The last part of condition (b) gives a control on the verticals which is very useful to construct complicated examples. 
In the sequel, $T$ will be the tree generated by a fixed frame $\mathcal{F}$ (Lemma 3.3 in [L6] ensures the existence of concrete frames). Note that $\lceil T\rceil \subseteq N_{0} \times N_{1}$, which will be useful in the sequel (recall that $N_{s}$ is the basic clopen set of sequences beginning with $s \in 2^{<\omega}$ ).

\section{$\underline{\text { Acyclicity }}$}

We will use some material from [L6] and [L8], where some possibly different notions of acyclicity of the levels of $T$ are involved. We will check that they coincide in our case.

Definition 5.2 Let $X$ be a set, and $A$ be a relation on $X$.

(a) An $A$-path is a finite sequence $\left(x_{i}\right)_{i \leq n}$ of points of $X$ such that $\left(x_{i}, x_{i+1}\right) \in A$ if $i<n$.

(b) We say that $A$ is connected if for any $x, y \in X$ there is an $A$-path $\left(x_{i}\right)_{i \leq n}$ with $x_{0}=x$ and $x_{n}=y$.

(c) An $A$-cycle is an A-path $\left(x_{i}\right)_{i \leq L}$ with $L \geq 3,\left(x_{i}\right)_{i<L}$ is injective and $x_{L}=x_{0}$ (so that $A$ is acyclic if and only if there is no A-cycle).

Lemma 5.3 Let $l \in \omega$, and $T_{l}:=T \cap\left(2^{l}\right)^{2}$ be the lth level of $T$.

(a) $s\left(T_{l}\right)$ is connected and acyclic. In particular, $\lceil T\rceil$ is s-acyclic.

(b) A tree $S$ on $2^{2}$ has acyclic levels in the sense of [L6] if and only if $S$ has suitable levels in the sense of [L8], and this is the case of $T$.

Proof. (a) We argue by induction on $l$. The statement is clear for $l=0$. For the inductive step we use the fact that $T_{l+1}=\left\{(s \varepsilon, t \varepsilon) \mid(s, t) \in T_{l} \wedge \varepsilon \in 2\right\} \cup\left\{\left(s_{l} 0, t_{l} 1\right)\right\}$. As the map $s \varepsilon \mapsto s$ defines an isomorphism from $\left\{(s \varepsilon, t \varepsilon) \mid(s, t) \in T_{l}\right\}$ onto $T_{l}$, we are done. A cycle for $s(\lceil T\rceil)$ gives a cycle for $s\left(T_{l}\right)$, for $l$ big enough to ensure the injectivity of the initial segments.

(b) Assume that $S$ has acyclic levels in the sense of [L6]. This means that, for each $l$, the graph $G_{S_{l}}$ with set of vertices $2^{l} \oplus 2^{l}$ (with typical element $\overline{x_{\varepsilon}}:=\left(x_{\varepsilon}, \varepsilon\right) \in 2^{l} \times 2$ ) and set of edges

$$
\left\{\left\{\overline{x_{0}}, \overline{x_{1}}\right\} \mid \vec{x}:=\left(x_{0}, x_{1}\right) \in S_{l}\right\}
$$

is acyclic. We have to see that $S$ has suitable levels in the sense of [L8]. This means that, for each $l$, the following hold:

- $S_{l}$ is finite,

- $\exists \varepsilon \in 2 \quad x_{\varepsilon}^{0} \neq x_{\varepsilon}^{1}$ if $\overrightarrow{x^{0}} \neq \overrightarrow{x^{1}} \in S_{l}$,

- consider the graph $G^{S_{l}}$ with set of vertices $S_{l}$ and set of edges

$$
\left\{\left\{\overrightarrow{x^{0}}, \overrightarrow{x^{1}}\right\} \mid \overrightarrow{x^{0}} \neq \overrightarrow{x^{1}} \wedge \exists \varepsilon \in 2 \quad x_{\varepsilon}^{0}=x_{\varepsilon}^{1}\right\} ;
$$

then for any $G^{S_{l}}$-cycle $\left(\overrightarrow{x^{n}}\right)_{n \leq L}$, there are $\varepsilon \in 2$ and $k<m<n<L$ such that $x_{\varepsilon}^{k}=x_{\varepsilon}^{m}=x_{\varepsilon}^{n}$.

The first two properties are obvious. So assume that $\left(\overrightarrow{x^{n}}\right)_{n \leq L}$ is a $G^{S_{l}}$-cycle for which we cannot find $\varepsilon \in 2$ and $k<m<n<L$ such that $x_{\varepsilon}^{k}=x_{\varepsilon}^{m}=x_{\varepsilon}^{n}$. 
Case $1 x_{0}^{0}=x_{0}^{1}$.

Subcase 1.1 $L$ is odd.

Note that $L \geq 5$. Indeed, $L \geq 3$ since $\left(\overrightarrow{x^{n}}\right)_{n \leq L}$ is a $G^{S_{l}}$-cycle. So we just have to see that $L \neq 3$. As $x_{0}^{0}=x_{0}^{1}$ and $\overrightarrow{x^{0}} \neq \overrightarrow{x^{1}}, x_{1}^{0} \neq x_{1}^{1}$. By the choice of $\left(\overrightarrow{x^{n}}\right)_{n \leq L}, x_{0}^{1} \neq x_{0}^{2}$. Thus $x_{1}^{1}=x_{1}^{2}$. By the choice of $\left(\overrightarrow{x^{n}}\right)_{n \leq L}, x_{1}^{2} \neq x_{1}^{3}$. Thus $x_{0}^{2}=x_{0}^{3}$ and $x_{0}^{3} \neq x_{0}^{0}$. Therefore $\overrightarrow{x^{3}} \neq \overrightarrow{x^{0}}$ and $L \neq 3$.

Then $\overline{x_{0}^{0}}, \overline{x_{1}^{1}}, \overline{x_{0}^{2}}, \ldots, \overline{x_{1}^{L-2}}, \overline{x_{0}^{L-1}}$ is a $G_{S_{l}}$-cycle, by the choice of $\left(\overrightarrow{x^{n}}\right)_{n \leq L}$.

Subcase 1.2 $L$ is even, in which case $L \geq 4$.

Then $\overline{x_{0}^{0}}, \overline{x_{1}^{1}}, \overline{x_{0}^{2}}, \ldots, \overline{x_{1}^{L-1}}, \overline{x_{0}^{L}}$ is a $G_{S_{l}}$-cycle, by the choice of $\left(\overrightarrow{x^{n}}\right)_{n \leq L}$.

Case $2 x_{0}^{0} \neq x_{0}^{1}$.

The same arguments work, we just have to exchange the indexes.

- Conversely, assume that $\left(\overline{x_{\varepsilon_{n}}^{n}}\right)_{n \leq L}$ is a $G_{S_{l}}$-cycle. Then $L$ is even, and actually $L \geq 4$.

Case $1 \varepsilon_{0}=0$.

Then $\left(x_{\varepsilon_{0}}^{0}, x_{\varepsilon_{1}}^{1}\right),\left(x_{\varepsilon_{2}}^{2}, x_{\varepsilon_{1}}^{1}\right), \ldots,\left(x_{\varepsilon_{L-2}}^{L-2}, x_{\varepsilon_{L-1}}^{L-1}\right),\left(x_{\varepsilon_{L}}^{L}, x_{\varepsilon_{L-1}}^{L-1}\right),\left(x_{\varepsilon_{0}}^{0}, x_{\varepsilon_{1}}^{1}\right)$ is a $G^{S_{l} \text {-cycle of length }}$ $L+1$. If $\varepsilon \in 2$, then each $\varepsilon$ th coordinate appears exactly twice before the last element of the cycle.

Case $2 \varepsilon_{0}=1$.

The same argument works, we just have to exchange the coordinates.

- By Proposition 3.2 in [L6], $T$ has acyclic levels in the sense of [L6].

\section{The classes $\Pi_{2}^{0}$ and $\Sigma_{2}^{0}$}

\section{$\underline{\text { Example }}$}

We will use an example for $\boldsymbol{\Gamma}=\boldsymbol{\Pi}_{2}^{0}$ different from that in [L6], so that we prove the following.

Lemma 6.1 $\lceil T\rceil \cap \mathbb{E}_{0}$ is not separable from $\lceil T\rceil \backslash \mathbb{E}_{0}$ by a pot $\left(\Pi_{2}^{0}\right)$ set.

Proof. We argue by contradiction, which gives $P \in \operatorname{pot}\left(\boldsymbol{\Pi}_{2}^{0}\right)$, and also a dense $G_{\delta}$ subset $G$ of $2^{\omega}$ such that $P \cap G^{2} \in \Pi_{2}^{0}\left(G^{2}\right)$. Let $\left(O_{n}\right)_{n \in \omega}$ be a sequence of dense open subsets of $2^{\omega}$ with intersection $G$. Note that $\lceil T\rceil \cap \mathbb{E}_{0} \cap G^{2}=\lceil T\rceil \cap P \cap G^{2} \in \Delta_{2}^{0}\left(\lceil T\rceil \cap G^{2}\right)$. By Baire's theorem, it is enough to prove that $\lceil T\rceil \cap \mathbb{E}_{0} \cap G^{2}$ is dense and co-dense in the nonempty space $\lceil T\rceil \cap G^{2}$. So let $q \in \omega$ and $w \in 2^{<\omega}$. Pick $u_{0} \in 2^{\omega}$ such that $N_{s_{q} 0 w u_{0}} \subseteq O_{0}, v_{0} \in 2^{\omega}$ such that $N_{t_{q} 1 w u_{0} v_{0}} \subseteq O_{0}, u_{1} \in 2^{\omega}$ such that $N_{s_{q} 0 w u_{0} v_{0} u_{1}} \subseteq O_{1}, v_{1} \in 2^{\omega}$ such that $N_{t_{q} 1 w u_{0} v_{0} u_{1} v_{1}} \subseteq O_{1}$, and so on. 
Then $\left(s_{q} 0 w u_{0} v_{0} u_{1} v_{1} \ldots, t_{q} 1 w u_{0} v_{0} u_{1} v_{1} \ldots\right) \in\lceil T\rceil \cap \mathbb{E}_{0} \cap G^{2}$. Similarly, pick $N_{0} \in \omega$ such that $\left(s_{q} 0 w 0^{N_{0}}, t_{q} 1 w 0^{N_{0}}\right) \in \mathcal{F}, u_{0} \in 2^{\omega}$ such that $N_{s_{q} 0 w 0^{N_{0} 0 u_{0}}} \subseteq O_{0}, v_{0} \in 2^{\omega}$ such that $N_{t_{q} 1 w 0^{N_{0}} 1 u_{0} v_{0}} \subseteq O_{0}$, $N_{1} \in \omega$ such that $\left(s_{q} 0 w 0^{N_{0}} 0 u_{0} v_{0} 0^{N_{1}}, t_{q} 1 w 0^{N_{0}} 1 u_{0} v_{0} 0^{N_{1}}\right) \in \mathcal{F}, u_{1} \in 2^{\omega}$ such that

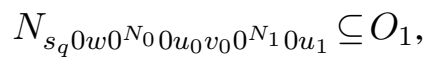

$v_{1} \in 2^{\omega}$ such that $N_{t_{q} 1 w 0^{N_{0}} 1 u_{0} v_{0} 0^{N_{1}} 1 u_{1} v_{1}} \subseteq O_{1}$, and so on. Then

$$
\left(s_{q} 0 w 0^{N_{0}} 0 u_{0} v_{0} 0^{N_{1}} 0 u_{1} v_{1} \ldots, t_{q} 1 w 0^{N_{0}} 1 u_{0} v_{0} 0^{N_{1}} 1 u_{1} v_{1} \ldots\right) \in\lceil T\rceil \cap G^{2} \backslash \mathbb{E}_{0} .
$$

This finishes the proof.

\section{The main result}

We reduce the study of disjoint analytic sets to that of disjoint Borel sets of low complexity, for the first classes we are considering.

Lemma 6.2 Let $X$ be a Polish space, and $A, B$ be disjoint analytic relations on $X$. Then exactly one of the following holds:

(a) the set $A$ is separable from $B$ by a pot $\left(\Pi_{2}^{0}\right)$ set,

(b) there is a $K_{\sigma}$ relation $A^{\prime} \subseteq A$ which is not pot $\left(\mathbf{\Pi}_{2}^{0}\right)$ such that $\overline{A^{\prime}} \backslash A^{\prime} \subseteq B$.

Proof. Theorem 1.10 in [L8] and Lemmas 6.1, 5.3 give $g, h: 2^{\omega} \rightarrow X$ continuous such that the inclusions $\lceil T\rceil \cap \mathbb{E}_{0} \subseteq(g \times h)^{-1}(A)$ and $\lceil T\rceil \backslash \mathbb{E}_{0} \subseteq(g \times h)^{-1}(B)$ hold. We set $A^{\prime}:=(g \times h)\left[\lceil T\rceil \cap \mathbb{E}_{0}\right]$, $B^{\prime}:=(g \times h)\left[\lceil T\rceil \backslash \mathbb{E}_{0}\right]$ and $C^{\prime}:=(g \times h)[\lceil T\rceil]$. Note that $A^{\prime}$ is a $K_{\sigma}$ subset of $A, B^{\prime} \subseteq B$, so that the compact set $C^{\prime}$ is the disjoint union of $A^{\prime}$ and $B^{\prime}$. As $\lceil T\rceil \cap \mathbb{E}_{0}$ is dense in $\lceil T\rceil, C^{\prime}$ is also the closure of $A^{\prime}$. As $\lceil T\rceil \cap \mathbb{E}_{0}=\lceil T\rceil \cap(g \times h)^{-1}\left(A^{\prime}\right), A^{\prime}$ is not $\operatorname{pot}\left(\boldsymbol{\Pi}_{2}^{0}\right)$, by Lemma 6.1.

Theorem 6.3 Let $X$ be a Polish space, and $A, B$ be disjoint analytic relations on $X$ such that $A$ is quasi-acyclic. Then one of the following holds:

(a) the set $A$ is separable from $B$ by a pot $\left(\Pi_{2}^{0}\right)$ set,

(b) there is $f: 2^{\omega} \rightarrow X$ injective continuous such that the inclusions $\lceil T\rceil \cap \mathbb{E}_{0} \subseteq(f \times f)^{-1}(A)$ and $\lceil T\rceil \backslash \mathbb{E}_{0} \subseteq(f \times f)^{-1}(B)$ hold.

Proof. Assume that (a) does not hold. By Lemma 6.2, we may assume that $B$ is the complement of $A$. Let $\left(C_{n}\right)_{n \in \omega}$ be a witness for the fact that $A$ is quasi-acyclic. Note that there are disjoint Borel subsets $O_{0}, O_{1}$ of $X$ such that $A \cap\left(O_{0} \times O_{1}\right)$ is not $\operatorname{pot}\left(\mathbf{\Pi}_{2}^{0}\right)$. We may assume that $X$ is zero-dimensional, the $C_{n}$ 's are closed, and $O_{0}, O_{1}$ are clopen, refining the topology if necessary. We can also replace $A$ and the $C_{n}$ 's with their intersection with $O_{0} \times O_{1}$ and assume that they are contained in $O_{0} \times O_{1}$.

- We may assume that $X$ is recursively presented, $O_{0}, O_{1} \in \Delta_{1}^{1}$ and the relation " $(x, y) \in C_{n}$ " is $\Delta_{1}^{1}$ in $(x, y, n)$. As $\Delta_{X}$ is Polish finer than the topology on $X, A \notin \Pi_{2}^{0}\left(X^{2}, \tau_{1}\right)$. We now perform the following derative on $A$. We set, for $F \in \Pi_{1}^{0}\left(X^{2}, \tau_{1}\right), F^{\prime}:=\overline{F \cap A}^{\tau_{1}} \cap \overline{F \backslash A}^{\tau_{1}}$ (see 22.30 in [K]). 
Then we inductively define, for any ordinal $\xi, F_{\xi}$ by

$$
\left\{\begin{array}{l}
F_{0}:=X^{2} \\
F_{\xi+1}:=F_{\xi}^{\prime} \\
F_{\lambda}:=\bigcap_{\xi<\lambda} F_{\xi} \text { if } \lambda \text { is limit }
\end{array}\right.
$$

(see 22.27 in $[\mathrm{K}])$. As $\left(F_{\xi}\right)$ is a decreasing sequence of closed subsets of the Polish space $\left(X^{2}, \tau_{1}\right)$, there is $\theta<\omega_{1}$ such that $F_{\theta}=F_{\theta+1}$. In particular, $F_{\theta}=F_{\theta+1}=F_{\theta}^{\prime}={\overline{F_{\theta}} \cap A}^{\tau_{1}} \cap{\overline{F_{\theta} \backslash A}}^{\tau_{1}}$, so that $F_{\theta} \cap A$ and $F_{\theta} \backslash A$ are $\tau_{1}$-dense in $F_{\theta}$.

- Let us prove that $F_{\theta}$ is not empty. We argue by contradiction:

$$
X^{2}=\neg F_{\theta}=\bigcup_{\xi \leq \theta} \neg F_{\xi}=\bigcup_{\xi \leq \theta}\left(\neg F_{\xi} \cap \bigcap_{\eta<\xi} F_{\eta}\right)=\bigcup_{\xi<\theta} F_{\xi} \backslash F_{\xi+1},
$$

so that $A=\bigcup_{\xi<\theta} A \cap F_{\xi} \backslash F_{\xi+1}$. But $A \cap F_{\xi} \backslash F_{\xi+1}=A \cap F_{\xi} \backslash\left(\bar{F}_{\xi} \cap A{ }^{\tau_{1}} \cap{\overline{F_{\xi} \backslash A}}^{\tau_{1}}\right)=F_{\xi} \backslash{\overline{F_{\xi} \backslash A}}^{\tau_{1}}$. This means that $\left(F_{\xi} \backslash F_{\xi+1}\right)_{\xi<\theta}$ is a countable partition of $\left(X^{2}, \tau_{1}\right)$ into $\boldsymbol{\Delta}_{2}^{0}$ sets, and that $A$ is $\boldsymbol{\Delta}_{2}^{0}$ on each piece of the partition. This implies that $A$ is $\Delta_{2}^{0}\left(X^{2}, \tau_{1}\right)$, which is absurd.

- Let us prove that $F_{\theta}$ is $\Sigma_{1}^{1}$. We use $7 \mathrm{C}$ in [Mo]. We define a set relation by

$$
\varphi(x, y, P) \Leftrightarrow(x, y) \notin(\neg P)^{\prime} .
$$

Note that $\varphi$ is monotone, and thus operative. It is also $\Pi_{1}^{1}$ on $\Pi_{1}^{1}$. By 3E.2, 3F.6 and 4B.2 in [Mo], we can apply 7C.8 in [Mo], so that $\varphi^{\infty}(x, y)$ is $\Pi_{1}^{1}$. An induction shows that $\varphi^{\xi}(x, y)$ is equivalent to " $(x, y) \notin F_{\xi+1}$ ". Thus $(x, y) \notin F_{\theta}$ is equivalent to $(x, y) \notin \bigcap_{\xi} F_{\xi}=\bigcap_{\xi} F_{\xi+1},(x, y) \in \bigcup_{\xi} \neg F_{\xi+1}$ and $\varphi^{\infty}(x, y)$.

- We are ready to prove the following key property:

$$
\forall q \in \omega \forall U, V \in \Sigma_{1}^{1}(X) \quad F_{\theta} \cap(U \times V) \neq \emptyset \Rightarrow \exists n \geq q \quad F_{\theta} \cap C_{n} \cap(U \times V) \neq \emptyset .
$$

Indeed, this property says that $I:=F_{\theta} \cap\left(\bigcup_{n \geq q} C_{n}\right)$ is $\Sigma_{X}^{2}$-dense in $F_{\theta}$ for each $q \in \omega$. We fix $q \in \omega$, and prove first that $I$ is $\tau_{1}$-dense in $F_{\theta}$. So let $U, V \in \Delta_{1}^{1}$ such that $F_{\theta} \cap(U \times V)$ is nonempty. As $F_{\theta} \backslash A$ is $\tau_{1}$-dense in $F_{\theta}$, we get $(x, y) \in\left(F_{\theta} \backslash A\right) \cap(U \times V)$. As $F_{\theta} \cap A$ is $\tau_{1}$-dense in $F_{\theta}$, we get $\left(x_{k}, y_{k}\right) \in F_{\theta} \cap A$ converving to $(x, y)$ for $\tau_{1}$. Pick $n_{k} \in \omega$ such that $\left(x_{k}, y_{k}\right) \in C_{n_{k}}$. As $C_{n_{k}}$ is closed, and thus $\tau_{1}$-closed, we may assume that the sequence $\left(n_{k}\right)_{k \in \omega}$ is strictly increasing. Now $\left(x_{k}, y_{k}\right) \in I \cap(U \times V)$ if $k$ is big enough. In order to get the statement for $\Sigma_{X}^{2}$, we have to note that $I$ is $\Sigma_{1}^{1}$ since $F_{\theta}$ is $\Sigma_{1}^{1}$ and the relation " $(x, y) \in C_{n}$ " is $\Delta_{1}^{1}$ in $(x, y, n)$. This implies that $\bar{I}^{\tau_{1}}=\bar{I}^{\Sigma_{X}^{2}}$, by a double application of the separation theorem. Therefore $F_{\theta} \subseteq \bar{I}^{\tau_{1}}=\bar{I}^{\Sigma_{X}^{2}}$ and $I$ is $\Sigma_{X}^{2}$-dense in $F_{\theta}$.

- We set, for $\vec{u}=\left(u_{0}, u_{1}\right) \in T \backslash\{\vec{\emptyset}\}$,

$$
\begin{aligned}
& n(\vec{u}):=\operatorname{Card}\left(\left\{i<|\vec{u}| \mid u_{0}(i) \neq u_{1}(i)\right\}\right), \\
& \vec{t}(\vec{u}):=\left(s_{q} 0, t_{q} 1\right) \text { if } \vec{u}=\left(s_{q} 0 w, t_{q} 1 w\right) .
\end{aligned}
$$


- We are ready for the construction of $f$. We construct the following objects:

- sequences $\left(x_{s}\right)_{s \in 2^{<\omega} \backslash\{\emptyset\}, s(0)=0},\left(y_{s}\right)_{s \in 2^{<\omega} \backslash\{\emptyset\}, s(0)=1}$ of points of $X$,

- sequences $\left(X_{s}\right)_{s \in 2<\omega} \backslash\{\emptyset\}, s(0)=0,\left(Y_{s}\right)_{s \in 2}<\omega \backslash\{\emptyset\}, s(0)=1$ of $\Sigma_{1}^{1}$ subsets of $X$,

- a map $\Phi:\{\vec{t}(\vec{u}) \mid \vec{u} \in T \backslash\{\vec{\emptyset}\}\} \rightarrow \omega$.

We want these objects to satisfy the following conditions:

(1) $x_{s} \in X_{s} \wedge y_{s} \in Y_{s}$

(2) $X_{s \varepsilon} \subseteq X_{s} \subseteq \Omega_{X} \cap O_{0} \wedge Y_{s \varepsilon} \subseteq Y_{s} \subseteq \Omega_{X} \cap O_{1}$

(3) $\operatorname{diam}_{\mathrm{GH}}\left(X_{s}\right), \operatorname{diam}_{\mathrm{GH}}\left(Y_{s}\right) \leq 2^{-|s|}$

(4) $\left(x_{u_{0}}, y_{u_{1}}\right) \in F_{\theta} \cap C_{\Phi(\vec{t}(\vec{u}))}$

(5) $\left(X_{u_{0}} \times Y_{u_{1}}\right) \cap\left(\bigcup_{n<n(\vec{u})} C_{n}\right)=\emptyset$

(6) $X_{s 0} \cap X_{s 1}=Y_{s 0} \cap Y_{s 1}=\emptyset$

- Assume that this has been done. As in the proof of Theorem 3.3, we get $f: N_{\varepsilon} \rightarrow O_{\varepsilon}$ injective continuous, so that $f: 2^{\omega} \rightarrow X$ is injective continuous. If $(\alpha, \beta) \in\lceil T\rceil \cap \mathbb{E}_{0}$, then $\Phi(\vec{t}((\alpha, \beta) \mid n))=N$ if $n$ is big enough. In this case, by (4), $\left(x_{\alpha \mid n}, y_{\beta \mid n}\right) \in C_{N}$ which is closed, so that $(f(\alpha), g(\beta)) \in C_{N} \subseteq A$. If $(\alpha, \beta) \in\lceil T\rceil \backslash \mathbb{E}_{0}$, then the sequence $(n((\alpha, \beta) \mid n))_{n>0}$ tends to infinity. Thus $(f(\alpha), g(\beta))$ is not in $\bigcup_{n \in \omega} C_{n}=A$ by (5).

- So let us prove that the construction is possible. The key property gives $\Phi(0,1) \geq 1$ and $\left(x_{0}, y_{1}\right)$ in $F_{\theta} \cap C_{\Phi(0,1)} \cap \Omega_{X^{2}}$. As $\Omega_{X^{2}} \subseteq \Omega_{X}^{2}, x_{0}, y_{1} \in \Omega_{X}$. We choose $\Sigma_{1}^{1}$ subsets $X_{0}, Y_{1}$ of $X$ with GHdiameter at most $2^{-1}$ such that $\left(x_{0}, y_{1}\right) \in X_{0} \times Y_{1} \subseteq\left(\left(\Omega_{X} \cap O_{0}\right) \times\left(\Omega_{X} \cap O_{1}\right)\right) \backslash C_{0}$, which completes the construction for the length $l=1$.

Let $l \geq 1$. We now want to build $x_{s}, X_{s}, y_{s}, Y_{s}$ for $s \in 2^{l+1}$, as well as $\Phi\left(s_{l} 0, t_{l} 1\right)$. Note that $\left(x_{s_{l}}, y_{t_{l}}\right) \in F_{\theta} \cap(U \times V)$, where

$$
\begin{array}{r}
U:=\left\{x_{s_{l}}^{\prime} \in X_{s_{l}} \mid \exists\left(x_{s}^{\prime}\right)_{s \in 2^{l} \backslash\left\{s_{l}\right\}, s(0)=0} \in \prod_{s \in 2^{l} \backslash\left\{s_{l}\right\}, s(0)=0} X_{s} \exists\left(y_{s}^{\prime}\right)_{s \in 2^{l}, s(0)=1} \in \prod_{s \in 2^{l}, s(0)=1} Y_{s}\right. \\
\left.\forall \vec{u} \in T \cap\left(2^{l} \times 2^{l}\right)\left(x_{u_{0}}^{\prime}, y_{u_{1}}^{\prime}\right) \in F_{\theta} \cap C_{\Phi(\vec{t}(\vec{u}))}\right\}, \\
V:=\left\{y_{t_{l}}^{\prime} \in Y_{t_{l}} \mid \exists\left(x_{s}^{\prime}\right)_{s \in 2^{l}, s(0)=0} \in \prod_{s \in 2^{l}, s(0)=0} X_{s} \exists\left(y_{s}^{\prime}\right)_{s \in 2^{l} \backslash\left\{t_{l}\right\}, s(0)=1} \in \prod_{s \in 2^{l} \backslash\left\{t_{l}\right\}, s(0)=1} Y_{s}\right. \\
\left.\forall \vec{u} \in T \cap\left(2^{l} \times 2^{l}\right)\left(x_{u_{0}}^{\prime}, y_{u_{1}}^{\prime}\right) \in F_{\theta} \cap C_{\Phi(\vec{t}(\vec{u}))}\right\} .
\end{array}
$$

The key property gives $\Phi\left(s_{l} 0, t_{l} 1\right)>\max \left(n\left(s_{l} 0, t_{l} 1\right), \max _{q<l} \Phi\left(s_{q} 0, t_{q} 1\right)\right)$ and

$$
\left(x_{s_{l} 0}, y_{t_{l} 1}\right) \in F_{\theta} \cap C_{\Phi\left(s_{l} 0, t_{l} 1\right)} \cap(U \times V) .
$$

The fact that $x_{s_{l} 0} \in U$ gives witnesses $\left(x_{s 0}\right)_{s \in 2^{l} \backslash\left\{s_{l}\right\}, s(0)=0}$ and $\left(y_{s 0}\right)_{s \in 2^{l}, s(0)=1}$. Similarly, the fact that $y_{t_{l} 1} \in V$ gives $\left(x_{s 1}\right)_{s \in 2^{l}, s(0)=0}$ and $\left(y_{s 1}\right)_{s \in 2^{l} \backslash\left\{t_{l}\right\}, s(0)=1}$. Note that $x_{s_{l} 0} \neq x_{s_{l} 1}$ because

$$
\left(x_{s_{l} 0}, y_{t_{l} 1}\right) \in C_{\Phi\left(s_{l} 0, t_{l} 1\right)},
$$

$\left(x_{s_{l} 1}, y_{t_{l} 1}\right) \in C_{\Phi\left(\vec{t}\left(s_{l} 1, t_{l} 1\right)\right)}$, and $\Phi\left(s_{l} 0, t_{l} 1\right)>\Phi\left(\vec{t}\left(s_{l} 1, t_{l} 1\right)\right)$. Similarly, $y_{t_{l} 0} \neq y_{t_{l} 1}$. If $s \in 2^{l}$, then the connectedness of $s\left(T_{l}\right)$ gives an injective $s(T)$-path $p_{s}$ from $s$ to $s_{l}$. This gives a $s(A)$-path from $x_{s 0}$ to $x_{s 1}$ if $s(0)=0$, and a $s(A)$-path from $y_{s 0}$ to $y_{s 1}$ if $s(0)=1$. Using the quasi-acyclicity of $A$, we see, by induction on the length of $p_{s}$, that $x_{s 0} \neq x_{s 1}$ and $y_{s 0} \neq y_{s 1}$. 
The following picture illustrates the situation when $l=2$.

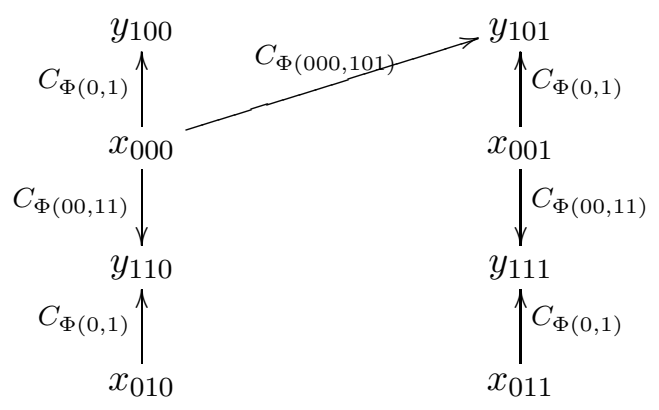

Then we take small enough $\Sigma_{1}^{1}$ neighborhoods of the $x_{s \varepsilon}$ 's and $y_{s \varepsilon}$ 's to complete the construction.

\section{Consequences}

Corollary 6.4 Let $X$ be a Polish space, and $A, B$ be disjoint analytic relations on $X$ such that $A$ is either s-acyclic, or locally countable. Then exactly one of the following holds:

(a) the set $A$ is separable from $B$ by a pot $\left(\Pi_{2}^{0}\right)$ set,

(b) there is $f: 2^{\omega} \rightarrow X$ injective continuous such that the inclusions $\lceil T\rceil \cap \mathbb{E}_{0} \subseteq(f \times f)^{-1}(A)$ and $\lceil T\rceil \backslash \mathbb{E}_{0} \subseteq(f \times f)^{-1}(B)$ hold.

Proof. By Lemma 6.1, $\lceil T\rceil \cap \mathbb{E}_{0}$ is not separable from $\lceil T\rceil \backslash \mathbb{E}_{0}$ by a $\operatorname{pot}\left(\boldsymbol{\Pi}_{2}^{0}\right)$ set. This shows that (a) and (b) cannot hold simultaneously. So assume that (a) does not hold. By Lemma 6.2, we may assume that $A$ is $\Sigma_{2}^{0}$ and $B$ is the complement of $A$. By Lemma 2.2. we may also assume that $A$ is quasi-acyclic. It remains to apply Theorem 6.3.

Corollary 6.5 Let $X, Y$ be Polish spaces, and $A, B$ be disjoint analytic subsets of $X \times Y$ such that $A$ is locally countable. Then exactly one of the following holds:

(a) the set $A$ is separable from $B$ by a pot $\left(\Pi_{2}^{0}\right)$ set,

(b) $\left(2^{\omega}, 2^{\omega},\lceil T\rceil \cap \mathbb{E}_{0},\lceil T\rceil \backslash \mathbb{E}_{0}\right) \sqsubseteq(X, Y, A, B)$.

Proof. As in the proof of Corollary 6.4, (a) and (b) cannot hold simultaneously. So assume that (a) does not hold. We argue as in the proof of Corollary 3.7. Corollary 6.4 gives $f^{\prime}: 2^{\omega} \rightarrow Z$.

Corollary 6.6 Let $X$ be a Polish space, and A, B be disjoint analytic relations on $X$. The following are equivalent:

(1) there is an s-acyclic relation $R \in \Sigma_{1}^{1}$ such that $A \cap R$ is not separable from $B \cap R$ by a pot $\left(\mathbf{\Pi}_{2}^{0}\right)$ set,

(2) there is $f: 2^{\omega} \rightarrow X$ injective continuous with $\lceil T\rceil \cap \mathbb{E}_{0} \subseteq(f \times f)^{-1}(A)$ and $\lceil T\rceil \backslash \mathbb{E}_{0} \subseteq(f \times f)^{-1}(B)$.

Proof. (1) $\Rightarrow$ (2) We apply Corollary 6.4

$(2) \Rightarrow(1)$ We can take $R:=(f \times f)[\lceil T\rceil]$.

Remark. There is a version of Corollary 6.6 for $\Sigma_{2}^{0}$ instead of $\Pi_{2}^{0}$, obtained by exchanging the roles of $A$ and $B$. This symmetry is not present in Theorem 6.3 , 
Corollary 6.7 Let $X$ be a Polish space, and $A, B$ be disjoint analytic relations on $X$ such that $A$ is contained in a $\operatorname{pot}\left(F_{\sigma}\right)$ s-acyclic relation, or $A \cup B$ is s-acyclic. Then exactly one of the following holds:

(a) the set $A$ is separable from $B$ by a pot $\left(\Sigma_{2}^{0}\right)$ set,

(b) there is $f: 2^{\omega} \rightarrow X$ injective continuous such that the inclusions $\lceil T\rceil \backslash \mathbb{E}_{0} \subseteq(f \times f)^{-1}(A)$ and $\lceil T\rceil \cap \mathbb{E}_{0} \subseteq(f \times f)^{-1}(B)$ hold.

Proof. Let $R$ be a $\operatorname{pot}\left(F_{\sigma}\right)$ s-acyclic relation containing $A$. Then there is no $\operatorname{pot}\left(\Sigma_{2}^{0}\right)$ set $P$ separating $A \cap R=A$ from $B \cap R$, since otherwise $P \cap R \in \operatorname{pot}\left(\boldsymbol{\Sigma}_{2}^{0}\right)$ and separates $A$ from $B$. Corollary 6.6 gives $f: 2^{\omega} \rightarrow X$ injective continuous with $\lceil T\rceil \cap \mathbb{E}_{0} \subseteq(f \times f)^{-1}(B)$ and $\lceil T\rceil \backslash \mathbb{E}_{0} \subseteq(f \times f)^{-1}(A)$.

If $A \cup B$ is s-acyclic, then we apply Corollary 6.4.

Remarks. (1) Corollary 6.7 also holds when $A \cup B$ is locally countable, but we did not mention it in the statement since (a) always holds in this case. Indeed, by reflection, $A \cup B$ is contained in a locally countable Borel set $C$. As $A, B$ are disjoint analytic sets, there is a Borel set $D$ separating $A$ from $B$. Thus $C \cap D$ is a locally countable Borel set separating $A$ from $B$. But a locally countable Borel set has $\Sigma_{2}^{0}$ vertical sections, and is therefore $\operatorname{pot}\left(\Sigma_{2}^{0}\right)$ (see [Lo2]).

(2) There is a version of Corollary 6.7 for $\boldsymbol{\Gamma}=\Sigma_{1}^{0}$, where we replace the class $F_{\sigma}$ with the class of open sets. We do not state it since (a) always holds in this case. Indeed, a potentially open s-acyclic relation is a countable union of Borel rectangles for which at least one side is a singleton, so that this union is potentially clopen, just like any of its Borel subsets.

\section{The class $\Delta_{2}^{0}$}

\section{Example}

We set, for each $\varepsilon \in 2$,

$$
\mathbb{E}_{0}^{\varepsilon}:=\left\{(\alpha, \beta) \in 2^{\omega} \times 2^{\omega} \mid \exists m>0 \quad \alpha(m) \neq \beta(m) \wedge \forall n>m \alpha(n)=\beta(n) \wedge(m-1)_{0} \equiv \varepsilon(\bmod 2)\right\} .
$$

Lemma 7.1 $\lceil T\rceil \cap \mathbb{E}_{0}^{0}$ is not separable from $\lceil T\rceil \cap \mathbb{E}_{0}^{1}$ by a pot $\left(\boldsymbol{\Delta}_{2}^{0}\right)$ set.

Proof. The proof is similar to that of Lemma 6.1. We argue by contradiction, which gives $D$ in $\operatorname{pot}\left(\boldsymbol{\Delta}_{2}^{0}\right)$, and also a dense $G_{\delta}$ subset $G$ of $2^{\omega}$ such that $D \cap G^{2} \in \boldsymbol{\Delta}_{2}^{0}\left(G^{2}\right)$. Let $\left(O_{n}\right)_{n \in \omega}$ be a sequence of dense open subsets of $2^{\omega}$ with intersection $G$. Note that $\lceil T\rceil \cap \mathbb{E}_{0}^{0} \cap G^{2} \subseteq\lceil T\rceil \cap D \cap G^{2}$, $\lceil T\rceil \cap \mathbb{E}_{0}^{1} \cap G^{2} \subseteq\lceil T\rceil \cap G^{2} \backslash D$ and $\lceil T\rceil \cap D \cap G^{2} \in \Delta_{2}^{0}\left(\lceil T\rceil \cap G^{2}\right)$. By Baire's theorem, it is enough to prove that $\lceil T\rceil \cap \mathbb{E}_{0}^{0} \cap G^{2}$ and $\lceil T\rceil \cap \mathbb{E}_{0}^{1} \cap G^{2}$ are dense in $\lceil T\rceil \cap G^{2}$. Let us do it for $\lceil T\rceil \cap \mathbb{E}_{0}^{0} \cap G^{2}$, the other case being similar. So let $q \in \omega$ and $w \in 2^{<\omega}$. Pick $N \in \omega$ such that $\left(s_{q} 0 w 0^{N_{0}}, t_{q} 1 w 0^{N}\right)$ is in $\mathcal{F}$ and $\left(\left|s_{q} 0 w 0^{N}\right|-1\right)_{0}=0$. Then we argue as in the proof of of Lemma 6.1; pick $u_{0} \in 2^{\omega}$ with $N_{s_{q} 0 w 0^{N} 0 u_{0}} \subseteq O_{0}, v_{0} \in 2^{\omega}$ with $N_{t_{q} 1 w 0^{N} 1 u_{0} v_{0}} \subseteq O_{0}, u_{1} \in 2^{\omega}$ with $N_{s_{q} 0 w 0^{N} 0 u_{0} v_{0} u_{1}} \subseteq O_{1}, v_{1} \in 2^{\omega}$ with $N_{t_{q} 1 w 0^{N} 1 u_{0} v_{0} u_{1} v_{1}} \subseteq O_{1}$, and so on. Then $\left(s_{q} 0 w 0^{N} 0 u_{0} v_{0} u_{1} v_{1} \ldots, t_{q} 1 w 0^{N} 1 u_{0} v_{0} u_{1} v_{1} \ldots\right)$ is in $\lceil T\rceil \cap \mathbb{E}_{0}^{0} \cap G^{2}$. 


\section{The main result}

We will prove a version of Theorem 6.3 for the class $\Delta_{2}^{0}$.

Theorem 7.2 Let $X$ be a Polish space, and $A, B$ be disjoint analytic relations on $X$ such that $A \cup B$ is quasi-acyclic. Then one of the following holds:

(a) the set $A$ is separable from $B$ by a pot $\left(\Delta_{2}^{0}\right)$ set,

(b) there is $f: 2^{\omega} \rightarrow X$ injective continuous such that the inclusions $\lceil T\rceil \cap \mathbb{E}_{0}^{0} \subseteq(f \times f)^{-1}(A)$ and $\lceil T\rceil \cap \mathbb{E}_{0}^{1} \subseteq(f \times f)^{-1}(B)$ hold.

Proof. The proof is similar to that of of Theorem 6.3. Assume that (a) does not hold. By Lemma 3.5. we may assume that $A, B$ are $\Sigma_{2}^{0}$. Let $\left(C_{n}\right)_{n \in \omega}$ be a witness for the fact that $A \cup B$ is quasiacyclic. As $A, B$ are $\boldsymbol{\Sigma}_{2}^{0}$, we may assume that each $C_{n}$ is either contained in $A$, or contained in $B$. Note that there are disjoint Borel subsets $O_{0}, O_{1}$ of $X$ such that $A \cap\left(O_{0} \times O_{1}\right)$ is not separable from $B \cap\left(O_{0} \times O_{1}\right)$ by a $\operatorname{pot}\left(\boldsymbol{\Delta}_{2}^{0}\right)$ set. We may assume that $X$ is zero-dimensional, the $C_{n}$ 's are closed, and $O_{0}, O_{1}$ are clopen, refining the topology if necessary. We can also replace $A, B$ and the $C_{n}$ 's with their intersection with $O_{0} \times O_{1}$ and assume that they are contained in $O_{0} \times O_{1}$. This gives a sequence $\left(C_{n}^{0}\right)_{n \in \omega}$ (resp., $\left(C_{n}^{1}\right)_{n \in \omega}$ ) of pairwise disjoint closed relations on $X$ with union $A$ (resp., $B$ ).

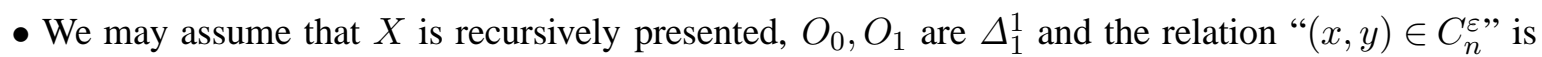
$\Delta_{1}^{1}$ in $(x, y, \varepsilon, n)$. As $\Delta_{X}$ is Polish finer than the topology on $X, A$ is not separable from $B$ by a $\boldsymbol{\Delta}_{2}^{0}\left(X^{2}, \tau_{1}\right)$ set. We set, for $F \in \Pi_{1}^{0}\left(X^{2}, \tau_{1}\right), F^{\prime}:=\overline{F \cap A}^{\tau_{1}} \cap{\overline{F \cap B^{\tau}}}^{\tau_{1}}$ (see 22.30 in $[\mathrm{K}]$ ). Then

$$
F_{\theta}=F_{\theta+1}=F_{\theta}^{\prime}={\overline{F_{\theta} \cap A}}^{\tau_{1}} \cap{\overline{F_{\theta} \cap B}}^{\tau_{1}},
$$

so that $F_{\theta} \cap A$ and $F_{\theta} \cap B$ are $\tau_{1}$-dense in $F_{\theta}$.

- Let us prove that $F_{\theta}$ is not empty. We argue by contradiction, so that $A=\bigcup_{\xi<\theta} A \cap F_{\xi} \backslash F_{\xi+1}$. But $A \cap F_{\xi} \backslash F_{\xi+1}=A \cap F_{\xi} \backslash\left({\overline{F_{\xi} \cap A^{\prime}}}^{\tau_{1}} \cap{\overline{F_{\xi} \cap B}}^{\tau_{1}}\right) \subseteq F_{\xi} \backslash{\overline{F_{\xi} \cap B}}^{\tau_{1}} \subseteq \neg B$. This means that $\left(F_{\xi} \backslash F_{\xi+1}\right)_{\xi<\theta}$ is a countable partition of $\left(X^{2}, \tau_{1}\right)$ into $\Delta_{2}^{0}$ sets, and that $A$ is separable from $B$ by a $\Delta_{2}^{0}$ set on each piece of the partition. This implies that $A$ is separable from $B$ by a $\Delta_{2}^{0}\left(X^{2}, \tau_{1}\right)$ set, which is absurd.

- As in the proof of Theorem 6.3, $F_{\theta}$ is $\Sigma_{1}^{1}$, and the following key property holds:

$$
\forall \varepsilon \in 2 \forall q \in \omega \quad \forall U, V \in \Sigma_{1}^{1}(X) \quad F_{\theta} \cap(U \times V) \neq \emptyset \Rightarrow \exists n \geq q \quad F_{\theta} \cap C_{n}^{\varepsilon} \cap(U \times V) \neq \emptyset .
$$

- We construct again sequences $\left(x_{s}\right),\left(y_{s}\right),\left(X_{s}\right),\left(Y_{s}\right)$ and $\Phi$ satisfying the following conditions:

(1) $x_{s} \in X_{s} \wedge y_{s} \in Y_{s}$

(2) $X_{s \varepsilon} \subseteq X_{s} \subseteq \Omega_{X} \cap O_{0} \wedge Y_{s \varepsilon} \subseteq Y_{s} \subseteq \Omega_{X} \cap O_{1}$

(3) $\operatorname{diam}_{\mathrm{GH}}\left(X_{s}\right), \operatorname{diam}_{\mathrm{GH}}\left(Y_{s}\right) \leq 2^{-|s|}$

(4) $\left(x_{u_{0}}, y_{u_{1}}\right) \in F_{\theta} \cap C_{\Phi(\vec{t}(\vec{u}))}^{\varepsilon}$ if $(|\vec{t}(\vec{u})|-2)_{0} \equiv \varepsilon(\bmod 2)$, with the convention $(-1)_{0}=0$

(5) $X_{s 0} \cap X_{s 1}=Y_{s 0} \cap Y_{s 1}=\emptyset$

- Assume that this has been done. If $(\alpha, \beta) \in\lceil T\rceil \cap \mathbb{E}_{0}^{0}$, then $\Phi(\vec{t}((\alpha, \beta) \mid n))=N$ if $n$ is big enough. In this case, by (4), $\left(x_{\alpha \mid n}, y_{\beta \mid n}\right) \in C_{N}^{0}$ which is closed, so that $(f(\alpha), g(\beta)) \in C_{N}^{0} \subseteq A$. Similarly, if $(\alpha, \beta) \in\lceil T\rceil \cap \mathbb{E}_{0}^{1}$, then $(f(\alpha), g(\beta)) \in C_{N}^{1} \subseteq B$. 
- So let us prove that the construction is possible. The key property gives $\Phi(0,1) \in \omega$ and $\left(x_{0}, y_{1}\right)$ in $F_{\theta} \cap C_{\Phi(0,1)}^{0} \cap \Omega_{X^{2}}$. We choose $\Sigma_{1}^{1}$ subsets $X_{0}, Y_{1}$ of $X$ with GH-diameter at most $2^{-1}$ such that $\left(x_{0}, y_{1}\right) \in X_{0} \times Y_{1} \subseteq\left(\Omega_{X} \cap O_{0}\right) \times\left(\Omega_{X} \cap O_{1}\right)$, which completes the construction for the length $l=1$.

Let $l \geq 1$. We now want to build $x_{s}, X_{s}, y_{s}, Y_{s}$ for $s \in 2^{l+1}$, as well as $\Phi\left(s_{l} 0, t_{l} 1\right)$. Fix $\eta \in 2$ such that $(l-1)_{0} \equiv \eta(\bmod 2)$. Note that $\left(x_{s_{l}}, y_{t_{l}}\right) \in F_{\theta} \cap(U \times V)$, where

$$
\begin{array}{r}
U:=\left\{x_{s_{l}}^{\prime} \in X_{s_{l}} \mid \exists\left(x_{s}^{\prime}\right)_{s \in 2^{l} \backslash\left\{s_{l}\right\}, s(0)=0} \in \Pi_{s \in 2^{l} \backslash\left\{s_{l}\right\}, s(0)=0} X_{s} \exists\left(y_{s}^{\prime}\right)_{s \in 2^{l}, s(0)=1} \in \Pi_{s \in 2^{l}, s(0)=1} Y_{s}\right. \\
\left.\forall \vec{u} \in T \cap\left(2^{l} \times 2^{l}\right)\left(x_{u_{0}}^{\prime}, y_{u_{1}}^{\prime}\right) \in F_{\theta} \cap C_{\Phi(\vec{t}(\vec{u}))}^{\varepsilon} \text { if }(|\vec{t}(\vec{u})|-2)_{0} \equiv \varepsilon(\bmod 2)\right\}, \\
V:=\left\{y_{t_{l}}^{\prime} \in Y_{t_{l}} \mid \exists\left(x_{s}^{\prime}\right)_{s \in 2^{l}, s(0)=0} \in \Pi_{s \in 2^{l}, s(0)=0} X_{s} \exists\left(y_{s}^{\prime}\right)_{s \in 2^{l} \backslash\left\{t_{l}\right\}, s(0)=1} \in \Pi_{s \in 2^{l} \backslash\left\{t_{l}\right\}, s(0)=1} Y_{s}\right. \\
\left.\forall \vec{u} \in T \cap\left(2^{l} \times 2^{l}\right)\left(x_{u_{0}}^{\prime}, y_{u_{1}}^{\prime}\right) \in F_{\theta} \cap C_{\Phi(\vec{t}(\vec{u}))}^{\varepsilon} \text { if }(|\vec{t}(\vec{u})|-2)_{0} \equiv \varepsilon(\bmod 2)\right\} .
\end{array}
$$

The key property gives $\Phi\left(s_{l} 0, t_{l} 1\right)>\max _{q<l} \Phi\left(s_{q} 0, t_{q} 1\right)$ and

$$
\left(x_{s_{l} 0}, y_{t_{l} 1}\right) \in F_{\theta} \cap C_{\Phi\left(s_{l} 0, t_{l} 1\right)}^{\eta} \cap(U \times V) .
$$

Note that $x_{s_{l} 0} \neq x_{s_{l} 1}$ because $\left(x_{s_{l} 0}, y_{t_{l} 1}\right) \in C_{\Phi\left(s_{l} 0, t_{l} 1\right)}^{\eta},\left(x_{s_{l} 1}, y_{t_{l} 1}\right) \in C_{\Phi\left(\vec{t}\left(s_{l} 1, t_{l} 1\right)\right)}^{\varepsilon}$ if

$$
\left(\left|\vec{t}\left(s_{l} 1, t_{l} 1\right)\right|-2\right)_{0} \equiv \varepsilon(\bmod 2),
$$

and $\Phi\left(s_{l} 0, t_{l} 1\right)>\Phi\left(\vec{t}\left(s_{l} 1, t_{l} 1\right)\right)$. Similarly, $y_{t_{l} 0} \neq y_{t_{l} 1}$. If $s \in 2^{l}$, then there is an injective $s(T)$-path $p_{s}$ from $s$ to $s_{l}$. This gives a $s(A \cup B)$-path from $x_{s 0}$ to $x_{s 1}$ if $s(0)=0$, and a $s(A \cup B)$-path from $y_{s 0}$ to $y_{s 1}$ if $s(0)=1$. Using the quasi-acyclicity of $s(A \cup B)$, we see, by induction on the length of $p_{s}$, that $x_{s 0} \neq x_{s 1}$ and $y_{s 0} \neq y_{s 1}$.

\section{Consequences}

Corollary 7.3 Let $X$ be a Polish space, and $A, B$ be disjoint analytic relations on $X$ such that

- either $A \cup B$ is either s-acyclic or locally countable

- or $A$ is contained in a pot $\left(\boldsymbol{\Delta}_{2}^{0}\right)$ s-acyclic or locally countable relation.

Then exactly one of the following holds:

(a) the set $A$ is separable from $B$ by a pot $\left(\Delta_{2}^{0}\right)$ set,

(b) there is $f: 2^{\omega} \rightarrow X$ injective continuous such that the inclusions $\lceil T\rceil \cap \mathbb{E}_{0}^{0} \subseteq(f \times f)^{-1}(A)$ and $\lceil T\rceil \cap \mathbb{E}_{0}^{1} \subseteq(f \times f)^{-1}(B)$ hold.

Proof. By Lemma 7.1, $\lceil T\rceil \cap \mathbb{E}_{0}^{0}$ is not separable from $\lceil T\rceil \cap \mathbb{E}_{0}^{1}$ by a $\operatorname{pot}\left(\boldsymbol{\Delta}_{2}^{0}\right)$ set. This shows that (a) and (b) cannot hold simultaneously. So assume that (a) does not hold.

- If $A \cup B$ is s-acyclic or locally countable, then by Lemma 3.5, we may assume that $A, B$ are $\Sigma_{2}^{0}$. By Lemma 2.2. we may also assume that $A \cup B$ is quasi-acyclic. It remains to apply Theorem 7.2 .

- Assume that $R$ is $\operatorname{pot}\left(\boldsymbol{\Delta}_{2}^{0}\right)$ and contains $A$. Then there is no $\operatorname{pot}\left(\boldsymbol{\Delta}_{2}^{0}\right)$ set $P$ separating $A \cap R=A$ from $B \cap R$, since otherwise $P \cap R \in \operatorname{pot}\left(\Delta_{2}^{0}\right)$ separates $A$ from $B$. It remains to apply the first point. This finishes the proof. 
Corollary 7.4 Let $X, Y$ be Polish spaces, and $A, B$ be disjoint analytic subsets of $X \times Y$ such that $A \cup B$ is locally countable or $A$ is contained in a pot $\left(\Delta_{2}^{0}\right)$ locally countable set. Then exactly one of the following holds:

(a) the set $A$ is separable from $B$ by a pot $\left(\Delta_{2}^{0}\right)$ set,

(b) $\left(2^{\omega}, 2^{\omega},\lceil T\rceil \cap \mathbb{E}_{0}^{0},\lceil T\rceil \cap \mathbb{E}_{0}^{1}\right) \sqsubseteq(X, Y, A, B)$.

Proof. As in the proof of Corollary 7.3, (a) and (b) cannot hold simultaneously. Then we argue as in the proof of Corollary 3.7. The set $A^{\prime} \cup B^{\prime}$ is locally countable or $A^{\prime}$ is contained in a $\operatorname{pot}\left(\boldsymbol{\Delta}_{2}^{0}\right)$ locally countable set, and $A^{\prime}$ is not separable from $B^{\prime}$ by a $\operatorname{pot}\left(\Delta_{2}^{0}\right)$ set. Corollary 7.3 gives $f^{\prime}: 2^{\omega} \rightarrow Z$.

Corollary 7.5 Let $X$ be a Polish space, and A, B be disjoint analytic relations on $X$. The following are equivalent:

(1) there is an s-acyclic or locally countable relation $R \in \Sigma_{1}^{1}$ such that $A \cap R$ is not separable from $B \cap R$ by a $\operatorname{pot}\left(\boldsymbol{\Delta}_{2}^{0}\right)$ set,

(2) there is $f: 2^{\omega} \rightarrow X$ injective continuous with $\lceil T\rceil \cap \mathbb{E}_{0}^{0} \subseteq(f \times f)^{-1}(A)$ and $\lceil T\rceil \cap \mathbb{E}_{0}^{1} \subseteq(f \times f)^{-1}(B)$.

Proof. (1) $\Rightarrow(2)$ We apply Corollary 7.3

(2) $\Rightarrow(1)$ We can take $R:=(f \times f)\left[\lceil T\rceil \cap \mathbb{E}_{0}\right]$.

\section{The classes $D_{n}\left(\Sigma_{2}^{0}\right)$ and $\check{D}_{n}\left(\Sigma_{2}^{0}\right)$}

\section{Examples}

Notation. Let $\eta \geq 1$ be a countable ordinal, and $S_{\eta}: \omega \rightarrow \eta$ be onto. We set

$$
C_{0}:=\left\{\alpha \in 2^{\omega} \mid \exists m \in \omega \quad \forall p \geq m \quad \alpha(p)=0\right\}
$$

and, for $1 \leq \theta<\eta, C_{\theta}:=\left\{\alpha \in 2^{\omega} \mid \exists m \in \omega \forall p \in \omega \quad \alpha(<m, p>)=0 \wedge S_{\eta}\left((m)_{0}\right) \leq \theta\right\}$, so that $\left(C_{\theta}\right)_{\theta<\eta}$ is an increasing sequence of $\Sigma_{2}^{0}$ subsets of $2^{\omega}$. We then set $D_{\eta}:=D\left(\left(C_{\theta}\right)_{\theta<\eta}\right)$.

Lemma 8.1 The set $D_{\eta}$ is $D_{\eta}\left(\Sigma_{2}^{0}\right)$-complete.

Proof. By 21.14 in [K], it is enough to see that $D_{\eta}$ is not $\check{D}_{\eta}\left(\boldsymbol{\Sigma}_{2}^{0}\right)$ since it is $D_{\eta}\left(\boldsymbol{\Sigma}_{2}^{0}\right)$. We will prove more. Let us say that a pair $(\theta, F)$ is suitable if $\theta \leq \eta, F$ is a chain of finite binary sequences, $I_{F}:=\bigcap_{s \in F}\left\{\alpha \in N_{s} \mid(\alpha)_{|s|}=0^{\infty}\right\}$ is not empty and $S_{\eta}\left((|s|)_{0}\right) \geq \theta$ for each $s \in F$. Let us prove that $I_{F} \cap D\left(\left(C_{\theta^{\prime}}\right)_{\theta^{\prime}<\theta}\right)$ is not $\check{D}_{\theta}\left(\Sigma_{2}^{0}\right)$ if $(\theta, F)$ is suitable. This will give the result since $(\eta, \emptyset)$ is suitable.

We argue by induction on $\theta$. If $\theta=1$, then the $\Sigma_{2}^{0}$ set $I_{F} \cap C_{0}$ is dense and co-dense in the closed set $I_{F}$, so that it is not $\Pi_{2}^{0}$, by Baire's theorem. Assume the result proved for $\theta^{\prime}<\theta$. We argue by contradiction, which gives an increasing sequence $\left(H_{\theta^{\prime}}\right)_{\theta^{\prime}<\theta}$ of $\Sigma_{2}^{0}$ sets with

$$
I_{F} \cap D\left(\left(C_{\theta^{\prime}}\right)_{\theta^{\prime}<\theta}\right)=\neg D\left(\left(H_{\theta^{\prime}}\right)_{\theta^{\prime}<\theta}\right) .
$$


As $\neg\left(\bigcup_{\theta^{\prime}<\theta} C_{\theta^{\prime}}\right)$ is comeager in $I_{F}, I_{F} \cap \bigcup_{\theta^{\prime}<\theta} H_{\theta^{\prime}}$ too, which gives $\theta^{\prime}<\theta$ with parity opposite to that of $\theta$ and $s^{\prime} \supseteq \max _{s \in F} s$ such that $S_{\eta}\left(\left(\left|s^{\prime}\right|\right)_{0}\right)=\theta^{\prime}$ and $\emptyset \neq I_{F} \cap N_{s^{\prime}} \subseteq H_{\theta^{\prime}}$. We set $F^{\prime}:=F \cup\left\{s^{\prime}\right\}$, so that $\left(\theta^{\prime}, F^{\prime}\right)$ is suitable. By induction assumption, $I_{F^{\prime}} \cap D\left(\left(C_{\theta^{\prime \prime}}\right)_{\theta^{\prime \prime}<\theta^{\prime}}\right)$ is not $\check{D}_{\theta^{\prime}}\left(\Sigma_{2}^{0}\right)$. But $I_{F^{\prime}} \cap D\left(\left(C_{\theta^{\prime \prime}}\right)_{\theta^{\prime \prime}<\theta^{\prime}}\right)=I_{F^{\prime}} \backslash D\left(\left(H_{\theta^{\prime \prime}}\right)_{\theta^{\prime \prime}<\theta^{\prime}}\right) \in \check{D}_{\theta^{\prime}}\left(\Sigma_{2}^{0}\right)$ since $I_{F^{\prime}} \subseteq C_{\theta^{\prime}}$, which is absurd.

Notation. We now fix an effective frame in the sense of Definition 2.1 in [L8], which are frames in the sense of Definition 5.1 Lemma 2.3 in [L8] proves the existence of such an effective frame. Note that $\left(s_{1}, t_{1}\right)=(0,1)$, so that $s_{1}(0) \neq t_{1}(0)$. But $s_{l+1}(l)=t_{l+1}(l)$ if $l \geq 1$. Indeed, it is enough to see that $\left(\left((l)_{1}\right)_{1}\right)_{0}+\left(\left((l)_{1}\right)_{1}\right)_{1}<l$ in this case, by the proof of Lemma 2.3 in [L8]. As $(q)_{0}+(q)_{1} \leq q$, and $(q)_{0}+(q)_{1}<q$ if $q \geq 2$, we may assume that $\left((l)_{1}\right)_{1} \in 2$. If $\left((l)_{1}\right)_{1}=0$, then we are done since $l \geq 1$. If $\left((l)_{1}\right)_{1}=1$, then $l \geq 2$ and we are done too.

- The shift map $\mathcal{S}: 2^{L} \rightarrow 2^{L-1}$ is defined by $\mathcal{S}(\alpha)(m):=\alpha(m+1)$ when $1 \leq L \leq \omega$, with the convention $\omega-1:=\omega$.

- The symmetric difference $\alpha \Delta \beta$ of $\alpha, \beta \in 2^{L}$ is the element of $2^{L}$ defined by $(\alpha \Delta \beta)(m)=1$ exactly when $\alpha(m) \neq \beta(m)$, if $L \leq \omega$.

- We set $\mathbb{N}_{\eta}:=\left\{(\alpha, \beta) \in\lceil T\rceil \mid \mathcal{S}(\alpha \Delta \beta) \notin D_{\eta}\right\}$.

Lemma 8.2 The $\check{D}_{\eta}\left(\boldsymbol{\Sigma}_{2}^{0}\right)$ set $\mathbb{N}_{\eta}$ is not separable from $\lceil T\rceil \backslash \mathbb{N}_{\eta}$ by a pot $\left(D_{\eta}\left(\boldsymbol{\Sigma}_{2}^{0}\right)\right)$ set.

Proof. As $\lceil T\rceil$ is closed, $D_{\eta}$ is $D_{\eta}\left(\boldsymbol{\Sigma}_{2}^{0}\right)$ and $\mathcal{S}, \Delta$ are continuous, $\mathbb{N}_{\eta}$ is $\check{D}_{\eta}\left(\boldsymbol{\Sigma}_{2}^{0}\right)$. By Lemma 2.6 in [L8], it is enough to check that $D_{\eta}$ is ccs (see Definition 2.5 in [L8]). We just have to check that the $C_{\theta}$ 's are ccs. So let $\alpha, \alpha_{0} \in 2^{\omega}$ and $F: 2^{\omega} \rightarrow 2^{\omega}$ satisfying the conclusion of Lemma 2.4.(b) in [L8]. Note that $\alpha \in C_{0}$ exactly when $\{m \in \omega \mid \alpha(m)=1\}$ is finite, so that $C_{0}$ is ccs. If $\theta \geq 1$, then $\alpha \notin C_{\theta}$ exactly when, for each $m, S_{\eta}\left((m)_{0}\right) \leq \theta$ or there is $p$ with $\alpha(<m, p>)=1$. As $\left(B_{\alpha}(<m, p>)\right)_{0}=(<m, p>)_{0}=m, C_{\theta}$ is ccs too.

\section{The main result}

Notation. From now on, $\eta<\omega$. We set, for $2 \leq \theta \leq \eta$ and $(s, t) \in(2 \times 2)^{<\omega} \backslash\{(\emptyset, \emptyset)\}$,

$$
m_{s, t}^{\theta}:=\min \left\{m \in \omega \mid(\mathcal{S}(s \Delta t))_{m} \subseteq 0^{\infty} \wedge S_{\eta}\left((m)_{0}\right)<\theta\right\} .
$$

We also set $s^{-}:=<s(0), \ldots, s(|s|-2)>$ if $s \in 2^{<\omega}$. 
- We define the following relation on $(2 \times 2)^{<\omega}$ :

$$
\begin{array}{r}
(s, t) R\left(s^{\prime}, t^{\prime}\right) \Leftrightarrow(s, t) \subseteq\left(s^{\prime}, t^{\prime}\right) \wedge\left(|s| \leq 1 \vee\left(|s| \geq 2 \wedge \exists 2 \leq \theta \leq \eta m_{s, t}^{\theta} \neq m_{s^{-}, t^{-}}^{\theta} \wedge\right.\right. \\
\left.\forall(s, t) \subseteq\left(s^{\prime \prime}, t^{\prime \prime}\right) \subseteq\left(s^{\prime}, t^{\prime}\right) \forall \theta<\theta^{\prime} \leq \eta m_{s, t}^{\theta^{\prime}}=m_{s^{-}, t^{-}}^{\theta^{\prime}}=m_{s^{\prime \prime}, t^{\prime \prime}}^{\theta^{\prime}}\right) \vee \\
\quad(|s| \geq 2 \wedge s(|s|-1) \neq t(|s|-1) \wedge \\
\left.\forall(s, t) \subseteq\left(s^{\prime \prime}, t^{\prime \prime}\right) \subseteq\left(s^{\prime}, t^{\prime}\right) \forall 2 \leq \theta \leq \eta m_{s, t}^{\theta}=m_{s^{-}, t^{-}}^{\theta}=m_{s^{\prime \prime}, t^{\prime \prime}}^{\theta}\right) \vee \\
\left(|s| \geq 2 \wedge \forall(s, t) \subseteq\left(s^{\prime \prime}, t^{\prime \prime}\right) \subseteq\left(s^{\prime}, t^{\prime}\right) \quad\left(\forall 2 \leq \theta \leq \eta m_{s, t}^{\theta}=m_{s^{-}, t^{-}}^{\theta}=m_{s^{\prime \prime}, t^{\prime \prime}}^{\theta}\right) \wedge\right. \\
\left.\left.s^{\prime \prime}\left(\left|s^{\prime \prime}\right|-1\right)=t^{\prime \prime}\left(\left|s^{\prime \prime}\right|-1\right)\right)\right) .
\end{array}
$$

Note that $R$ is a tree relation, which means that it is a partial order (it contains the diagonal, is antisymmetric and transitive) with minimum element $(\emptyset, \emptyset)$, the set of predecessors of any sequence is finite and lineary ordered by $R$. Moreover, $R$ is distinguished in $\subseteq$, which means that $(s, t) R\left(s^{\prime}, t^{\prime}\right)$ if $(s, t) \subseteq\left(s^{\prime}, t^{\prime}\right) \subseteq\left(s^{\prime \prime}, t^{\prime \prime}\right)$ and $(s, t) R\left(s^{\prime \prime}, t^{\prime \prime}\right)$ (see [D-SR]).

- We set

$$
\begin{aligned}
& D_{\eta}:=\left\{(s, t) \in T|| s \mid \geq 2 \Rightarrow m_{s, t}^{\eta} \neq m_{s^{-}, t^{-}}^{\eta}\right\} \text { if } \eta \geq 2, \\
& D_{\theta}:=\left\{(s, t) \in T|| s \mid \geq 2 \wedge m_{s, t}^{\theta} \neq m_{s^{-}, t^{-}}^{\theta} \wedge \forall \theta<\theta^{\prime} \leq \eta m_{s, t}^{\theta^{\prime}}=m_{s^{-}, t^{-}}^{\theta^{\prime}}\right\} \text { if } 2 \leq \theta<\eta, \\
& D_{1}:=\left\{(s, t) \in T|| s \mid \geq 2 \wedge \forall 2 \leq \theta \leq \eta m_{s, t}^{\theta}=m_{s^{-}, t^{-}}^{\theta} \wedge s(|s|-1) \neq t(|s|-1)\right\}, \\
& D_{0}:=\{(s, t) \in T|| s \mid \geq 2 \wedge s(|s|-1)=t(|s|-1)\},
\end{aligned}
$$

so that the $\left(D_{\theta}\right)_{\theta \leq \eta}$ is a partition of $T$.

Theorem 8.3 Let $1 \leq \eta<\omega$. Let $X$ be a Polish space, and $A_{0}, A_{1}$ be disjoint analytic relations on $X$ such that $A_{0} \cup A_{1}$ is s-acyclic. Then exactly one of the following holds:

(a) the set $A_{0}$ is separable from $A_{1}$ by a pot $\left(D_{\eta}\left(\Sigma_{2}^{0}\right)\right)$ set,

(b) $\left(2^{\omega}, 2^{\omega}, \mathbb{N}_{\eta},\lceil T\rceil \backslash \mathbb{N}_{\eta}\right) \sqsubseteq\left(X, X, A_{0}, A_{1}\right)$, via a square map.

Proof. By Lemma 8.2, (a) and (b) cannot hold simultaneously. So assume that (a) does not hold. Note first that we may assume that $A_{0} \cup A_{1}$ is compact and $A_{1}$ is $D_{\eta}\left(\boldsymbol{\Sigma}_{2}^{0}\right)$. Indeed, Theorems 1.9 and 1.10 in [L8] give $\mathbb{S} \in D_{\eta}\left(\boldsymbol{\Sigma}_{2}^{0}\right)(\lceil T\rceil)$ and $f^{\prime}, g^{\prime}: 2^{\omega} \rightarrow X$ continuous such that the inclusions $\mathbb{S} \subseteq\left(f^{\prime} \times g^{\prime}\right)^{-1}\left(A_{1}\right)$ and $\lceil T\rceil \backslash \mathbb{S} \subseteq\left(f^{\prime} \times g^{\prime}\right)^{-1}\left(A_{0}\right)$ hold. Let $\left(\Sigma_{\theta}\right)_{\theta<\eta}$ be an increasing sequence of $\Sigma_{2}^{0}(\lceil T\rceil)$ sets with $\mathbb{S}=D\left(\left(\Sigma_{\theta}\right)_{\theta<\eta}\right), K:=\left(f^{\prime} \times g^{\prime}\right)[\lceil T\rceil]$, and $R_{\theta}:=\left(f^{\prime} \times g^{\prime}\right)\left[\Sigma_{\theta}\right]$. Note that $K$ is compact, $R_{\theta}$ is $K_{\sigma}$, $D\left(\left(R_{\theta}\right)_{\theta<\eta}\right) \subseteq A_{1}, K \backslash D\left(\left(R_{\theta}\right)_{\theta<\eta}\right) \subseteq A_{0}, D\left(\left(R_{\theta}\right)_{\theta<\eta}\right)=K \cap A_{1}, K \backslash D\left(\left(R_{\theta}\right)_{\theta<\eta}\right)=K \cap A_{0}$, so that $D\left(\left(R_{\theta}\right)_{\theta<\eta}\right)$ is not separable from $K \backslash D\left(\left(R_{\theta}\right)_{\theta<\eta}\right)$ by a $\operatorname{pot}\left(\check{D}_{\eta}\left(\Sigma_{2}^{0}\right)\right)$ set. So we can replace $A_{1}, A_{0}$ with $D\left(\left(R_{\theta}\right)_{\theta<\eta}\right), K \backslash D\left(\left(R_{\theta}\right)_{\theta<\eta}\right)$, respectively. 
- We may also assume that $X$ is zero-dimensional and there are disjoint clopen subsets $O_{0}, O_{1}$ of $X$ such that $A_{0} \cap\left(O_{0} \times O_{1}\right)$ is not separable from $A_{1} \cap\left(O_{0} \times O_{1}\right)$ by a $\operatorname{pot}\left(D_{\eta}\left(\boldsymbol{\Sigma}_{2}^{0}\right)\right)$ set. So, without loss of generality, we will assume that $A_{0} \cup A_{1} \subseteq O_{0} \times O_{1}$. We may also assume that $X$ is recursively presented, $A_{0}, A_{1}, O_{0}, O_{1}, R_{\theta}$ are $\Delta_{1}^{1}$, and $R_{\theta}$ is the union of $\Delta_{1}^{1} \cap \Pi_{1}^{0} \subseteq \Sigma_{1}^{1} \cap \Pi_{1}^{0}\left(\tau_{1}\right) \subseteq \Sigma_{1}^{0}\left(\tau_{2}\right)$ sets.

We set, for $\theta<\eta, N_{\theta}:=R_{\theta} \backslash\left(\bigcup_{\theta^{\prime}<\theta} R_{\theta^{\prime}}\right) \cap \bigcap_{\theta^{\prime}<\theta}{\overline{N_{\theta^{\prime}}}}^{\tau_{2}}$. Note that the $N_{\theta}$ 's are pairwise disjoint, which will be useful in the construction to get the injectivity of our reduction maps. We use the notation of Theorem 3.2 For simplicity, we set $F_{\theta}^{\varepsilon}:=F_{\theta, 2}^{\varepsilon}$.

Claim. (a) Assume that $k+1<\eta$. Then $F_{k}^{\varepsilon}={\overline{N_{k}}}^{\tau_{2}} \cup E_{k}$, where $E_{k} \subseteq \neg R_{k+1}$ is $\tau_{2}$-closed.

(b) $A_{0} \cap \bigcap_{\theta<\eta} F_{\theta}^{\varepsilon}=N_{\eta}:=K \backslash\left(\bigcup_{\theta<\eta} R_{\theta}\right) \cap \bigcap_{\theta<\eta}{\overline{N_{\theta}}}^{\tau_{2}}$.

(a) Indeed, we argue by induction on $k$ to prove (a). In the proof of this claim, all the closures will refer to $\tau_{2}$. Note first that $R_{0} \subseteq A_{\varepsilon} \subseteq R_{0} \cup \neg R_{1}$, so that $F_{0}^{\varepsilon}=\overline{A_{\varepsilon}}=\overline{R_{0}} \cup E_{0}=\overline{N_{0}} \cup E_{0}$. Then, inductively,

$$
\begin{aligned}
F_{k+1}^{\varepsilon} & =\overline{A_{1-|\operatorname{parity}(k)-\varepsilon|} \cap F_{k}^{\varepsilon}}=\overline{A_{1-|\operatorname{parity}(k)-\varepsilon|} \cap\left(\overline{N_{k}} \cup E_{k}\right)} \\
& =\overline{\left(\left(R_{k+1} \backslash R_{k}\right) \cup\left(R_{k+3} \backslash R_{k+2}\right) \ldots\right) \cap\left(\overline{N_{k}} \cup E_{k}\right)}=\overline{N_{k+1}} \cup E_{k+1} .
\end{aligned}
$$

(b) Note then that $F_{\eta-1}^{\varepsilon}=\overline{A_{1} \cap \bigcap_{k+1<\eta} F_{k}^{\varepsilon}}=\overline{A_{1} \cap \bigcap_{k+1<\eta}\left(\overline{N_{k}} \cup E_{k}\right)}=\overline{N_{\eta-1}}$, so that

$$
A_{0} \cap \bigcap_{\theta<\eta} F_{\theta}^{\varepsilon}=K \backslash\left(\bigcup_{\theta<\eta} R_{\theta}\right) \cap \bigcap_{\theta<\eta} \overline{N_{\theta}}
$$

This proves the claim.

- We construct the following objects:

- sequences $\left(x_{s}\right)_{s \in 2<\omega, 0 \subseteq s},\left(y_{s}\right)_{s \in 2<\omega, 1 \subseteq s}$ of points of $X$,

- sequences $\left(X_{s}\right)_{s \in 2<\omega, 0 \subseteq s},\left(Y_{s}\right)_{s \in 2<\omega, 1 \subseteq s}$ of $\Sigma_{1}^{1}$ subsets of $X$,

- a sequence $\left(U_{s, t}\right)_{(s, t) \in T \backslash\{(\emptyset, \emptyset)\}}$ of $\Sigma_{1}^{1}$ subsets of $X^{2}$.

We want these objects to satisfy the following conditions:

(1) $x_{s} \in X_{s} \wedge y_{s} \in Y_{s} \wedge\left(x_{s}, y_{t}\right) \in U_{s, t}$

(2) $X_{s \varepsilon} \subseteq X_{s} \subseteq \Omega_{X} \cap O_{0} \wedge Y_{s \varepsilon} \subseteq Y_{s} \subseteq \Omega_{X} \cap O_{1} \wedge U_{s, t} \subseteq \Omega_{X^{2}} \cap\left(X_{s} \times Y_{t}\right)$

(3) $\operatorname{diam}_{\mathrm{GH}}\left(X_{s}\right), \operatorname{diam}_{\mathrm{GH}}\left(Y_{s}\right), \operatorname{diam}_{\mathrm{GH}}\left(U_{s, t}\right) \leq 2^{-|s|}$

(4) $X_{s 0} \cap X_{s 1}=Y_{s 0} \cap Y_{s 1}=\emptyset$

(5) $\left((s, t) R\left(s^{\prime}, t^{\prime}\right) \wedge \exists \theta \leq 2(s, t),\left(s^{\prime}, t^{\prime}\right) \in D_{\theta}\right) \Rightarrow U_{s^{\prime}, t^{\prime}} \subseteq U_{s, t}$

(6) $U_{s, t} \subseteq N_{\theta}$ if $(s, t) \in D_{\theta}$

(7) $(s, t) R\left(s^{\prime}, t^{\prime}\right) \Rightarrow U_{s^{\prime}, t^{\prime}} \subseteq{\overline{U_{s, t}}}^{\tau}$ 
- Assume that this has been done. As in the proof of Theorem 3.3 we get $f: 2^{\omega} \rightarrow X$ injective continuous. If $(\alpha, \beta) \in \mathbb{N}_{\eta}$, then we can find $\theta<\eta$ of parity opposite to that of $\eta$ and $\left(n_{k}\right)_{k \in \omega}$ strictly increasing such that $(\alpha, \beta) \mid n_{k} \in D_{\theta}$ and $(\alpha, \beta)\left|n_{k} R(\alpha, \beta)\right| n_{k+1}$ for each $k \in \omega$. In this case, by (1)-(3) and (5)-(6), $\left(U_{(\alpha, \beta) \mid n_{k}}\right)_{k \in \omega}$ is a decreasing sequence of nonempty clopen subsets of $A_{0} \cap \Omega_{X^{2}}$ with vanishing diameters, so that its intersection is a singleton $\{F(\alpha, \beta)\} \subseteq A_{0}$. As $\left(x_{\alpha \mid n}, y_{\beta \mid n}\right)$ converges (for $\Sigma_{X^{2}}$ and thus for $\Sigma_{X}^{2}$ ) to $F(\alpha, \beta),(f(\alpha), f(\beta))=F(\alpha, \beta) \in A_{0}$. If $(\alpha, \beta) \in\lceil T\rceil \backslash \mathbb{N}_{\eta}$, then we argue similarly to see that $(f(\alpha), f(\beta)) \in A_{1}$.

- So let us prove that the construction is possible. Let $\left(x_{0}, y_{1}\right) \in N_{\eta} \cap \Omega_{X^{2}}, X_{0}, Y_{1}$ be $\Sigma_{1}^{1}$ subsets of $X$ with diameter at most $2^{-1}$ such that $x_{0} \in X_{0} \subseteq \Omega_{X} \cap O_{0}$ and $y_{1} \in Y_{1} \subseteq \Omega_{X} \cap O_{1}$, and $U_{0,1}$ be a $\Sigma_{1}^{1}$ subset of $X^{2}$ with diameter at most $2^{-1}$ such that $\left(x_{0}, y_{1}\right) \in U_{0,1} \subseteq N_{\eta} \cap \Omega_{X^{2}} \cap\left(X_{0} \times Y_{1}\right)$. This completes the construction for $l=1$ since $(0,1) \in D_{\eta}$.

- Note that $\left(0^{2}, 1^{2}\right) \in D_{\eta}$ since $m_{0,1}^{\eta}=0$ and $m_{0^{2}, 1^{2}}^{\eta}=1$ if $\eta \geq 2$. We set $S_{0}:={\overline{U_{0,1}}}^{\tau_{1}} \cap\left(X_{0} \times Y_{1}\right)$ and $S_{1}:=S_{0} \cap N_{0} \cap \Omega_{X^{2}}$. As $U_{0,1} \subseteq{\overline{N_{0}}}^{\tau_{2}}, S_{0} \subseteq{\overline{S_{1}}}^{\tau_{1}}$. In particular, $\Pi_{\varepsilon}\left[S_{1}\right]$ is $\Sigma_{X}$-dense in $\Pi_{\varepsilon}\left[S_{0}\right]$ for each $\varepsilon \in 2$, by continuity of the projections. As $\left(x_{0}, y_{1}\right) \in U_{0,1} \cap\left(\Pi_{0}\left[S_{0}\right] \times \Pi_{1}\left[S_{0}\right]\right)$, this implies that $U_{0,1} \cap\left(\Pi_{0}\left[S_{1}\right] \times \Pi_{1}\left[S_{1}\right]\right)$ is not empty and contains some $\left(x_{0^{2}}, y_{1^{2}}\right)$ (the projections maps are open). This gives $y_{10} \in X$ with $\left(x_{0^{2}}, y_{10}\right) \in S_{1}$, and $x_{01} \in X$ with $\left(x_{01}, y_{1^{2}}\right) \in S_{1}$. As $U_{0,1} \subseteq N_{\eta}$ and $S_{1} \subseteq N_{0}$, $x_{0^{2}} \neq x_{01}$ and $y_{10} \neq y_{1^{2}}$. It remains to choose $\Sigma_{1}^{1}$ subsets $X_{0^{2}}, X_{01}, Y_{10}, Y_{1^{2}}$ of $X$ with diameter at most $2^{-2}$ such that $\left(x_{0 \varepsilon}, y_{1 \varepsilon}\right) \in X_{0 \varepsilon} \times Y_{1 \varepsilon} \subseteq X_{0} \times Y_{1}$ and $X_{0^{2}} \cap X_{01}=Y_{10} \cap Y_{1^{2}}=\emptyset$, as well as $\Sigma_{1}^{1}$ subsets $U_{0^{2}, 1^{2}}, U_{0^{2}, 10}, U_{01,1^{2}}$ of $X^{2}$ with diameter at most $2^{-2}$ such that $\left(x_{0^{2}}, y_{1^{2}}\right) \in U_{0^{2}, 1^{2}} \subseteq U_{0,1} \cap\left(X_{0^{2}} \times Y_{1^{2}}\right)$ and $\left(x_{0 \varepsilon}, y_{1 \varepsilon}\right) \in U_{0 \varepsilon, 1 \varepsilon} \subseteq{\overline{U_{0,1}}}^{\tau_{1}} \cap N_{0} \cap \Omega_{X^{2}} \cap\left(X_{0 \varepsilon} \times Y_{1 \varepsilon}\right)$. This completes the construction for $l=2$.

- Assume that our objects are constructed for the level $l \geq 2$, which is the case for $l=2$. Note that $\left(s_{l} 0, t_{l} 1\right) \notin D_{0}$, and we already noticed that $s_{l}(l-1)=t_{l}(l-1)$ since $l \geq 2$, so that $\left(s_{l}, t_{l}\right) \in D_{0}$. We set $(\tilde{s}, \tilde{t}):=\left(s_{l-1} 0, t_{l-1} 1\right)$ (which is not in $\left.D_{0}\right)$, and

$$
\begin{aligned}
& S_{0}:=\left\{\left(\left(\bar{x}_{s}\right)_{s \in 2^{l}, 0 \subseteq s},\left(\bar{y}_{t}\right)_{t \in 2^{l}, 1 \subseteq t}\right) \in X^{2^{l}} \mid \forall(s, t) \in T \cap\left(2^{l} \times 2^{l}\right) \backslash\{(\tilde{s}, \tilde{t})\} \quad\left(\bar{x}_{s}, \bar{y}_{t}\right) \in U_{s, t} \wedge\right. \\
& \left.\left(\bar{x}_{\tilde{s}}, \bar{y}_{\tilde{t}}\right) \in{\overline{N_{0}}}^{\tau_{2}} \cap{\overline{U_{\tilde{s}, \tilde{t}}}}^{\tau_{1}} \cap\left(X_{\tilde{s}} \times Y_{\tilde{t}}\right)\right\},
\end{aligned}
$$

$S_{1}:=\left\{\left(\left(\bar{x}_{s}\right)_{s \in 2^{l}, 0 \subseteq s},\left(\bar{y}_{t}\right)_{t \in 2^{l}, 1 \subseteq t}\right) \in S_{0} \mid\left(\bar{x}_{\tilde{s}}, \bar{y}_{\tilde{t}}\right) \in N_{0} \cap \Omega_{X^{2}}\right\}$.

We equip $X^{2^{l}}$ with the product of the Gandy-Harrington topologies. Let us show that $S_{1}$ is dense in $S_{0}$. Let $\left(\mathcal{U}_{s}\right)_{s \in 2^{l}, 0 \subseteq s}$ and $\left(\mathcal{V}_{t}\right)_{t \in 2^{l}, 1 \subseteq t}$ be sequences of $\Sigma_{1}^{1}$ sets with

$$
\left(\left(\Pi_{s \in 2^{l}, 0 \subseteq s} \mathcal{U}_{s}\right) \times\left(\Pi_{t \in 2^{l}, 1 \subseteq t} \mathcal{V}_{t}\right)\right) \cap S_{0} \neq \emptyset
$$

with witness $\left(\left(x_{s}^{\prime}\right),\left(y_{t}^{\prime}\right)\right), \mathcal{A}_{\varepsilon}:=\left\{s \in 2^{l} \mid s(l-1)=\varepsilon\right\}$, and

$$
\begin{array}{r}
U:=\left\{\bar{x}_{\tilde{s}} \in \mathcal{U}_{\tilde{s}} \mid \exists\left(\bar{x}_{s}\right)_{s \in \mathcal{A}_{0} \backslash\{\tilde{s}\}} \in \Pi_{s \in \mathcal{A}_{0} \backslash\{\tilde{s}\}} \mathcal{U}_{s} \exists\left(\bar{y}_{t}\right)_{t \in \mathcal{A}_{0}} \in \prod_{t \in \mathcal{A}_{0}} \mathcal{V}_{t}\right. \\
\left.\forall(s, t) \in T \cap\left(\mathcal{A}_{0} \times \mathcal{A}_{0}\right) \quad\left(\bar{x}_{s}, \bar{y}_{t}\right) \in U_{s, t}\right\}, \\
V:=\left\{\bar{y}_{\tilde{t}} \in \mathcal{V}_{\tilde{t}} \mid \exists\left(\bar{x}_{s}\right)_{s \in \mathcal{A}_{1}} \in \Pi_{s \in \mathcal{A}_{1}} \mathcal{U}_{s} \exists\left(\bar{y}_{t}\right)_{t \in \mathcal{A}_{1} \backslash\{\tilde{t}\}} \in \Pi_{t \in \mathcal{A}_{1} \backslash\{\tilde{t}\}} \mathcal{V}_{t}\right. \\
\left.\forall(s, t) \in T \cap\left(\mathcal{A}_{1} \times \mathcal{A}_{1}\right)\left(\bar{x}_{s}, \bar{y}_{t}\right) \in U_{s, t}\right\} .
\end{array}
$$


Then $\left(x_{\tilde{s}}^{\prime}, y_{\tilde{t}}^{\prime}\right) \in{\overline{N_{0}}}^{\tau_{2}} \cap{\overline{U_{\tilde{s}, \tilde{t}}}}^{\tau_{1}} \cap(U \times V)$. This gives $\left(\bar{x}_{\tilde{s}}, \bar{y}_{\tilde{t}}\right)$ in $N_{0} \cap{\overline{U_{\tilde{s}, \tilde{t}}}}^{\tau_{1}} \cap(U \times V) \cap \Omega_{X^{2}}$. We choose witnesses $\left(\bar{x}_{s}\right)_{s \in \mathcal{A}_{0} \backslash\{\tilde{s}\}},\left(\bar{y}_{t}\right)_{t \in \mathcal{A}_{0}}$ (resp., $\left.\left(\bar{x}_{s}\right)_{s \in \mathcal{A}_{1}},\left(\bar{y}_{t}\right)_{t \in \mathcal{A}_{1} \backslash\{\tilde{t}\}}\right)$ for the fact that $\bar{x}_{\tilde{s}} \in U$ (resp., $\left.\bar{y}_{\tilde{t}} \in V\right)$. Then $\left(\left(\bar{x}_{s}\right),\left(\bar{y}_{t}\right)\right) \in\left(\left(\Pi_{s \in 2^{l}, 0 \subseteq s} \mathcal{U}_{t}\right) \times\left(\Pi_{t \in 2^{l}, 1 \subseteq t} \mathcal{V}_{t}\right)\right) \cap S_{1}$, as desired.

The sets $U_{\varepsilon}:=\Pi_{s_{l}}\left[S_{\varepsilon}\right]$ and $V_{\varepsilon}:=\Pi_{t_{l}}\left[S_{\varepsilon}\right]$ are $\Sigma_{1}^{1}$ sets. As $S_{1}$ is dense in $S_{0}, U_{1}$ (resp., $V_{1}$ ) is dense in $U_{0}$ (resp., $V_{0}$ ). Note that $\left(x_{s_{l}}, y_{t_{l}}\right) \in U_{s_{l}, t_{l}} \cap\left(U_{0} \times V_{0}\right)$. As $U_{1}$ (resp,. $V_{1}$ ) is dense in $U_{0}$ (resp., $\left.V_{0}\right), U_{s_{l}, t_{l}}$ meets $U_{1} \times V_{1}$.

Let $\left(s_{l} 0, t_{l} 1\right)^{R}$ be the $R$-predecessor of $\left(s_{l} 0, t_{l} 1\right)$. Assume first that $\left(s_{l} 0, t_{l} 1\right) \in D_{\eta}$. Then $\left(s_{l} 0, t_{l} 1\right)^{R} \in D_{\eta}$ too. Note that $U_{s_{l}, t_{l}} \subseteq{\overline{U_{\left(s_{l} 0, t_{l} 1\right)^{R}}}}^{\tau_{1}}$ since $\left(s_{l} 0, t_{l} 1\right)^{R} R\left(s_{l}, t_{l}\right)$. Thus ${\bar{U}\left(s_{l} 0, t_{l} 1\right)^{R}}^{\tau_{1}}$ meets $U_{1} \times V_{1}$. This gives $\left(x_{s_{l} 0}, y_{t_{l} 1}\right) \in U_{\left(s_{l} 0, t_{l} 1\right)^{R}} \cap\left(U_{1} \times V_{1}\right)$. We choose witnesses $\left(x_{s 0}\right)_{s \in 2^{l} \backslash\left\{s_{l}\right\}, 0 \subseteq s}$, $\left(y_{t 0}\right)_{t \in 2^{l}, 1 \subseteq t}$ (resp., $\left(x_{s 1}\right)_{s \in 2^{l}, 0 \subseteq s},\left(y_{t 1}\right)_{t \in 2^{l} \backslash\left\{t_{l}\right\}, 1 \subseteq t}$ ) for the fact that $x_{s_{l} 0} \in U_{1}$ (resp., $y_{t_{l} 1} \in V_{1}$ ). As $\left(x_{s_{l} 0}, y_{t_{l} 1}\right) \in U_{\left(s_{l} 0, t_{l} 1\right)^{R}} \subseteq N_{\eta}$ and $\left(x_{s_{l} \varepsilon}, y_{t_{l} \varepsilon}\right) \in N_{0}, x_{s_{l} 0} \neq x_{s_{l} 1}$ and $y_{t_{l} 0} \neq y_{t_{l} 1}$. As in the proof of Theorem 3.3 , the s-acyclicity of $A_{0} \cup A_{1}$ and the fact that $O_{0}, O_{1}$ are disjoint ensure the fact that $x_{s 0} \neq x_{s 1}$ and $y_{t 0} \neq y_{t 1}$ for $s, t$ arbitrary with the right first coordinate. Then we choose $\Sigma_{1}^{1}$ subsets $X_{s \varepsilon}, Y_{t \varepsilon}$ of $X$ with diameter at most $2^{-l-1}$ such that $\left(x_{s \varepsilon}, y_{t \varepsilon}\right) \in X_{s \varepsilon} \times Y_{t \varepsilon} \subseteq X_{s} \times Y_{t}$ and $X_{s 0} \cap X_{s 1}=Y_{s 0} \cap Y_{s 1}=\emptyset$, as well as $\Sigma_{1}^{1}$ subsets $U_{s \varepsilon, t \varepsilon^{\prime}}$ of $X^{2}$, with diameter at most $2^{-l-1}$, containing $\left(x_{s \varepsilon}, y_{t \varepsilon^{\prime}}\right)$ and contained in $X_{s \varepsilon} \times Y_{t \varepsilon}$, such that

- $U_{s_{l} 0, t_{l} 1} \subseteq U_{\left(s_{l} 0, t_{l} 1\right)^{R}}$

- $U_{\tilde{s} \varepsilon, \tilde{t} \varepsilon} \subseteq \bar{U}_{\tilde{s}, \tilde{t}}^{\tau_{1}} \cap N_{0} \cap \Omega_{X^{2}}$,

- $U_{s \varepsilon, t \varepsilon} \subseteq U_{s, t}$ if $(s, t) \neq(\tilde{s}, \tilde{t})$.

The argument is the same if $\left(s_{l} 0, t_{l} 1\right),\left(s_{l} 0, t_{l} 1\right)^{R} \in D_{\theta}$. So it remains to study the case where $\left(s_{l} 0, t_{l} 1\right) \in D_{\theta^{\prime}}$ and $\left(s_{l} 0, t_{l} 1\right)^{R} \in D_{\theta}$, and $\theta^{\prime}<\theta$. In this case, note that $U_{\left(s_{l} 0, t_{l} 1\right)^{R}} \cap\left(U_{1} \times V_{1}\right)$ is not empty and contained in $N_{\theta} \subseteq{\overline{N_{\theta^{\prime}}}}^{\tau_{2}}$. This gives $\left(x_{s_{l} 0}, y_{t_{l} 1}\right) \in N_{\theta^{\prime}} \cap \overline{U_{\left(s_{l} 0, t_{l} 1\right)^{R}}{ }^{\tau}} \cap \Omega_{X^{2}} \cap\left(U_{1} \times V_{1}\right)$, and we conclude as before.

\section{Consequences}

Corollary 8.4 Let $1 \leq \eta<\omega, X$ be a Polish space, and $A, B$ be disjoint analytic relations on $X$ such that $A$ is contained in a pot $\left(\boldsymbol{\Delta}_{2}^{0}\right)$ s-acyclic relation. Then exactly one of the following holds:

(a) the set $A$ is separable from $B$ by a pot $\left(D_{\eta}\left(\Sigma_{2}^{0}\right)\right)$ set,

(b) $\left(2^{\omega}, 2^{\omega}, \mathbb{N}_{\eta},\lceil T\rceil \backslash \mathbb{N}_{\eta}\right) \sqsubseteq(X, X, A, B)$, via a square map.

Proof. Let $R$ be a $\operatorname{pot}\left(\boldsymbol{\Delta}_{2}^{0}\right)$ s-acyclic relation containing $A$. By Lemma 8.2, (a) and (b) cannot hold simultaneously. So assume that (a) does not hold. Then $A$ is not separable from $B \cap R$ by a $\operatorname{pot}\left(D_{\eta}\left(\boldsymbol{\Sigma}_{2}^{0}\right)\right)$ set. This allows us to apply Theorem 8.3

Corollary 8.5 Let $1 \leq \eta<\omega, X$ be a Polish space, and $A, B$ be disjoint analytic relations on $X$. The following are equivalent:

(1) there is $R \in \Sigma_{1}^{1}$ s-acyclic such that $A \cap R$ is not separable from $B \cap R$ by a pot $\left(D_{\eta}\left(\boldsymbol{\Sigma}_{2}^{0}\right)\right)$ set,

(2) there is $f: 2^{\omega} \rightarrow X$ injective continuous such that $\mathbb{N}_{\eta} \subseteq(f \times f)^{-1}(A)$ and $\lceil T\rceil \backslash \mathbb{N}_{\eta} \subseteq(f \times f)^{-1}(B)$. 
Proof. (1) $\Rightarrow$ (2) We apply Theorem 8.3

(2) $\Rightarrow(1)$ We can take $R:=(f \times f)[\lceil T\rceil]$.

\section{Oriented graphs}

Proof of Theorem 1.9. Theorem 1.3 provides Borel relations $\mathbb{S}_{0}, \mathbb{S}_{1}$ on $2^{\omega}$. We saw that $\mathbb{S}_{0} \cup \mathbb{S}_{1}$ is a subset of the body of a tree $T$, which does not depend on $\boldsymbol{\Gamma}$, and is contained in $N_{0} \times N_{1}$. We set $\mathbb{G}_{\boldsymbol{\Gamma}}:=\mathbb{S}_{0} \cup\left(\mathbb{S}_{1}\right)^{-1}$, so that $\mathbb{G}_{\boldsymbol{\Gamma}}$ is Borel. As $\mathbb{S}_{0} \cup \mathbb{S}_{1} \subseteq N_{0} \times N_{1}$ and $\mathbb{S}_{0}, \mathbb{S}_{1}$ are disjoint, $\mathbb{G}_{\boldsymbol{\Gamma}}$ is an oriented graph. If (a) and (b) hold, then $\mathbb{G}_{\boldsymbol{\Gamma}}$ is separable from $\mathbb{G}_{\boldsymbol{\Gamma}}^{-1}$ by a $\operatorname{pot}(\boldsymbol{\Gamma})$ set $S$. Note that $S$ also separates $\mathbb{S}_{0}=\mathbb{G}_{\boldsymbol{\Gamma}} \cap\left(N_{0} \times N_{1}\right)$ from $\mathbb{S}_{1}=\mathbb{G}_{\boldsymbol{\Gamma}}^{-1} \cap\left(N_{0} \times N_{1}\right)$, which is absurd. Thus (a) and (b) cannot hold simultaneously.

Assume now that (a) does not hold. Then there are $g, h: 2^{\omega} \rightarrow X$ continuous such that the inclusions $\mathbb{S}_{0} \subseteq(g \times h)^{-1}(G)$ and $\mathbb{S}_{1} \subseteq(g \times h)^{-1}\left(G^{-1}\right)$ hold. It remains to set $f(0 \alpha):=g(0 \alpha)$ and $f(1 \beta):=h(1 \beta)$.

Proof of Theorem 1.14. We argue as in the proof of Theorem 1.9 The things to note are the following:

- if $G$ is s-acyclic or locally countable, then $s(G)$ too,

- as noted in [Lo4], if $G$ is separable from $G^{-1}$ by a $\operatorname{pot}(\boldsymbol{\Gamma})$ set $S$, then $S^{-1} \in \operatorname{pot}(\boldsymbol{\Gamma})$ separates $G^{-1}$ from $G$, and $\neg S^{-1} \in \operatorname{pot}(\check{\boldsymbol{\Gamma}})$ separates $G$ from $G^{-1}$, so that we can restrict our attention to the classes $D_{\eta}\left(\boldsymbol{\Sigma}_{\xi}^{0}\right)$ and $\boldsymbol{\Delta}_{2}^{0}$.

- If $\boldsymbol{\Gamma}$ has rank two, then Theorem 8.3 and Corollary 7.3 provide Borel relations $\mathbb{S}_{0}, \mathbb{S}_{1}$ on $2^{\omega}$.

- If $\boldsymbol{\Gamma}=D_{\eta}\left(\boldsymbol{\Sigma}_{1}^{0}\right)$, then Corollaries 3.6 and 3.9 provide $f: 2^{\omega} \rightarrow X$ injective continuous such that one of the following holds:

(a) $\mathbb{N}_{0}^{\eta} \subseteq(f \times f)^{-1}(G)$ and $\mathbb{N}_{1}^{\eta} \subseteq(f \times f)^{-1}\left(G^{-1}\right)$,

(b) $\mathbb{B}_{0}^{\eta} \subseteq(f \times f)^{-1}(G)$ and $\mathbb{B}_{1}^{\eta} \subseteq(f \times f)^{-1}\left(G^{-1}\right)$.

The case (a) cannot happen since $G^{-1}$ is irreflexive.

Proof of Theorem 1.15. Note first that $\mathbb{S}_{0}^{\eta} \cup\left(\mathbb{S}_{1}^{\eta}\right)^{-1}, \mathbb{C}_{0}^{\eta} \cup\left(\mathbb{C}_{1}^{\eta}\right)^{-1}, \mathbb{B}_{0}^{\eta} \cup\left(\mathbb{B}_{1}^{\eta}\right)^{-1}$ and $\mathbb{B}_{1}^{\eta} \cup\left(\mathbb{B}_{0}^{\eta}\right)^{-1}$ are Borel oriented graphs with locally countable closure. As in the proof of Theorem 1.9 , $\mathbb{G}$ is not separable from $\mathbb{G}^{-1}$ by a pot $\left(\Delta\left(D_{\eta}\left(\boldsymbol{\Sigma}_{1}^{0}\right)\right)\right)$ set if $\mathbb{G} \in\left\{\mathbb{C}_{0}^{\eta} \cup\left(\mathbb{C}_{1}^{\eta}\right)^{-1}, \mathbb{B}_{0}^{\eta} \cup\left(\mathbb{B}_{1}^{\eta}\right)^{-1}, \mathbb{B}_{1}^{\eta} \cup\left(\mathbb{B}_{0}^{\eta}\right)^{-1}\right\}$. By Lemma 3.1, $\mathbb{S}_{0}^{\eta} \cup\left(\mathbb{S}_{1}^{\eta}\right)^{-1}$ is not separable from $\left(\mathbb{S}_{0}^{\eta}\right)^{-1} \cup \mathbb{S}_{1}^{\eta}$ by a $\operatorname{pot}\left(\Delta\left(D_{\eta}\left(\boldsymbol{\Sigma}_{1}^{0}\right)\right)\right)$ set.

- Assume now that (a) does not hold. Corollaries 4.5 and 4.7 provide

$$
(\mathbb{A}, \mathbb{B}) \in\left\{\left(\mathbb{N}_{1}^{\eta}, \mathbb{N}_{0}^{\eta}\right),\left(\mathbb{B}_{1}^{\eta}, \mathbb{B}_{0}^{\eta}\right),\left(\mathbb{N}_{0}^{\eta}, \mathbb{N}_{1}^{\eta}\right),\left(\mathbb{B}_{0}^{\eta}, \mathbb{B}_{1}^{\eta}\right),\left(\mathbb{S}_{0}^{\eta}, \mathbb{S}_{1}^{\eta}\right),\left(\mathbb{C}_{0}^{\eta}, \mathbb{C}_{1}^{\eta}\right)\right\}
$$

and $f: 2^{\omega} \rightarrow X$ injective continuous such that $\mathbb{A} \subseteq(f \times f)^{-1}(G)$ and $\mathbb{B} \subseteq(f \times f)^{-1}\left(G^{-1}\right)$. 
The pair $(\mathbb{A}, \mathbb{B})$ cannot be in $\left\{\left(\mathbb{N}_{1}^{\eta}, \mathbb{N}_{0}^{\eta}\right),\left(\mathbb{N}_{0}^{\eta}, \mathbb{N}_{1}^{\eta}\right)\right\}$ since $G$ and $G^{-1}$ are irreflexive. It is enough to show the existence of $f: 2^{\omega} \rightarrow 2^{\omega}$ injective continuous such that $\mathbb{B}_{0}^{\eta} \cup\left(\mathbb{B}_{1}^{\eta}\right)^{-1} \subseteq(f \times f)^{-1}\left(\mathbb{B}_{1}^{\eta} \cup\left(\mathbb{B}_{0}^{\eta}\right)^{-1}\right)$ to see that (b) holds.

- We use the notation of the proof of Proposition 4.4 Let us show that

$$
F_{\theta}^{\text {parity }(\eta)}:=F_{\theta, 1}^{\text {parity }(\eta)} \subseteq C_{\theta}
$$

if $\theta<\eta$ (where $A_{\varepsilon}=\mathbb{N}_{\varepsilon}^{\eta}$ and the closures refer to $\tau_{1}$ ). We argue by induction on $\theta$. Note first that

$$
F_{0}^{\text {parity }(\eta)}=\overline{\mathbb{N}_{\operatorname{parity}(\eta)}^{\eta}}=\overline{\bigcup_{\operatorname{parity}(\varphi(s))=0} \operatorname{Gr}\left(f_{s}\right)} \subseteq \overline{C_{0}}=C_{0},
$$

by the proof of Proposition 4.4 Then, inductively,

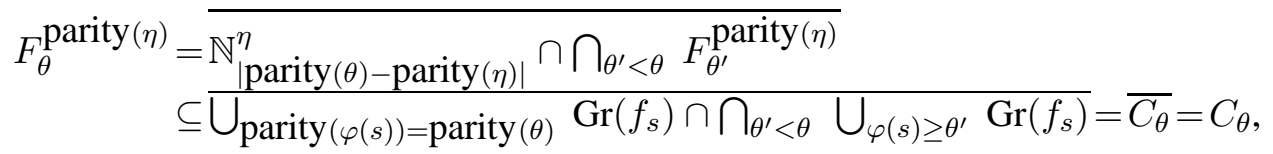

by the proof of Proposition 4.4

- From this we deduce that $\mathbb{N}_{0}^{\eta} \cap \bigcap_{\theta<\eta} F_{\theta}^{\text {parity }(\eta)}$ is contained in

$$
\left(\bigcup_{\operatorname{parity}(\varphi(s))=\operatorname{parity}(\eta)} \operatorname{Gr}\left(f_{s}\right)\right) \cap \bigcap_{\theta<\eta} C_{\theta} \subseteq \operatorname{Gr}\left(f_{\emptyset}\right)=\Delta\left(2^{\omega}\right) .
$$

As $\mathbb{N}_{0}^{\eta} \cup \mathbb{N}_{1}^{\eta}$ is locally countable and $\mathbb{N}_{0}^{\eta} \cap \bigcap_{\theta<\eta} F_{\theta}^{\text {parity }(\eta)} \subseteq \Delta\left(2^{\omega}\right)$, the proof of Theorem 3.3 gives $h: 2^{\omega} \rightarrow 2^{\omega}$ injective continuous such that $\mathbb{N}_{0}^{\eta} \subseteq(h \times h)^{-1}\left(\left(\mathbb{N}_{0}^{\eta}\right)^{-1}\right)$ and $\mathbb{N}_{1}^{\eta} \subseteq(h \times h)^{-1}\left(\left(\mathbb{N}_{1}^{\eta}\right)^{-1}\right)$ (we are in the case 2 of this proof). The map $f: \varepsilon \alpha \mapsto(1-\varepsilon) h(\alpha)$ is as desired.

- As $\Delta\left(2^{\omega}\right)$ is contained in the closure of $\mathbb{S}_{0}^{\eta} \cup\left(\mathbb{S}_{1}^{\eta}\right)^{-1}$, this last relation is not below the two others.

- Assume, towards a contradiction, that $\mathbb{B}_{0}^{\eta} \cup\left(\mathbb{B}_{1}^{\eta}\right)^{-1}$ is below $\mathbb{S}_{0}^{\eta} \cup\left(\mathbb{S}_{1}^{\eta}\right)^{-1}$. This gives $s \in 2^{<\omega}$ and $\varepsilon \in 2$ such that $\left(N_{0 s}, N_{1 s}, \mathbb{B}_{0}^{\eta} \cap\left(N_{0 s} \times N_{1 s}\right), \mathbb{B}_{1}^{\eta} \cap\left(N_{0 s} \times N_{1 s}\right)\right) \sqsubseteq\left(2^{\omega}, 2^{\omega},\left(\mathbb{S}_{\varepsilon}^{\eta}\right)^{1-2 \varepsilon},\left(\mathbb{S}_{1-\varepsilon}^{\eta}\right)^{1-2 \varepsilon}\right)$. By Lemma 3.1, $\mathbb{N}_{0}^{\eta} \cap N_{s}^{2}$ is not separable from $\mathbb{N}_{1}^{\eta} \cap N_{s}^{2}$ by a $\operatorname{pot}\left(D_{\eta}\left(\Sigma_{1}^{0}\right)\right)$ set. As $\mathbb{N}_{0}^{\eta} \cup \mathbb{N}_{1}^{\eta}$ is locally countable and $\mathbb{N}_{0}^{\eta} \cap \bigcap_{\theta<\eta} F_{\theta}^{\text {parity }(\eta)} \subseteq \Delta\left(2^{\omega}\right)$, the proof of Theorem 3.3 gives $h: 2^{\omega} \rightarrow N_{s}$ injective continuous such that $\mathbb{N}_{\epsilon}^{\eta} \subseteq(h \times h)^{-1}\left(\mathbb{N}_{\epsilon}^{\eta} \cap N_{s}^{2}\right)$ for each $\epsilon \in 2$ (we are in the case 2 of this proof). This implies that $\left(2^{\omega}, 2^{\omega}, \mathbb{B}_{0}^{\eta}, \mathbb{B}_{1}^{\eta}\right) \sqsubseteq\left(N_{0 s}, N_{1 s}, \mathbb{B}_{0}^{\eta} \cap\left(N_{0 s} \times N_{1 s}\right), \mathbb{B}_{1}^{\eta} \cap\left(N_{0 s} \times N_{1 s}\right)\right)$ and

$$
\left(2^{\omega}, 2^{\omega}, \mathbb{B}_{0}^{\eta}, \mathbb{B}_{1}^{\eta}\right) \sqsubseteq\left(2^{\omega}, 2^{\omega},\left(\mathbb{S}_{\varepsilon}^{\eta}\right)^{1-2 \varepsilon},\left(\mathbb{S}_{1-\varepsilon}^{\eta}\right)^{1-2 \varepsilon}\right) .
$$

By Corollary 3.9, $\left(2^{\omega}, 2^{\omega}, \mathbb{N}_{0}^{\eta}, \mathbb{N}_{1}^{\eta}\right) \sqsubseteq\left(2^{\omega}, 2^{\omega}, \mathbb{B}_{0}^{\eta}, \mathbb{B}_{1}^{\eta}\right)$, so that

$$
\left(2^{\omega}, 2^{\omega}, \mathbb{N}_{0}^{\eta}, \mathbb{N}_{1}^{\eta}\right) \sqsubseteq\left(2^{\omega}, 2^{\omega},\left(\mathbb{S}_{\varepsilon}^{\eta}\right)^{1-2 \varepsilon},\left(\mathbb{S}_{1-\varepsilon}^{\eta}\right)^{1-2 \varepsilon}\right) .
$$

But this contradicts the proof of Proposition 4.4 
- We will show that $\left(2^{\omega}, 2^{\omega}, \mathbb{C}_{0}^{\eta}, \mathbb{C}_{1}^{\eta}\right) \sqsubseteq\left(2^{\omega}, 2^{\omega}, \mathbb{S}_{0}^{\eta}, \mathbb{S}_{1}^{\eta}\right)$. Using the proof of the previous point, this will show that $\mathbb{B}_{0}^{\eta} \cup\left(\mathbb{B}_{1}^{\eta}\right)^{-1}$ is not below $\mathbb{C}_{0}^{\eta} \cup\left(\mathbb{C}_{1}^{\eta}\right)^{-1}$.

We use the notation of the proof of Proposition 4.4 Let us show that $G_{\theta}:=G_{\theta, 1} \subseteq C_{\theta}$ if $1 \leq \theta \leq \eta$ (where $A_{\varepsilon}=\mathbb{S}_{\varepsilon}^{\eta}$ and the closures refer to $\tau_{1}$ ). We argue by induction on $\theta$. Note first that

$$
G_{1}=\overline{\mathbb{S}_{0}^{\eta}} \cap \overline{\mathbb{S}_{1}^{\eta}}=\overline{U_{0}^{0}} \cap \overline{U_{0}^{1}}=C_{1}^{0} \cup C_{1}^{1}=C_{1}
$$

by the proof of Proposition 4.4. Then, inductively,

$$
G_{\theta+1}=\overline{\mathbb{S}_{0}^{\eta} \cap G_{\theta}} \cap \overline{\mathbb{S}_{1}^{\eta} \cap G_{\theta}} \subseteq \overline{U_{0}^{0} \cap C_{\theta}} \cap \overline{U_{0}^{1} \cap C_{\theta}} \subseteq C_{\theta+1}
$$

and $G_{\lambda}=\bigcap_{\theta<\lambda} G_{\theta} \subseteq \bigcap_{\theta<\lambda} C_{\theta}=C_{\lambda}$ if $\lambda$ is limit.

From this we deduce that $G_{\eta} \subseteq C_{\eta}=\operatorname{Gr}\left(f_{\emptyset}\right)=\Delta\left(2^{\omega}\right)$. As $\mathbb{S}_{0}^{\eta} \cup \mathbb{S}_{1}^{\eta}$ is locally countable and $G_{\eta} \subseteq \Delta\left(2^{\omega}\right)$, the proof of Theorem 4.3 gives $h: 2^{\omega} \rightarrow N_{s}$ injective continuous such that the inclusion $\mathbb{S}_{\epsilon}^{\eta} \subseteq(h \times h)^{-1}\left(\mathbb{S}_{\epsilon}^{\eta} \cap N_{0}^{2}\right)$ holds for each $\epsilon \in 2$ (we are in the case 2 of this proof). The maps defined by $f(0 \alpha):=h(\alpha), f(1 \alpha):=1 \alpha, g(1 \beta):=h(\beta)$ and $g(0 \beta):=1 \beta$, are as desired.

- Assume, towards a contradiction, that $\mathbb{C}_{0}^{\eta} \cup\left(\mathbb{C}_{1}^{\eta}\right)^{-1}$ is below $\mathbb{S}_{0}^{\eta} \cup\left(\mathbb{S}_{1}^{\eta}\right)^{-1}$, with witness $f$. This gives $s \in 2^{<\omega} \backslash\{\emptyset\}$ and $\varepsilon \in 2$ such that $\mathbb{C}_{\epsilon}^{\eta} \cap\left(N_{0 s} \times N_{1 s}\right) \subseteq(f \times f)^{-1}\left(\left(\mathbb{S}_{|\epsilon-\varepsilon|}^{\eta}\right)^{1-2 \varepsilon}\right)$ for each $\epsilon \in 2$. As in the previous point, there is $h: 2^{\omega} \rightarrow N_{s}$ injective continuous such that

$$
\mathbb{S}_{\epsilon}^{\eta} \subseteq(h \times h)^{-1}\left(\mathbb{S}_{\epsilon}^{\eta} \cap N_{s}^{2}\right)
$$

for each $\epsilon \in 2$. This implies that if we set $k(\epsilon \alpha):=\epsilon h(\alpha)$ and $l:=f \circ k$, then

$$
\mathbb{C}_{\epsilon}^{\eta} \subseteq(k \times k)^{-1}\left(\mathbb{C}_{\epsilon}^{\eta} \cap\left(N_{0 s} \times N_{1 s}\right)\right)
$$

and $\mathbb{C}_{\epsilon}^{\eta} \subseteq(l \times l)^{-1}\left(\left(\mathbb{S}_{|\epsilon-\varepsilon|}^{\eta}\right)^{1-2 \varepsilon}\right)$. As in the proof of Proposition 4.4, we see that the image of

$$
\left\{(0 \alpha, 1 \alpha) \mid \alpha \in 2^{\omega}\right\}
$$

by $l \times l$ is contained in the diagonal of $2^{\omega}$, which is not possible by injectivity of $l$.

- Assume that $\eta$ is a successor ordinal. The previous points show that if $\mathbb{C}_{0}^{\eta} \cup\left(\mathbb{C}_{1}^{\eta}\right)^{-1}$ is below $\mathbb{B}_{0}^{\eta} \cup\left(\mathbb{B}_{1}^{\eta}\right)^{-1}$, then $\left(2^{\omega}, 2^{\omega}, \mathbb{C}_{0}^{\eta}, \mathbb{C}_{1}^{\eta}\right) \sqsubseteq\left(2^{\omega}, 2^{\omega},\left(\mathbb{B}_{\varepsilon}^{\eta}\right)^{1-2 \varepsilon},\left(\mathbb{B}_{1-\varepsilon}^{\eta}\right)^{1-2 \varepsilon}\right)$ for some $\varepsilon \in 2$. We saw that there is $h: 2^{\omega} \rightarrow N_{0}$ injective continuous such that $\mathbb{N}_{\epsilon}^{\eta} \subseteq(h \times h)^{-1}\left(\mathbb{N}_{\epsilon}^{\eta} \cap N_{0}^{2}\right)$ for each $\epsilon \in 2$. The maps defined by $f(0 \alpha):=h(\alpha), f(1 \alpha):=1 \alpha, g(1 \beta):=h(\beta)$ and $g(0 \beta):=1 \beta$ are witnesses for the fact that $\left(2^{\omega}, 2^{\omega}, \mathbb{B}_{0}^{\eta}, \mathbb{B}_{1}^{\eta}\right) \sqsubseteq\left(2^{\omega}, 2^{\omega}, \mathbb{N}_{0}^{\eta}, \mathbb{N}_{1}^{\eta}\right)$, so that $\left(2^{\omega}, 2^{\omega}, \mathbb{C}_{0}^{\eta}, \mathbb{C}_{1}^{\eta}\right) \sqsubseteq\left(2^{\omega}, 2^{\omega},\left(\mathbb{N}_{\varepsilon}^{\eta}\right)^{1-2 \varepsilon},\left(\mathbb{N}_{1-\varepsilon}^{\eta}\right)^{1-2 \varepsilon}\right)$. The maps $\alpha \mapsto 0 \alpha$ and $\beta \mapsto 1 \beta$ are witnesses for the fact that $\left(2^{\omega}, 2^{\omega}, \mathbb{S}_{0}^{\eta}, \mathbb{S}_{1}^{\eta}\right) \sqsubseteq\left(2^{\omega}, 2^{\omega}, \mathbb{C}_{0}^{\eta}, \mathbb{C}_{1}^{\eta}\right)$. Thus $\left(2^{\omega}, 2^{\omega}, \mathbb{S}_{0}^{\eta}, \mathbb{S}_{1}^{\eta}\right) \sqsubseteq\left(2^{\omega}, 2^{\omega},\left(\mathbb{N}_{\varepsilon}^{\eta}\right)^{1-2 \varepsilon},\left(\mathbb{N}_{1-\varepsilon}^{\eta}\right)^{1-2 \varepsilon}\right)$, which contradicts the proof of Proposition 4.4

- Assume that $\eta$ is a limit ordinal. Let us show that $\mathbb{C}_{0}^{\eta} \cup\left(\mathbb{C}_{1}^{\eta}\right)^{-1}$ is below $\mathbb{B}_{0}^{\eta} \cup\left(\mathbb{B}_{1}^{\eta}\right)^{-1}$. The proof of Proposition 4.4 provides $h: 2^{\omega} \rightarrow 2^{\omega}$ injective continuous such that $\mathbb{S}_{\varepsilon}^{\eta} \subseteq(h \times h)^{-1}\left(\mathbb{N}_{\varepsilon}^{\eta}\right)$ for each $\varepsilon \in 2$. It remains to set $f(\varepsilon \alpha):=\varepsilon h(\alpha)$. 


\section{Negative results}

- By Theorem 15 in [L4], we cannot completely remove the assumption that $A$ is s-acyclic or locally countable in Corollary 6.4 We can wonder whether there is an antichain basis if this assumption is removed (for this class $\Pi_{2}^{0}$ or any other one appearing in this section). This also shows that we cannot simply assume the disjointness of the analytic sets $A, B$ in Theorem 6.3 and Corollaries 6.5 6.7

- We can use the proof of the previous fact to get a negative result for the class $\Delta_{2}^{0}$.

Theorem 10.1 There is no tuple $(\mathbb{X}, \mathbb{Y}, \mathbb{A}, \mathbb{B})$, where $\mathbb{X}, \mathbb{Y}$ are Polish and $\mathbb{A}, \mathbb{B}$ are disjoint analytic subsets of $\mathbb{X} \times \mathbb{Y}$, such that for any tuple $(\mathcal{X}, \mathcal{Y}, \mathcal{A}, \mathcal{B})$ of this type, exactly one of the following holds:

(a) $\mathcal{A}$ is separable from $\mathcal{B}$ by a pot $\left(\boldsymbol{\Delta}_{2}^{0}\right)$ set,

(b) $(\mathbb{X}, \mathbb{Y}, \mathbb{A}, \mathbb{B}) \sqsubseteq(\mathcal{X}, \mathcal{Y}, \mathcal{A}, \mathcal{B})$.

Proof. We argue by contradiction. By Lemma 7.1 we get $(\mathbb{X}, \mathbb{Y}, \mathbb{A}, \mathbb{B}) \sqsubseteq\left(2^{\omega}, 2^{\omega},\lceil T\rceil \cap \mathbb{E}_{0}^{0},\lceil T\rceil \cap \mathbb{E}_{0}^{1}\right)$. This shows that $\mathbb{A}, \mathbb{B}$ are locally countable. As (a) and (b) cannot hold simultaneously, $\mathbb{A}$ is not separable from $\mathbb{B}$ by $\operatorname{apot}\left(\boldsymbol{\Delta}_{2}^{0}\right)$ set. By Corollary 7.4 we get

$$
\left(2^{\omega}, 2^{\omega},\lceil T\rceil \cap \mathbb{E}_{0}^{0},\lceil T\rceil \cap \mathbb{E}_{0}^{1}\right) \sqsubseteq(\mathbb{X}, \mathbb{Y}, \mathbb{A}, \mathbb{B}),
$$

so that we may assume that $(\mathbb{X}, \mathbb{Y}, \mathbb{A}, \mathbb{B})=\left(2^{\omega}, 2^{\omega},\lceil T\rceil \cap \mathbb{E}_{0}^{0},\lceil T\rceil \cap \mathbb{E}_{0}^{1}\right)$.

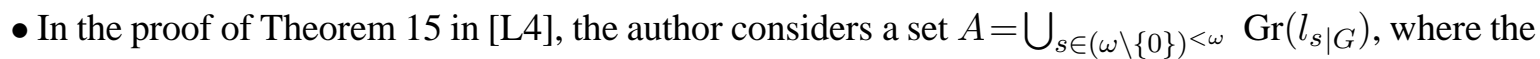
$l_{s}$ 's are partial continuous open maps from $2^{\omega}$ into itself with dense open domain, and $G$ is the intersection of their domain. Moreover, the $l_{s}$ 's have the properties that $l_{s}(x) \neq l_{t}(x)$ if $t \neq s$, and $l_{s}(x)$ is the limit of $\left(l_{s k}(x)\right)_{k \in \omega}$, for each $x \in G$. We set, for $\varepsilon \in 2, A_{\varepsilon}:=\bigcup_{s \in(\omega \backslash\{0\})<\omega,|s| \equiv \varepsilon(\bmod 2)} \operatorname{Gr}\left(l_{s \mid G}\right)$, so that $A_{0}$ and $A_{1}$ are disjoint Borel sets.

Let us check that $A_{0}$ is not separable from $A_{1}$ by $\operatorname{apot}\left(\boldsymbol{\Delta}_{2}^{0}\right)$ set. We argue by contradiction, which gives $D \in \operatorname{pot}\left(\boldsymbol{\Delta}_{2}^{0}\right)$ and a dense $G_{\delta}$ subset $H$ of $2^{\omega}$ such that $D \cap H^{2} \in \boldsymbol{\Delta}_{2}^{0}\left(H^{2}\right)$. We may assume that $H \subseteq G$. Note that $H \cap \bigcap_{s \in(\omega \backslash\{0\})^{<\omega}} l_{s}^{-1}(H)$ is a dense $G_{\delta}$ subset of $2^{\omega}$, and thus contains a point $x$. The vertical section $A_{x}$ is contained in $H$. In particular, the disjoint sections $\left(A_{0}\right)_{x}$ and $\left(A_{1}\right)_{x}$ are separable by a $\Delta_{2}^{0}$ subset $\mathcal{D}$ of the Polish space $H$. It remains to note that $\mathcal{D} \cap{\overline{A_{x}}}^{H}$ is a dense and co-dense $\Delta_{2}^{0}$ subset of ${\overline{A_{x}}}^{H}$, which contradicts Baire's theorem.

This gives $u: N_{0} \rightarrow 2^{\omega}$ and $v: N_{1} \rightarrow 2^{\omega}$ with $\lceil T\rceil \cap \mathbb{E}_{0}^{\varepsilon} \subseteq(u \times v)^{-1}\left(A_{\varepsilon}\right)$.

- We set $B_{1}:=\lceil T\rceil \cap\left(\mathbb{E}_{0}^{0} \cup \mathbb{E}_{0}^{1}\right)$. Note that $B_{1} \notin \operatorname{pot}\left(G_{\delta}\right)$, since otherwise $\lceil T\rceil \cap \mathbb{E}_{0}^{0}$ and $\lceil T\rceil \cap \mathbb{E}_{0}^{1}$ are two disjoint $\operatorname{pot}\left(G_{\delta}\right)$ sets, and thus $\operatorname{pot}\left(\boldsymbol{\Delta}_{2}^{0}\right)$-separable. Then we can follow the proof of Theorem 15 in [L4]. This proof gives $U: F \rightarrow G$ and $V: F \rightarrow 2^{\omega}$ injective continuous satisfying the inclusion $\bigcup_{n \in \omega} \operatorname{Gr}\left(f_{n}\right) \subseteq(U \times V)^{-1}(A)$.

The only thing to check is that there is $(c, d)$ in $\bigcup_{n \in \omega} \omega^{n} \times \omega^{n+1}$ and a nonempty open subset $R$ of $D_{f_{c, d}}$ such that $\left(U(x), V\left(f_{c, d}(x)\right)\right) \notin \operatorname{Gr}\left(l_{\emptyset}\right)$ for each $x \in R$. We argue by contradiction, which gives a dense $G_{\delta}$ subset $K$ of $F$ such that $\bigcup_{n \in \omega} \operatorname{Gr}\left(f_{n \mid K}\right) \subseteq\left(U_{\mid K} \times V\right)^{-1}\left(\operatorname{Gr}\left(l_{\emptyset \mid G}\right)\right)$. As $\left(U_{\mid K} \times V\right)^{-1}\left(\operatorname{Gr}\left(l_{\emptyset \mid G}\right)\right)$ is the graph of a partial Borel map, $\bigcup_{n \in \omega} \operatorname{Gr}\left(f_{n \mid K}\right)$ too. Therefore $\bigcup_{n \in \omega} \operatorname{Gr}\left(f_{n \mid K}\right) \in \operatorname{pot}\left(\boldsymbol{\Pi}_{1}^{0}\right) \backslash \operatorname{pot}\left(G_{\delta}\right)$, which is absurd. 
This shows that we cannot completely remove the assumption that $A \cup B$ is s-acyclic or locally countable in Corollary 7.3 This also shows that we cannot simply assume the disjointness of the analytic sets $A, B$ in Theorem 7.2 and Corollary 7.4

- By Theorem 2.16 in [L3], we cannot completely remove the assumption that $A \cup B$ is s-acyclic or locally countable in Corollary 3.10 . This also shows that we cannot simply assume disjointness in Theorem 3.3 and Corollary 3.11

We saw that there is a version of Corollary 6.7 for $\boldsymbol{\Gamma}=\Sigma_{1}^{0}$, where we replace the class $F_{\sigma}$ with the class of open sets. We cannot replace the class $F_{\sigma}$ with the class of closed sets.

Proposition 10.2 There is no triple $(\mathbb{X}, \mathbb{A}, \mathbb{B})$, where $\mathbb{X}$ is Polish and $\mathbb{A}, \mathbb{B}$ are disjoint analytic relations on $\mathbb{X}$ such that $\mathbb{A}$ is contained in a potentially closed s-acyclic or locally countable relation such that, for each triple $(\mathcal{X}, \mathcal{A}, \mathcal{B})$ of the same type, exactly one of the following holds:

(a) the set $\mathcal{A}$ is separable from $\mathcal{B}$ by a pot $\left(\Sigma_{1}^{0}\right)$ set,

(b) $(\mathbb{X}, \mathbb{X}, \mathbb{A}, \mathbb{B}) \sqsubseteq(\mathcal{X}, \mathcal{X}, \mathcal{A}, \mathcal{B})$.

Proof. We argue by contradiction, which gives a triple. Note that $\mathbb{A}$ is not separable from $\mathbb{B}$ by a $\operatorname{pot}\left(\boldsymbol{\Sigma}_{1}^{0}\right)$ set. Theorem 9 in [L5] gives $F, G: 2^{\omega} \rightarrow \mathbb{X}$ continuous such that $\Delta\left(2^{\omega}\right) \subseteq(F \times G)^{-1}(\mathbb{A})$ and $\mathbb{G}_{0} \subseteq(F \times G)^{-1}(\mathbb{B})$. We set $\mathbb{A}^{\prime}:=(F \times G)\left[\Delta\left(2^{\omega}\right)\right], \mathbb{B}^{\prime}:=(F \times G)\left[\mathbb{G}_{0}\right]$ and $\mathbb{C}^{\prime}:=(F \times G)\left[\overline{\mathbb{G}_{0}}\right]$. Note that $\mathbb{A}^{\prime}, \mathbb{C}^{\prime}$ are compact and $\mathbb{C}^{\prime}$ is the locally countable disjoint union of $\mathbb{A}^{\prime}$ and $\mathbb{B}^{\prime}$. In particular, $\mathbb{B}^{\prime}$ is $D_{2}\left(\Sigma_{1}^{0}\right), \mathbb{A}^{\prime} \subseteq \mathbb{A}, \mathbb{B}^{\prime} \subseteq \mathbb{B}$, and $\mathbb{A}^{\prime}$ is not separable from $\mathbb{B}^{\prime}$ by a $\operatorname{pot}\left(\boldsymbol{\Sigma}_{1}^{0}\right)$ set. So we may assume that $\mathbb{A}, \mathbb{B}$ are Borel with locally countable union which is the closure of $\mathbb{B}$. Corollary 3.10 gives $f^{\prime}, g^{\prime}: 2^{\omega} \rightarrow \mathbb{X}$ injective continuous such that $\mathbb{G}_{0}=\overline{\mathbb{G}_{0}} \cap\left(f^{\prime} \times g^{\prime}\right)^{-1}(\mathbb{B})$. In particular,

$$
\Delta\left(2^{\omega}\right) \subseteq\left(f^{\prime} \times g^{\prime}\right)^{-1}(\overline{\mathbb{B}} \backslash \mathbb{B})=\left(f^{\prime} \times g^{\prime}\right)^{-1}(\mathbb{A}) .
$$

This means that we may assume that $\mathbb{X}=2^{\omega}, \mathbb{A}=\Delta\left(2^{\omega}\right)$ and $\mathbb{B}=\mathbb{G}_{0}$.

The proof of Theorem 10 in [L5] provides a Borel graph $\mathcal{B}$ on $X:=2^{\omega}$ with no Borel countable coloring such that any locally countable Borel digraph contained in $\mathcal{B}$ has a Borel countable coloring. Consider the closed symmetric acyclic locally countable relation $\mathcal{A}:=\Delta\left(2^{\omega}\right)$. As there is no Borel countable coloring of $\mathcal{B}, \mathcal{A}$ is not separable from $\mathcal{B}$ by a $\operatorname{pot}\left(\boldsymbol{\Sigma}_{1}^{0}\right)$ set. If $f, g$ exist, then $f=g$ since $\mathbb{A}$ is contained in $(f \times g)^{-1}(\mathcal{A})$. This implies that $f$ is a homomorphism from $\mathbb{G}_{0}$ into $\mathcal{B}$. The digraph $(f \times f)\left[\mathbb{G}_{0}\right]$ is locally countable and Borel since $f$ is injective. Thus it has a Borel countable coloring, and $\mathbb{G}_{0}$ too, which is absurd.

For oriented graphs, we cannot completely remove the assumption that $G$ is s-acyclic or locally countable in Theorem 1.14 Let us check it for $\Gamma=\Delta_{2}^{0}$.

Proposition 10.3 There is no tuple $(\mathbb{X}, \mathbb{G})$, where $\mathbb{X}$ is Polish and $\mathbb{G}$ is an analytic oriented graph on $\mathbb{X}$, such that for any tuple $(\mathcal{X}, \mathcal{G})$ of this type, exactly one of the following holds:

(a) the set $\mathcal{G}$ is separable from $\mathcal{G}^{-1}$ by a pot $\left(\boldsymbol{\Delta}_{2}^{0}\right)$ set,

(b) there is $f: 2^{\omega} \rightarrow X$ injective continuous such that $\mathbb{G} \subseteq(f \times f)^{-1}(\mathcal{G})$. 
Proof. We use the notation of the proof of Theorem 10.1 and argue by contradiction. Recall the analytic s-acyclic oriented graph $\mathcal{G}_{\boldsymbol{\Delta}_{2}^{0}}=\left(\lceil T\rceil \cap \mathbb{E}_{0}^{0}\right) \cup\left(\lceil T\rceil \cap \mathbb{E}_{0}^{1}\right)^{-1}$ considered in the proof of Theorem 1.14 Note that there is $f_{0}: \mathbb{X} \rightarrow 2^{\omega}$ injective continuous such that $\mathbb{G} \subseteq\left(f_{0} \times f_{0}\right)^{-1}\left(\mathcal{G}_{\boldsymbol{\Delta}_{2}^{0}}\right)$. In particular, $\mathbb{G}$ is s-acyclic and Theorem 1.14 applies. This shows that we may assume that $(\mathbb{X}, \mathbb{G})=\left(2^{\omega}, \mathcal{G}_{\boldsymbol{\Delta}_{2}^{0}}\right)$.

If $R$ is a relation on $2^{\omega}$, then we set $G_{R}:=\{(0 \alpha, 1 \beta) \mid(\alpha, \beta) \in R\}$. As $A_{0}$ is not separable from $A_{1}$ by a $\operatorname{pot}\left(\boldsymbol{\Delta}_{2}^{0}\right)$ set, $G_{A_{0}}$ is not separable from $G_{A_{1}}$ by a $\operatorname{pot}\left(\boldsymbol{\Delta}_{2}^{0}\right)$ set. As $G_{A_{0}} \cup G_{A_{1}} \subseteq N_{0} \times N_{1}$ and $G_{A_{0}}, G_{A_{1}}$ are disjoint, $\mathbb{H}:=G_{A_{0}} \cup\left(G_{A_{1}}\right)^{-1}$ is a Borel oriented graph, and $\mathbb{H}$ is not separable from $\mathbb{H}^{-1}$ by a $\operatorname{pot}\left(\Delta_{2}^{0}\right)$ set, as in the proof of Theorem 1.9 If $f: 2^{\omega} \rightarrow 2^{\omega}$ is injective continuous and $\left(\lceil T\rceil \cap \mathbb{E}_{0}^{0}\right) \cup\left(\lceil T\rceil \cap \mathbb{E}_{0}^{1}\right)^{-1} \subseteq \mathbb{H}$, then on a nonempty clopen set $S:=N_{s_{q}} \times N_{t_{q}}$, the first coordinate is either preserved, or changed.

As in the proof of Lemma 7.1, we see that $\lceil T\rceil \cap \mathbb{E}_{0}^{0} \cap S$ is not separable from $\lceil T\rceil \cap \mathbb{E}_{0}^{1} \cap S$ by a $\operatorname{pot}\left(\boldsymbol{\Delta}_{2}^{0}\right)$ set. By Corollary 7.3 , there is $f: 2^{\omega} \rightarrow 2^{\omega}$ injective continuous such that

$$
\lceil T\rceil \cap \mathbb{E}_{0}^{\varepsilon} \subseteq(f \times f)^{-1}\left(\lceil T\rceil \cap \mathbb{E}_{0}^{\varepsilon} \cap S\right)
$$

for each $\varepsilon \in 2$. This proves the existence of $g: 2^{\omega} \rightarrow 2^{\omega}$ injective continuous such that

$$
\lceil T\rceil \cap\left(\mathbb{E}_{0}^{0} \cup \mathbb{E}_{0}^{1}\right) \subseteq(g \times g)^{-1}\left(G_{A}\right) .
$$

This gives $u: N_{0} \rightarrow 2^{\omega}$ and $v: N_{1} \rightarrow 2^{\omega}$ injective continuous such that $\lceil T\rceil \cap\left(\mathbb{E}_{0}^{0} \cup \mathbb{E}_{0}^{1}\right) \subseteq(u \times v)^{-1}(A)$ since the maps $\varepsilon \alpha \mapsto \alpha$ are injective. But we saw that this is not possible in the proof of Theorem 10.1

Question. Are there versions of our results for the classes $D_{\eta}\left(\boldsymbol{\Sigma}_{2}^{0}\right), \check{D}_{\eta}\left(\boldsymbol{\Sigma}_{2}^{0}\right)$ (when $\omega \leq \eta<\omega_{1}$ ) and $\Delta\left(D_{\eta}\left(\boldsymbol{\Sigma}_{2}^{0}\right)\right)$ (when $\left.2 \leq \eta<\omega_{1}\right)$ ? 


\section{References}

[D-SR] G. Debs and J. Saint Raymond, Borel liftings of Borel sets: some decidable and undecidable statements, Mem. Amer. Math. Soc. 187, 876 (2007)

[K] A. S. Kechris, Classical descriptive set theory, Springer-Verlag, 1995

[K-S-T] A. S. Kechris, S. Solecki and S. Todorčević, Borel chromatic numbers, Adv. Math. 141 (1999), 1-44

[L1] D. Lecomte, Classes de Wadge potentielles et théorèmes d'uniformisation partielle, Fund. Math. 143 (1993), 231-258

[L2] D. Lecomte, Uniformisations partielles et critères à la Hurewicz dans le plan, Trans. Amer. Math. Soc. 347, 11 (1995), 4433-4460

[L3] D. Lecomte, Tests à la Hurewicz dans le plan, Fund. Math. 156 (1998), 131-165

[L4] D. Lecomte, Complexité des boréliens à coupes dénombrables, Fund. Math. 165 (2000), 139174

[L5] D. Lecomte, On minimal non potentially closed subsets of the plane, Topology Appl. 154, 1 (2007), 241-262

[L6] D. Lecomte, How can we recognize potentially $\Pi_{\xi}^{0}$ subsets of the plane?, J. Math. Log. 9, 1 (2009), 39-62

[L7] D. Lecomte, A dichotomy characterizing analytic graphs of uncountable Borel chromatic number in any dimension, Trans. Amer. Math. Soc. 361 (2009), 4181-4193

[L8] D. Lecomte, Potential Wadge classes, Mem. Amer. Math. Soc., 221, 1038 (2013)

[Lo1] A. Louveau, Some results in the Wadge hierarchy of Borel sets, Cabal Sem. 79-81, Lect. Notes in Math. 1019 (1983), 28-55

[Lo2] A. Louveau, A separation theorem for $\Sigma_{1}^{1}$ sets, Trans. A. M. S. 260 (1980), 363-378

[Lo3] A. Louveau, Ensembles analytiques et boréliens dans les espaces produit, Astérisque (S. M. F.) 78 (1980)

[Lo4] A. Louveau, Some dichotomy results for analytic graphs, manuscript

[Lo-SR] A. Louveau and J. Saint Raymond, The strength of Borel Wadge determinacy, Cabal Seminar 81-85, Lecture Notes in Math. 1333 (1988), 1-30

[M] Y. N. Moschovakis, Descriptive set theory, North-Holland, 1980 